\title{
Grain boundary propagation and epitaxy on (111) surfaces of FCC substrates: A Kinetic Monte Carlo study with Lennard-Jones and Iridium potentials.
}

\author{
by \\ David John Williamson
}

A thesis

submitted to the Victoria University of Wellington in fulfilment of the requirements for the degree of Master of Science in Physics.

Victoria University of Wellington 2008 


\begin{abstract}
A Kinetic Monte Carlo (KMC) method was developed to model homoepitaxy and grain boundary propagation on a (111) surface. Barrier energies were calculated using the Nudged Elastic Band (NEB) technique. A recently reported inertial relaxation technique named FIRE (the Fast Inertial Relaxation Engine) was used to relax the NEB images. Both the LennardJones potential and a Sutton-Chen Iridium potential were used and compared.

A doubly-refined lattice mesh was developed to incorporate atoms in Face-Centred-Cubic (FCC) and Hexagonal-Close-Packed (HCP) sites as well as atoms in decorated row sites (i.e. supported by 4 atoms). A lookup table was developed to identify hops in the KMC algorithm.

The KMC results show that a small difference in energy barriers between FCC and HCP sites on the substrate can cause a substantial bias in the direction of grain boundary propagation. We also investigated the effect of the geometry of the grain boundary on its propagation, as well as the atomistic processes involved in grain boundary propagation and the merger of grain boundaries.

Our deposition simulations produced islands with loosely triangular envelopes, where FCC islands are rotated $180^{\circ}$ with respect to HCP islands. The results are similar to scanning tunneling microscopy (STM) images of Iridium deposition, although lack of computing power forced us to use a high deposition rate and this caused some differences.
\end{abstract}




\section{Acknowledgments}

Thanks are due to the following people:

Dr. Shaun Hendy, for supervision and guidance.

The Applied Maths group at IRL, in particular Dr. David Stewart for providing NEB code and other guidance and Peter Schulze for providing assistance with KMC

Prof. Thomas Michely and Dr. Carsten Busse for providing STM images

Our source of funding, The Foundation for Research, Science and Technology (FRST) Contract C08X0409 Multiscale Modelling 


\section{Contents}

1 Introduction $\quad 5$

1.1 Epitaxial Growth . . . . . . . . . . . . . . 5

1.2 Grain Boundaries . . . . . . . . . . . . . . . . 7

1.3 Atomistic simulations . . . . . . . . . . . . . 12

1.4 Outline of the following chapters . . . . . . . . . . 17

1.4.1 Chapter 2: Methodology . . . . . . . . . . 17

1.4.2 Chapter 3: Implementation ... . . . . . . . . 17

1.4.3 Chapter 4: Results and Analysis ... . . . . . . 18

1.4 .4 Chapter 5: Conclusions . . . . . . . . . 18

1.4 .5 Appendix A ................. 19

2 Methodologies $\quad 20$

2.1 Molecular Dynamics . . . . . . . . . . . . . . . . 21

2.2 Nudged Elastic Band . . . . . . . . . . . . . . . . . . . 23

2.2.1 Relaxation Methods . . . . . . . . . . . . . 29

2.3 Kinetic Monte-Carlo and transition state theory . . . . . . . . 31

3 Implementation $\quad 33$

3.1 Molecular Dynamics . . . . . . . . . . . . . . . 34

3.1.1 Integration with Velocity Verlet . . . . . . . . . . 34

3.1 .2 Potentials ................... . . 35

3.1 .3 Relaxation ................. 37

3.1.4 Nudged Elastic Band . . . . . . . . . . . . . . 38 
3.1.5 Barrier Calculations . . . . . . . . . . . 40

3.2 Kinetic Monte-Carlo . . . . . . . . . . . . . . . . 45

3.2.1 Lattice Implementation . . . . . . . . . . . . . . . . . . . . . . . . . .

3.2 .2 The look-up table . . . . . . . . . . . . . 52

3.2.3 Data structures and algorithms . . . . . . . . . . 57

3.2 .4 Other details . . . . . . . . . . . . . . 58

3.3 Adatom diffusion and determining pre-factors . . . . . . . 60

4 Results and Analysis $\quad 64$

4.1 Grain Boundary Propagation ... . . . . . . . . . . . 65

4.1 .1 Propagation of Flat Boundaries . . . . . . . . . 65

4.1 .2 Enclosed Islands . . . . . . . . . . . . 77

4.1.3 Effect of Coverage ............. . . . 79

4.2 Deposition ..................... . . 89

5 Conclusions $\quad 94$

$\begin{array}{ll}\text { A Critical hops } & 96\end{array}$ 


\section{List of Figures}

1.1 Comparison of FCC/HCP Adatoms . . . . . . . 8

1.2 Comparison of FCC/HCP Structure . . . . . . . . . 9

1.3 STM topographs of Iridium epitaxy . . . . . . . . . 11

1.4 A-gaps, B-gaps and a kink . . . . . . . . . . . . . 13

1.5 Kink-flip and pocket-fill moves. . . . . . . . . . . . . 14

2.1 Elastic band kinks . . . . . . . . . . . . . . . . . 26

3.1 LJ and IrSC two-body potential versus radius . . . . . . . 36

3.2 Atom configuration for NEB hop barrier energy calculations 39

3.3 Two examples of hops with unstable endpoints . . . . . . . 41

3.4 Initial/final neighbours versus barrier energy (LJ) . . . . 42

3.5 Initial/ final neighbours versus barrier energy (IrSC) . . . . 43

3.6 Convergence check for HCP/FCC preference in kink-flip barrier energy $(\mathrm{LJ}) \ldots \ldots \ldots \ldots . \ldots . \ldots . \ldots 47$

3.7 Convergence check for HCP/FCC preference in kink-flip

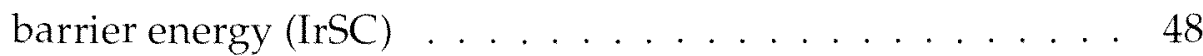

3.8 Comparison of refined lattice mesh and doubly refined lattice mesh . . . . . . . . . . . . . . . . . . . . . 49

3.9 Relevant vectors in the doubly refined mesh (FCC/HCP sites only) . . . . . . . . . . . . . . . . . 53

3.10 Relevant vectors in the doubly refined mesh (Decorated sites) 54

3.11 An invalid hop . . . . . . . . . . . . . . . 55 
3.12 KMC execution time as a function of mesh size. . . . . . . . . 59

3.13 Adatom diffusion Arrhenius plot (LJ) . . . . . . . . . . . 61

3.14 Adatom diffusion Arrhenius plot (IrSC) . . . . . . . . . . 62

4.1 Grain boundary propagation lattice initial configuration . . 66

4.2 A chevron grain boundary losing its shape . . . . . . . . . 67

4.3 FCC/HCP preference as a function of distance between grain

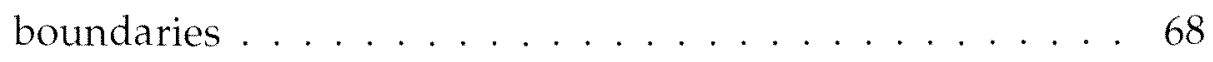

4.4 The Merger of Grain Boundaries(LJ) . . . . . . . . . . . . . 69

4.5 Arrhenius plot of grain boundary propagation (LJ) . . . . . . 71

4.6 Arrhenius plot of grain boundary propagation (IrSC) . . . . 72

4.7 Diffusion of the grain boundary versus width . . . . . . . . 73

4.8 Comparison of grain boundary merger for IrSC (lakes/bridges) and LJ (channel) . . . . . . . . . . . . . . . . . 76

4.9 Possible mechanisms for the formation of a "bridge" across the grain boundary. . . . . . . . . . . . . 78

4.10 An A-gap grain boundary heals, leaving behind a small gap that will become a channel . . . . . . . . . . . . 80

4.11 Instability of enclosed shapes . . . . . . . . . . . . . 81

4.12 Grain boundary shapes investigated . . . . . . . . . . . . 82

4.13 Channels left after healing of triangular islands . . . . . . . . 83

4.14 Two examples of a random distribution of adatoms quickly forming into islands. . . . . . . . . . . . . 84

4.15 A-gap and B-gap trimer stability . . . . . . . . . . . 86

4.16 An illustration of the instability of small A-edge islands. . . 87

4.17 String structures . . . . . . . . . . . . . . . . . 88

4.18 The dissociation of a pentamer island into decorated atoms . 90

4.19 Deposition upon the (111) plane of a periodic slab (KMC). . 91

4.20 STM images of deposition on Iridium . . . . . . . . . . . 92

4.21 Island terraces in deposition . . . . . . . . . . . . 93

A.1 A kink-flip hop . . . . . . . . . . . . . . . . . 97 
A.2 A pocket-fill hop . . . . . . . . . . . . . . . 97

A.3 A diffusion step . . . . . . . . . . . . . . . . . . 98

A.4 A pinch-plug, starting with 2 neighbours . . . . . . . . . . 98

A.5 A pinch-plug, starting with 3 neighbours . . . . . . . . . . 99

A.6 The ID numbers for the neighbours in the four classes of hop.100 


\section{List of Tables}

3.1 Comparison of IrSC properties with experimental Ir properties. . . . . . . . . . . . . . . . . . 38

3.2 Important Barrier Energies . . . . . . . . . . . . . 46

3.3 Parameterisation of classes of hop ......... 56 


\section{Chapter 1}

\section{Introduction}

\subsection{Epitaxial Growth}

Epitaxial growth is the ordered crystalline growth of a substance deposited on a substrate [1]. It is a method of growing very high quality films, and is useful in any situation where a thin well-ordered film of material is important, such as in the fabrication of microstructures [2] and high temperature superconducting wires [3]. Epitaxy is a slow process - in some circumstances $60 \mathrm{~nm} / \mathrm{min}$ is considered a "high deposition rate" [4] - so it is highly controllable and a complex structure of many very thin layers of different materials can be constructed. This is useful in the semiconductor industry, particularly for optical and microwave frequency electronic devices [5], such as laser diodes [6]. The deposited substance may consist of the same material as the substrate (homoepitaxy) or it may be a different material (heteroepitaxy). Heteroepitaxy can form complex structures, as the stacking type and lattice constants for the substrate may differ from those of the deposited material, while homoepitaxy tends to produce neat layers [1]. The atoms that are absorbed to the surface but are able to move across the surface are called adatoms. The surface and all the layers below the surface is called the substrate. Common methods for epitaxy include chemical vapour deposition (CVD), molecular beam epitaxy (MBE) and 
pulsed laser deposition (PLD).

In CVD, epitaxial layers are formed from chemical reactions with a gas [1]. As the boiling points of most metals and semiconductors are very high, the gas used is some compound which is gaseous at a more convenient temperature. For example, silicon is deposited using gases such as silicon tetrachloride $\left(\mathrm{SiCl}_{4}\right)$, dichlorosilane $\left(\mathrm{SiH}_{2} \mathrm{Cl}_{2}\right)$, trichlorosilane $\left(\mathrm{SiHCl}_{3}\right)$ or silane $\left(\mathrm{SiH}_{4}\right)$. The reactant gases and dopants are transported into a reaction chamber, where they react with the substrate, depositing material. The products are then vented out of the chamber. Typical temperatures for CVD may be in the range $900-1500 \mathrm{~K}$.

In $\mathrm{MBE}$, the epitaxial layers are formed by the reaction of one or more thermal beams of atoms or molecules with the substrate. MBE gives more precise control of chemical compositions and doping profiles than CVD. Growth rates tend to range from 0.001 to $0.03 \mu / \mathrm{min}$ [1]. The substrate temperature is typically about $700-1200 \mathrm{~K}$. The reactants and dopants are housed in effusion ovens within an ultra high vacuum chamber ( $10^{-8} \mathrm{~Pa}$ ). As the material heats, some of it evaporates, and some of the evaporated material will hit the target and be deposited. The very low pressure causes the mean free paths of the evaporated atoms to be larger than geometrical size of the chamber [7]. This means the atoms that hit the target have travelled in a straight line from the source - hence a molecular "beam".

PLD is a relatively new process, only becoming well known in the late 80s [8]. A target is hit by high-powered pulsed laser, vaporising material on the surface to form a plume consisting of a mixture of atoms, molecules, electrons, ions, clusters, micron-sized solid particulates and molten globules. This material moves through the chamber until it encounters the substrate and is deposited. As the plume is highly directional, the chamber may contain a relatively high pressure $(\sim 100 \mathrm{~Pa})$ gas atmosphere such as $\mathrm{O}_{2}$ - for the vaporised material to react with, without reducing the deposition rate significantly, which gives more flexibility in the type of 
materials that can be deposited. For example, a zinc source can be placed in an oxygen atmosphere to cause $\mathrm{ZnO}_{2}$ to be deposited on the substrate. The highly directional plume can cause difficulties with the uniformity of deposition over a large area, which can be reduced through methods such as moving the substrate during the process. The main difficulty with PLD is the phenomenon of "splashing", where the laser causes micron-sized molten globules to emerge from the target which do not stack in a neat epitaxial layer when they strike the substrate. The major advantages of PLD include its simplicity and flexibility.

\subsection{Grain Boundaries}

When epitaxy is performed on the (111) plane of a face-centred-cubic (FCC) substrate, the atoms can be deposited into two major types of site. If only a single layer of close-packed atoms is present, these two sites are equivalent, and differ only by a rotation (Fig. 1.1). If two or more layers are present, these sites are not equivalent. When atoms land in a regular site, they form a pattern that repeats every three (111) layers - referred to as an ABC pattern (see Fig. 1.2). This is a regular FCC structure. There is an alternate class of deposition site, where atoms land in sites directly above the atoms two layers below i.e the structure repeats every second layer an $\mathrm{ABAB}$ pattern (again see Fig. 1.2). These are stacking fault sites, and locally they form a hexagonal-close-packed (HCP) structure. Our simulations focus on an FCC substrate, where deposited atoms may land in HCP or FCC sites. Sometimes the top layer was initially partially HCP, to help us investigate the grain boundaries between HCP and FCC islands.

An adatom placed on the surface of a (111) FCC plane will diffuse over the surface, hopping between stacking fault and regular sites. When adatoms meet, they can aggregate into a small island. If the island is below some critical size it may dissociate, or it may form the fixed nucleus of a stacking fault or regular island [9]. Experiment has shown that for Iridium 


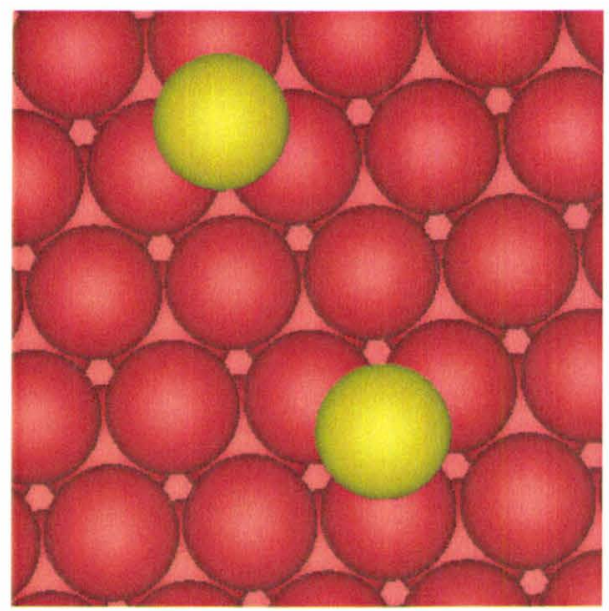

Figure 1.1: Two adatoms on an FCC surface. With respect to the layer beneath the adatoms, both are equivalent, although rotated by $180^{\circ}$. However, the lower atom is directly on top of an atom two layers below (which therefore can not be seen) and so is in an HCP site. The upper atom is not on top of an atom two layers below, and so it is in an FCC site. 

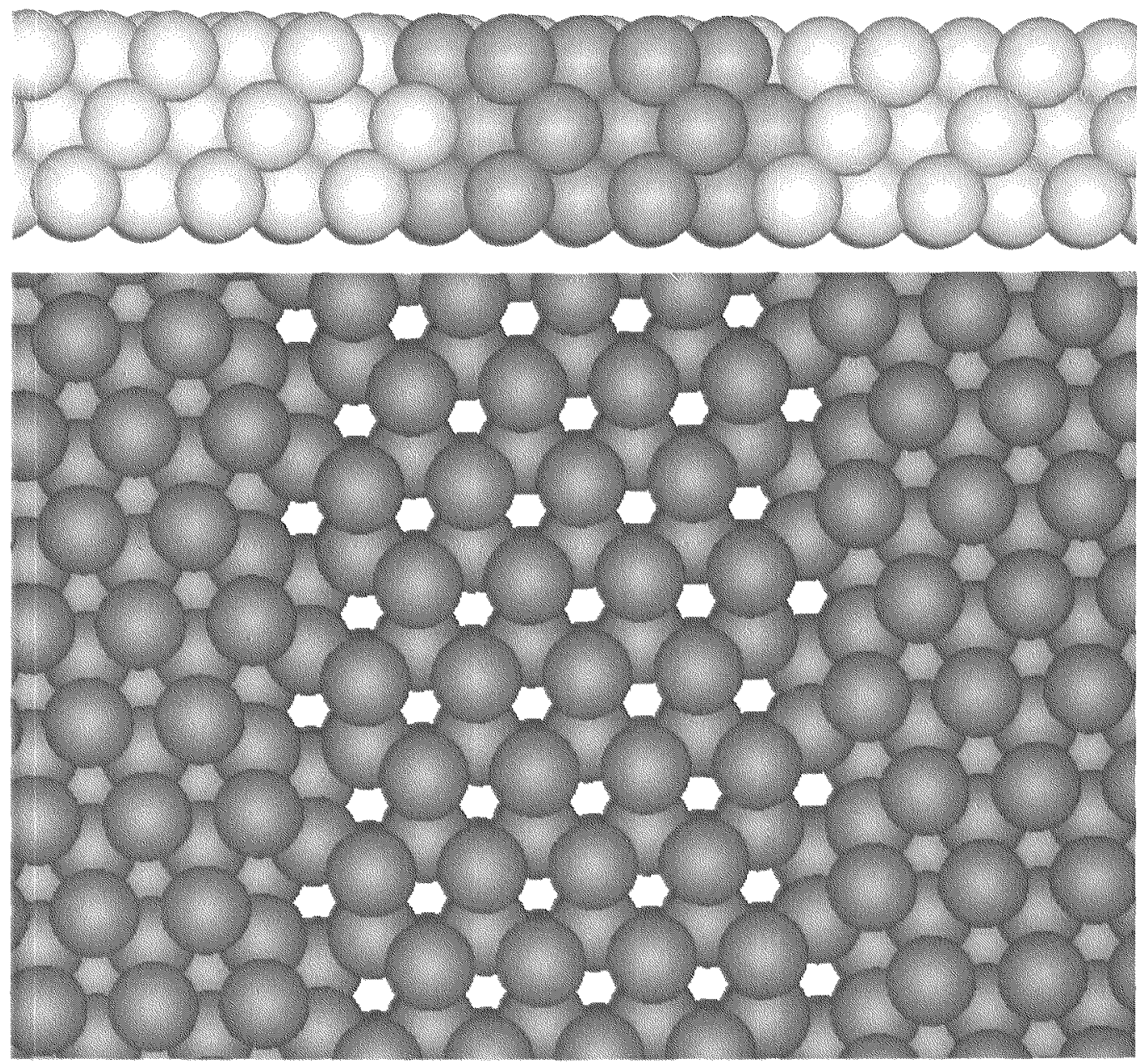

Figure 1.2: Top: 3 layers of (111) planes of an FCC/HCP structure. The dark atoms in the centre are an $\mathrm{ABAB}$ HCP pattern, while the lighter atoms on the left and right have an ABCABC FCC pattern. Below: If we shrink the size of the spheres representing atoms and view the lattice from above, the difference between HCP (centre) and FCC (left and right) structures are clearly visible. The appearance of "gaps" in the HCP structure is because it consists of a pattern that repeats every two layers, instead of every three. 
at $300 \mathrm{~K}$, a group of 4 or more atoms will not diffuse [10]. During epitaxy, a large number of stacking fault and regular islands are nucleated.

Scanning tunnelling microscopy experiments and other KMC simulations [12] show that in Iridium at above $230 \mathrm{~K}$, most of the islands are regular islands, but there are a number of stacking fault islands. Any new atoms that are deposited are more likely to meet and bond to one of these islands than to form a new nucleus, and so the islands grow. Islands "grow true" - that is, an adatom that joins a regular island must take up an FCC site, and adatom that joins a stacking fault island must fill an HCP site. When placing atoms on the surface of a regular FCC slab, it is not possible for an atom in an FCC site to be one nearest neighbour distance from an atom in an HCP site.

These islands generally form dendritic triangular shapes (Fig. 1.3), with stacking fault islands pointing the opposite direction to regular islands. New adatoms may land on top of these islands, and must penetrate a large barrier known as the Ehrlich-Schwoebel barrier $[13,14]$ to hop down the step. Whether adatoms pile on top of islands or have time to hop off to form a flat layer depends on the size of this barrier and the rate of deposition.

As these islands grow, they eventually meet each other. Two islands of the same stacking type that meet will simply amalgamate, but when an HCP (stacking fault) and an FCC (regular) island meet, a grain boundary forms between them, as there is less than a lattice site between the islands - i.e. there is not enough room for an atom to fit. Grain boundaries consist of A-gap and B-gaps (Fig. 1.4). Atoms in an A-gap are so close to each other that there are no valid sites between them and so they are unable to move (with the exception of corner atoms), while atoms on either side of a B-gap have enough room to hop between islands. Where an A-gap meets a B-gap is called a "kink" (Fig. 1.4), and these kinks are important in the movement of grain boundaries. A kink-flip hop (Fig. 1.5, top) gives a mechanism for propagating a grain boundary through A-gaps by mov- 

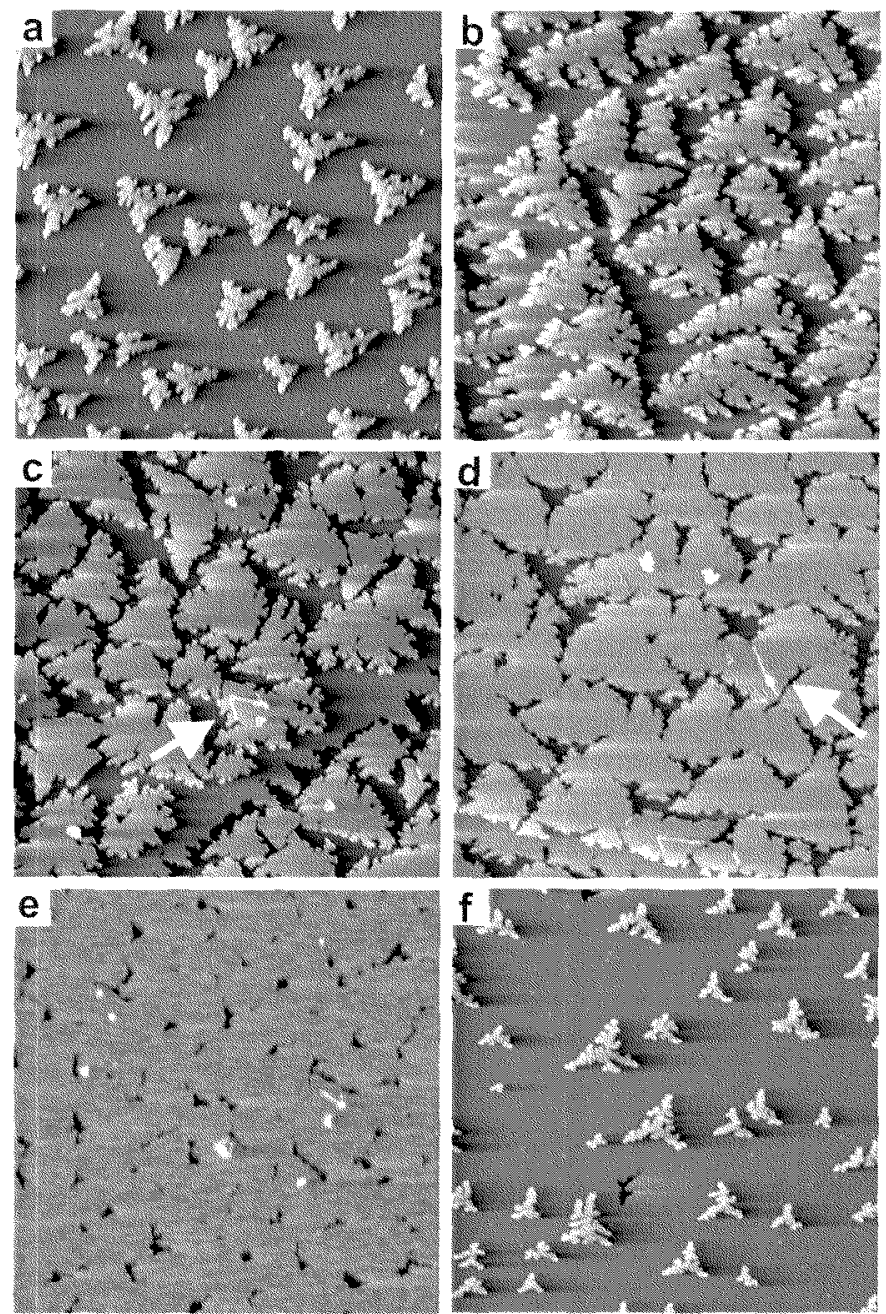

Figure 1.3: A series of STM topographs over a period of $\sim 70 \mathrm{~s}$ of Ir on $\operatorname{Ir}(111)$ at a temperature of $350 \mathrm{~K}$. The picture size is $1100 \AA \times 1100 \AA$. Arrows indicate atoms in decorated (4-fold) sites. Image courtesy of Dr. Carsten Busse at Universität zu Köln. 
ing the A-gap until it encounters another A-gap. Then we are left with an exposed atom which hops quickly through a pocket-fill move (Fig. 1.5, stepping from bottom left to bottom right). Other more complex processes involving concerted movements of several atoms are possible. As a result of these processes, the grain boundary can propagate. It has been observed in scanning tunnelling microscopy (STM) topography of Iridium [15] that the grain boundary tends to propagate in the direction of stacking fault islands, assimilating the HCP islands into FCC and converting the entire surface into an FCC layer, while it has been calculated and shown in experiment [10] that at lower temperatures $(<230 \mathrm{~K}) \mathrm{HCP}$ is preferred instead.

The grain boundary allows another type of site, the decorated site [16]. An atom can sit on an A-gap, supported by four atoms (Fig. 1.3 for STM pictures, Fig. 3.8 (right) for a more schematic view). Atoms in these sites can impede grain boundary movement.

Studying grain boundaries is of interest because it has a significant effect on the quality of thin films. These defects affect the mechanical properties of metals (not only Iridium), making them less malleable by disrupting the motion of dislocations, making deformation more difficult [17]. They also affect the electronic properties - grain boundaries present a barrier that electrons must move around or tunnel through.

\subsection{Atomistic simulations}

It is important to investigate the atomistic processes that occur during epitaxy. However, the wide range of length scales - from small scale interatomic interactions to the formation of many islands on a slab - and the wide range of time scales - from the rapid diffusion of an adatom to the slow growth of a layer - makes analysis difficult. It is difficult for an analytical approach to take into account all the different processes, including deposition, diffusion, island nucleation, island growth and grain boundary propagation. 


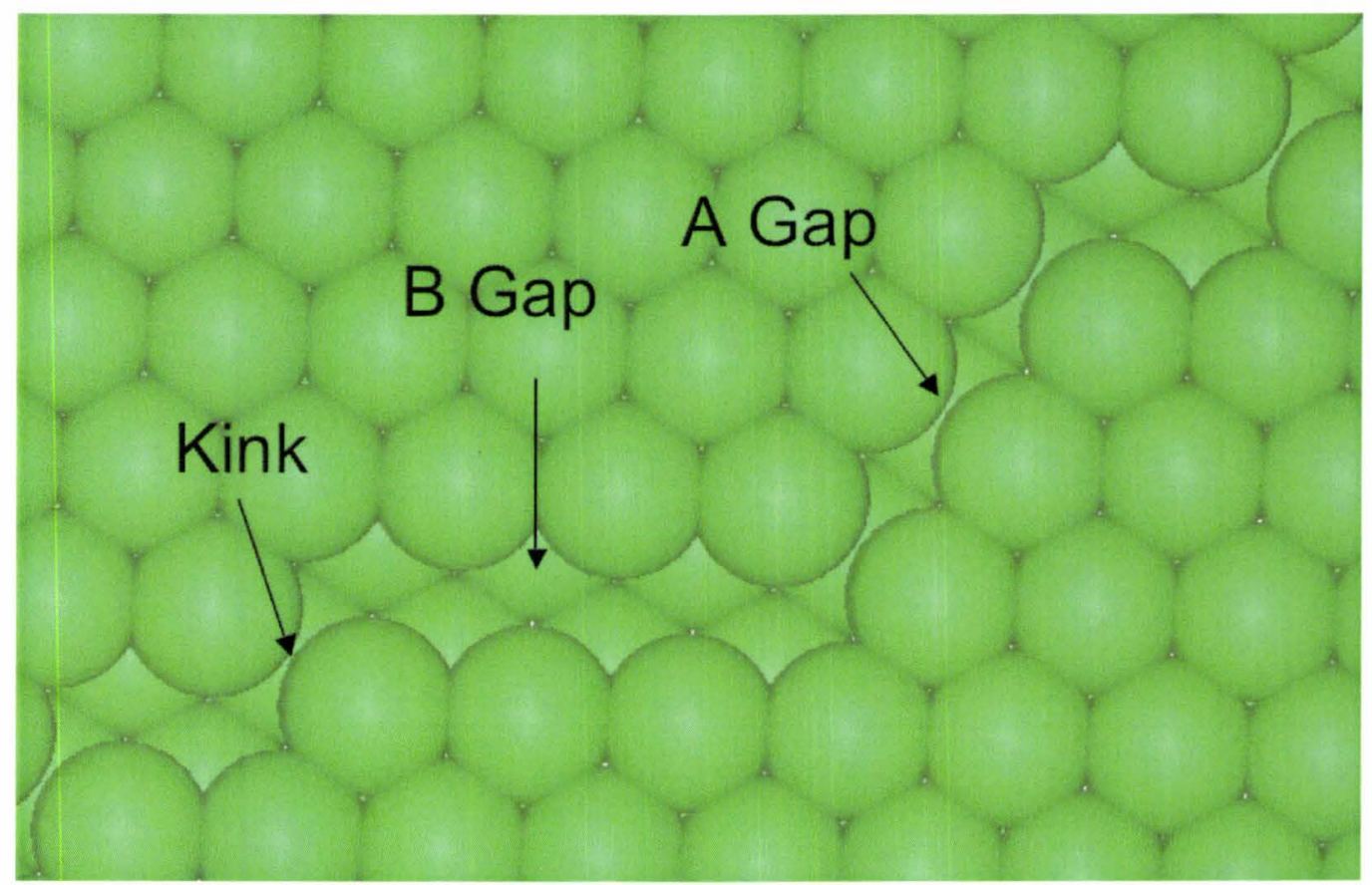

Figure 1.4: A section of a grain boundary containing both A-gaps and Bgaps, and a kink. 

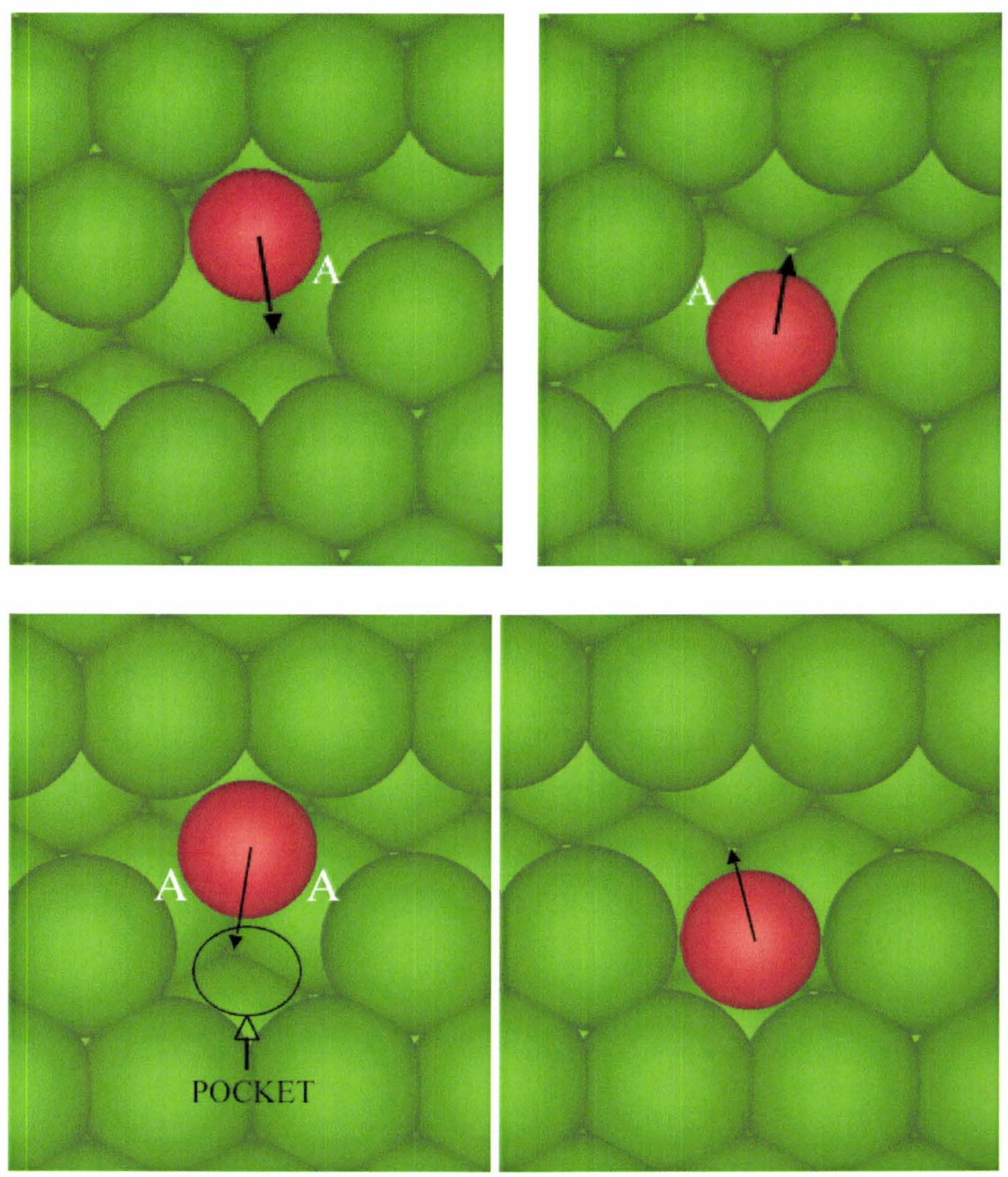

Figure 1.5: The capital " $\mathrm{A}$ " indicates the locations of A-gaps. Above: A kink-flip move. The A-gap moves to the left. The kink-flip can be reversed, moving the A-gap to the right again. Below: A pocket-fill move. Kink-flips have moved A-gaps such that they have merged to form a small pocket. It is a low barrier hop for the atom to hop into this pocket (removing the A-gaps), but a large barrier hop for it to hop out, so it is likely to remain within the pocket. 
Nevertheless, analytical approaches have been attempted - in particular, involving some form of the Burton-Cabrera-Frank (BCF) equations [18]. The original $B C F$ theory describes crystal growth in terms of only a few processes: absorption of adatoms onto terraces and evaporation back into the atmosphere, diffusion of adatoms, and capture of adatoms at step edges. Interactions between adatoms are neglected, although this is an important factor in normal MBE conditions [19]. Later advances have generalised BCF theory to take into account adatom interaction and island nucleation and other complications [20], such as deviations from equilibrium at step edges and the effect of movement of steps. These improvements have renewed interest in the BCF model as a method for describing epitaxy techniques. The disadvantage of an analytical approach such as BCF is that all different processes must be taken into account by the theorist, and each additional process adds complexity to the equations. A simulation technique allows a wider range of behaviour, particularly behaviour that was not expected, and adding additional complexity - such as types of atom hop - is often simple or if the simulation is sufficiently fundamental, not necessary.

Simulation techniques are more capable of taking into account all the details of the problem. Molecular dynamics [21] is one common computational technique for atomistic simulations. Molecular dynamics (MD) is the process of following the trajectories of a collection of atoms by numerical integration of Newton's equations of motion. The forces in these equations are described by some potential function. MD has the advantage that it can simulate a wide variety of processes without them being explicitly prescribed. The main disadvantage is it is inefficient in simulations where the processes have very different rates, or where a large number of atoms are almost stationary. For example, the rate at which an adatom hops into an adjacent site can be many orders of magnitude faster than the rate at which adatoms cross the Ehrlich-Schwoebel barrier. Hence during an epitaxy simulation, an MD algorithm may waste a large amount of time 
integrating the positions of the almost stationary atoms in a substrate in order to diffuse a single adatom over the surface. This will be explained in more detail in Chapter 2.

Kinetic Monte-Carlo (KMC) sacrifices some of the flexibility of MD for a large increase in speed. Many simulations perform KMC on a lattice [22], but off-lattice KMC is also used [23]. In the lattice KMC algorithm, all atoms must be at fixed points on a lattice. The allowed "moves" are hops between these points. Skipping the unstable in-between positions gives a large speed increase over MD, which integrates an atom all the way through a hop. The KMC algorithm determines the rates of all possible hops for each atom, and each iteration randomly selects one of these hops (weighted according to its rate) and performs it. The time step is determined according to the number of possible hops, and so will appropriately increase if there are not many processes occurring and decrease if there are many fast processes. This makes KMC much more efficient than MD.

The main disadvantage of lattice KMC is that all possible types of hop must be predetermined. An MD simulation may perform some unexpected rare move, but unless the particular implementation of $\mathrm{KMC}$ has this move coded into it, KMC can not perform it. For example, true dimer diffusion is not possible if the KMC code can only perform single atom hops. Nevertheless, lattice KMC can perform complex behaviour based on a small number of relevant moves.

Off-lattice KMC permits atoms to take any position over a continuous space. The possible hops (to nearby energy minima) and their energy barriers (at energy saddle points) are calculated using a method such as the dimer method [24] during the KMC simulation. Off-lattice KMC is more flexible than on-lattice $\mathrm{KMC}$, as it permits a wider variety of hops as they do not need to be calculated beforehand. However, calculating the hops during the process slows down the algorithm. Hence off-lattice KMC lies between MD and on-lattice KMC in terms of speed and flexibility. 
Hybrid processes involving a combination of KMC or MD have been developed [25]. In this approach, the regions around the grain boundaries are simulated by MD to capture the details of the process occurring there, while the rest of the slab is simulated with KMC. The boundaries of these regions dynamically change as the system evolves, to follow the grain boundaries. This gives a compromise between the two methods used - trading some speed for a large gain in accuracy. We use a straight KMC method as our main algorithm, as its speed allows us to achieve a large number of varied simulations. We use the Nudged Elastic Band technique (NEB) combined with MD to calculate the barrier energies we need as input for $\mathrm{KMC}$, but no MD simulations are performed during the $\mathrm{KMC}$ simulation.

\subsection{Outline of the following chapters}

\subsubsection{Chapter 2: Methodology}

Chapter 2 describes the general techniques used - namely, Molecular Dynamics (Section 2.1), Nudged Elastic Band (Section 2.2) (including relaxation algorithms (Section 2.2.1) such as FIRE, the Fast Inertial Relaxation Engine [26]) and Kinetic Monte-Carlo (Section 2.3). It includes discussions on the merits and disadvantages of these methods.

\subsubsection{Chapter 3: Implementation}

Chapter 3 contains a more detailed explanation of the particular methods used.

Section 3.1 explains the Molecular Dynamics implementation, such as the Velocity Verlet integration technique (subsection 3.1.1), our choice of intermolecular potentials, Lennard-Jones and Iridium Sutton-Chen (subsection 3.1.2) and our experience with the FIRE [26] relaxation algorithm 
(subsection 3.1.3). It also contains details of our implementation of the Nudged Elastic Band technique (subsection 3.1.4), including the configuration of the systems we examined, and the results and analysis of the energy barriers calculated with the Nudged Elastic Band technique (subsection 3.1.5). The NEB technique is found to be consistent to a level of about $0.01 \epsilon$ or $0.01 \mathrm{eV}$, larger than the energy difference between FCC and HCP hops that we calculated.

Section 3.2 explains details of the Kinetic Monte-Carlo implementation. Subsection 3.2.1 explains our particular representation of the lattice in the Kinetic Monte-Carlo algorithm, section 3.2.2 explains our "look-up table" method of identifying hops according to the location of their nearest neighbours, subsection 3.2.3 describes the particular data structures and algorithms used to make our code efficient, and subsection 3.2.4 lists other details, including how deposition is handled. Section 3.3 describes the test cases of adatom diffusion used to determine the KMC pre-factor.

\subsubsection{Chapter 4: Results and Analysis}

Chapter 4 gives the results of our Kinetic Monte-Carlo simulations.

Section 4.1 describes our simulations on the propagation of grain boundaries in systems with a zero or near-zero deposition rate. The merger of grain boundaries and the sensitivity of the direction of grain boundary propagation to small differences in energy between HCP to FCC and FCC to HCP hops is described. Also, the effect of adatoms on the surface (to simulate a non-zero deposition rate) is examined.

Section 4.2 describes the results of deposition simulations, particularly concerning the structure of islands formed.

\subsubsection{Chapter 5: Conclusions}

Chapter 5 summarises and discusses the results and methods of the previous sections. 


\subsubsection{Appendix A}

This appendix gives diagrams of all the important hops referred to in the main body of the dissertation, for reference. 


\section{Chapter 2}

\section{Methodologies}

This chapter describes the methodologies we used and justification for our decisions to use these methodologies.

There is a large variety of techniques that can be used to model atomic systems. Our method is based on the on-lattice Kinetic Monte-Carlo (KMC) algorithm. This algorithm requires fore-knowledge of the rates of all possible processes (hops or depositions). These can be calculated through transition state theory, provided the activation energies for the processes are known. We calculated these using the Nudged Elastic Band (NEB) method, a technique for finding the minimum energy path (and hence the barrier energy) for a process. NEB requires a series of duplicates (or "images") $^{\prime \prime}$ of the system interpolated between the start and endpoints of the hop to be relaxed under the constraint of an "elastic band" force. We simulated these images using Molecular Dynamics, and relaxed them with the recently-developed FIRE (Fast Inertial Relaxation Engine) [26] algorithm.

In section 2.1 we describe the principles behind Molecular Dynamics (MD) and what we used it for. MD is a method of evolving a system of particles by integrating Newton's equations of motion. The forces on the particles are calculated using empirical potentials. MD permits a large variety of behaviour, but is too slow for simulating very large numbers of particles (See section 1.3). We used MD as part of the NEB method to 
calculate the barrier energies of hops, but not to simulate the entire system.

In section 2.2 we describe the NEB technique and corresponding relaxation techniques. These are methodologies for finding the minimum energy path for a transition, and hence the barrier energy for that transition. It involves setting up a system of "images" interpolated between the initial and final configuration, and relaxing these images while subject to a spring force to keep them in a line. We also describe the FIRE algorithm for relaxing the images.

In section 2.3 we describe the general KMC algorithm. KMC is based on knowing the rates for all events in a system. One of these events is randomly selected (weighted according to its rate) and performed each time-step. This is a very efficient method of evolving a system, but is not as generic as MD. However, it is fast enough to simulate large numbers of atoms, and this is the method we use to simulate grain boundary propagation and epitaxial growth.

\subsection{Molecular Dynamics}

The background information in this section is largely based on [27]. The principles behind Molecular Dynamics (MD) are simple. We set up a system of particles which are treated classically, with each having a definite mass, position, velocity and acceleration. The acceleration is given by a potential function. The potential function determines how particles interact, and so its exact form will depend on what elements are being simulated. A simple potential function is the Lennard-Jones (LJ) potential function, where the energy of the system is equal to

$$
E_{\text {total }}=4 \epsilon \sum_{i} \sum_{j \neq i}\left[\left(\frac{\sigma}{r_{i j}}\right)^{12}-\left(\frac{\sigma}{r_{i j}}\right)^{6}\right]
$$

where $r_{i j}$ is the distance between particles $i$ and $j$ and $\sigma$ and $\epsilon$ are length and energy scales respectively. The LJ potential is an approximation, and 
is most accurate for noble gases, but is not generally considered appropriate for modelling metals. It contains a long-range attractive part (the van der Waals force) and a short range repulsive potential (Pauli repulsion). The LJ potential is called a two-body potential as is it the sum of interactions between all combinations of two particles. More sophisticated potentials take into account many-body interactions due to electrons forming bonds and other important behaviour, and predict more realistic behaviour for metals and other materials.

The force on a particle is evaluated by taking the gradient of the potential function with respect to that particle's position. In practice, the gradient as a function of particle position is usually worked out by hand or with symbolic manipulation software such as Maple, and numerical values for particle position are inserted when the simulation is run. However sometimes - such as when the potential is a spline fit to empirical data the derivative may be entirely numerically evaluated.

With this force we can calculate the acceleration. Then, given an initial configuration of positions and velocities of the particles, we can use numerical integration techniques to estimate their positions and velocities after a small time step $d t$ has elapsed. This process is repeated to evaluate the evolution of the system over some period of time.

Numerical integration techniques are always approximations, and so numerical errors will always be present in an MD simulation. By keeping the time step small enough, we can reduce the magnitude of these errors. However, the smaller the time step is, the more calculations are necessary to evolve the system over a certain period of simulated time, and thus the more computer time is required. So an optimal time step is a compromise between efficiency and accuracy.

Typically, the strength of interaction described by a inter-atomic potentials drops off rapidly with distance, such as the $\frac{1}{r^{6}}$ tail of the LJ potential. Hence the interactions between particles beyond some distance is negligible and can be ignored to speed up computation time. Beyond a certain 
distance known as the "cut-off distance", all interactions are ignored. This cut-off distance needs to be large enough that it does not change the behaviour of the system, but small enough to cut out enough interactions to speed up the calculation sufficiently. Typical values for $L J$ are $3 \sigma$. This cutoff can cause a small discontinuity in the potential function which may cause energy to not be conserved in the simulation. To prevent this, the potential can be modified to ensure it and at least its first derivative are continuous over the cut-off.

Molecular dynamics simulations can typically simulate systems for a time-scale of nanoseconds with current computing power. Simulating slow processes such as deposition are computationally expensive. For example the computer may spend many iterations calculating the effect that a single adatom diffusing over a surface has on all the nearby atoms in the substrate. The processor time for a simple MD algorithm with a two-body interaction scales as $O\left(N^{2}\right)$, where $N$ is the number of particles in the system. Sophisticated improvements, including Verlet lists, and cell methods [21] can improve this up to $O(N)$. We use MD as part of the NEB method to simulate individual hops to find out the barrier energies that we use for kinetic Monte-Carlo (Section), but not to simulate the entire system.

\subsection{Nudged Elastic Band}

Much of this section is based on the review article [28]. The Nudged Elastic Band technique is a method for finding the minimum energy path (MEP) between two states of a system. This is the path the system will take between these two states. The difference between the point with the highest energy along this path (the highest saddle point) and the energy of the initial configuration is the barrier energy or activation energy, the energy required for the system to make the transition from the first state to the second. In our case, this transition is the hop of a single atom.

The NEB method works by producing several copies or "images" of 
the system, interpolated between the final and initial states. Each of these images is then relaxed, possibly using an MD-based technique such as Simulated Annealing [29] or FIRE [26], while an "elastic band" force is applied to prevent all the images falling into the initial or final states and to compel each image to stay between its two adjacent images and form a smooth path. The simplest form of this force is

$$
\vec{F}_{i}=-\vec{\nabla} V\left(\vec{R}_{i}\right)+\vec{F}_{i}^{s}
$$

where

$$
\vec{F}_{i}^{s}=k_{i+1}\left(\vec{R}_{i+1}-\vec{R}_{i}\right)-k_{i}\left(\vec{R}_{i}-\vec{R}_{i-1}\right)
$$

where $\vec{F}_{i}$ the global force vector for image $i, V\left(\vec{R}_{i}\right)$ is the potential energy vector for image $i$ for position global position vector $\vec{R}_{i}$, calculated in the normal way (e.g. by interactions between particles in the same image), $\vec{F}_{i}^{s}$ is the global spring force vector on image $i$, which is determined by the positions vectors of images $i+1, i$ and $i-1-\vec{R}_{i+1}, \vec{R}_{i}$ and $\vec{R}_{i-1}$ respectively, and the spring constants for image $i, k_{i}$ and image $i+1, k_{i+1}$.

Relaxing the images with this simple elastic band force gives an approximation to the MEP. In our case, the MEP corresponds to the paths of the atoms when one atom makes a hop. The difference between the energy of the image with the greatest energy and the energy of the end points gives an estimate for the barrier energy for this hop in each direction. However, this simple method does not converge well on the MEP it tends to cut corners. Also, it is quite possible that the maximum energy will occur between images, so a large number of images are required to measure the barrier energy with any precision. There are various modifications that fix these problems. The first improvement is replacing the elastic band force with the Nudged Elastic Band (NEB) force,

$$
\vec{F}_{i}=-\left.\vec{\nabla} V\left(\vec{R}_{i}\right)\right|_{\perp}+\left.\vec{F}_{i}^{s}\right|_{\|}
$$




$$
=-\left.\vec{\nabla} V\left(\vec{R}_{i}\right)\right|_{\perp}+\left(\vec{F}_{i}^{s} \bullet \hat{\tau}\right) \hat{\tau}
$$

where $\hat{\tau}$ is the unit tangent to the path and $\left.\vec{\nabla} V\left(\vec{R}_{i}\right)\right|_{\perp}=\vec{\nabla} V\left(\vec{R}_{i}\right)-\left(\vec{\nabla} V\left(\vec{R}_{i}\right) \bullet\right.$ $\hat{\tau}) \hat{\tau}$. $\hat{\tau}$ can be determined by the normalised line segment between the previous and following image,

$$
\hat{\tau}_{i}=\frac{\vec{R}_{i+1}-\vec{R}_{i-1}}{\left|\vec{R}_{i+1}-\vec{R}_{i-1}\right|}
$$

but it is slightly better to bisect the two unit vectors [30]

$$
\vec{\tau}_{i}=\frac{\vec{R}_{i}-\vec{R}_{i-1}}{\left|\vec{R}_{i}-\vec{R}_{i-1}\right|}+\frac{\vec{R}_{i+1}-\vec{R}_{i}}{\left|\vec{R}_{i+1}-\vec{R}_{i}\right|}
$$

and then to normalise $\hat{\tau}=\frac{\vec{\tau}}{|\vec{\tau}|}$. This splitting into parallel and perpendicular components is what is referred to by "nudging". This prevents the true force and the spring force from competing. At all points along the minimum energy path $\left.\vec{\nabla} V\left(\vec{R}_{i}\right)\right|_{\perp}=0$, and the NEB images converge towards this.

Unfortunately, in conditions where the force parallel to the MEP is large compared to the force perpendicular to the MEP and many images are used, oscillating kinks can develop in the elastic band. To illustrate the origin of kinks, we examine the simple situation illustrated in Fig. 2.1. We assume there is a constant force $F$ in the "down" direction, as well as a restoring force of $-d x C$ perpendicular to the MEP, which we assume to be straight, where $d x$ is the perpendicular distance from the MEP and $C$ is the curvature of the potential energy surface.

The atom in the middle image in Fig. 2.1 has been displaced a small distance $d x$ and hence experiences a restoring force $d x C$. However, using the tangent estimate above (Eq. 2.7), the tangent for the neighbouring image with higher energy is no longer along the MEP, and the force $F$ gives it a small perpendicular force $d x F / 2 R$, where $R$ is the spacing between the images. The band is only stable if the destabilising force $d x F / 2 R$ is smaller than the restoring force $d x C$, that is 


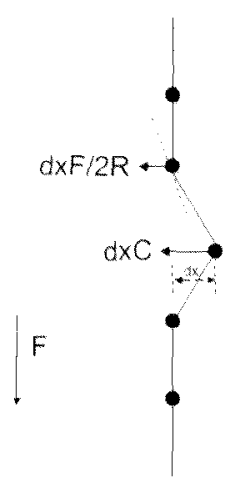

Figure 2.1: A segment of an elastic band, demonstrating the origin of kinks. The dots indicate the locations in each image of a particular atom of interest. There is a constant "downwards" force $F$. An image is displaced a small distance $d x$ and experiences a force $d x C$. This causes the higherup image to experience a force $d x F 2 R$, where $R$ is the spacing between images. 


$$
F<2 C R \text {. }
$$

As this is dependent on the number of images in the band, the band will always become unstable if it consists of enough images.

One suggestion to alleviate this instability is to use a smooth switching function that gradually turns on the perpendicular component of the spring force when the path becomes kinky. The force on an image is then given by

$$
\vec{F}_{i}^{N E B}=\vec{F}_{i}^{0}+f\left(\phi_{i}\right)\left(\vec{F}_{i}^{S}-\vec{F}_{i}^{S} \bullet \hat{\tau} \hat{\tau}\right)
$$

where $f(\phi)$ is some function which goes from 0 for a straight path up to 1 for a right angle, e.g.

$$
f(\phi)=\frac{1}{2}(1+\cos (\pi \cos (\phi)))
$$

for $|\phi|<\pi / 2$ and 1 elsewhere.

Another suggestion is to use an improved tangent estimate [30]. Here

$$
\vec{\tau}_{i}= \begin{cases}\vec{\tau}_{i}^{+}, & \text {if } V_{i+1}>V_{i}>V_{i-1} . \\ \vec{\tau}_{i}^{-}, & \text {if } V_{i+1}<V_{i}<V_{i-1}\end{cases}
$$

where $\vec{\tau}_{i}^{+}=\vec{R}_{i+1}-\vec{R}_{i}$ and $\vec{\tau}_{i}^{-}=\vec{R}_{i}-\vec{R}_{i-1}$. However, this leads to an abrupt change in tangents at extrema along the MEP which may cause convergence problems. Hence if image $i$ is at a maximum $V_{i+1}<V_{i}>V_{i-1}$ or a minimum $V_{i+1}>V_{i}<V_{i-1}$, the tangent estimate becomes

$$
\vec{\tau}_{i}= \begin{cases}\vec{\tau}_{i}^{+} \Delta V_{i}^{\max }+\tau_{i}^{-} \Delta V_{i}^{\min }, & \text { if } V_{i+1}>V_{i-1}, \\ \vec{\tau}_{i}^{+} \Delta V_{i}^{\min }+\tau_{i}^{-} \Delta V_{i}^{\max ,}, & \text { if } V_{i+1}<V_{i-1},\end{cases}
$$

where

$$
\Delta V_{i}^{\max }=\max \left(\left|V_{i+1}-V_{i}\right|,\left|V_{i-1}-V_{i}\right|\right)
$$

and 


$$
\Delta V_{i}^{\min }=\min \left(\left|V_{i+1}-V_{i}\right|,\left|V_{i-1}-V_{i}\right|\right) .
$$

The tangent is weighted according to the energies of the adjacent images, providing a smooth transition. Henkelman [30] also suggests changing the spring force to

$$
\left.\vec{F}_{i}^{s}\right|_{\|}=k\left(\left|\vec{R}_{i+1}-\vec{R}_{i}\right|-\left|\vec{R}_{i}-\vec{R}_{i-1}\right|\right) \hat{T}_{i}
$$

instead of equation 2.3. Provided $k$ is constant, this ensures equal spacing of the images even when the angle between $\vec{R}_{i+1}-\vec{R}_{i}$ and $\vec{R}_{i}-\vec{R}_{i-1}$ is large.

The climbing image nudged elastic band method [31] is an improvement to ensure one of the images is directly on top of the maximum energy along the MEP, and so the value we record is indeed the barrier energy. After a certain number of iterations, or after the barrier energy has sufficiently converged, one (or more) image is designated the climbing image. The force on this one image is determined by

$$
\vec{F}_{i_{\max }}=-\nabla E\left(\vec{R}_{i_{\max }}\right)+2 \nabla E\left(\vec{R}_{i_{\max }}\right) \bullet \hat{\tau}_{i_{\max }} \hat{\tau}_{i_{\max }}
$$

instead of the normal combination of spring force and true force. This is the full regular force with the part parallel to the elastic band reversed. The unchanged perpendicular part of the force ensures the image still converges on the MEP, while the reversed parallel part lets the image climb up to the highest energy along the MEP. As the highest energy along the path is what we are generally most interested in, typically it is preferable to have a higher resolution in the MEP around this point. This can be achieved with variable spring "constants". For each image $i$ we set the spring constant to

$$
k_{i}^{\prime}=\left\{\begin{array}{cl}
k_{\max }-\Delta k\left(\frac{E_{\max }-E_{i}}{\left.E_{\max }-E_{r e f}\right),}\right. & \text { if } E_{i}>E_{r e f}, \\
k_{\max }-\Delta k, & \text { if } E_{i}<E_{r \in f},
\end{array}\right.
$$


where $E_{i}=\max \left(E_{i}, E_{i-1}\right) . E_{\text {ref }}$ is chosen to be the energy of the higher energy endpoint of the MEP.

\subsubsection{Relaxation Methods}

Simulated Annealing and the Fast Inertial Relaxation Engine (FIRE) are both MD based methods for relaxing a system to its minimum energy state. Simulated Annealing starts the system at some fixed temperature, and then reduces this temperature, scaling the velocities of the particles in the system to match. The process is "annealing" rather than "quenching" as the system is cooled slowly enough for it to explore the energy landscape and find the absolute minimum instead of going straight down to the closest minimum. As the temperature approaches zero, the system approaches its most relaxed state [29].

FIRE acts by applying an artificial "friction force" in the direction of motion of the atoms, and applying an acceleration in the "downhill" direction of the energy landscape. It also freezes the system (i.e. $v=0$ for all atoms) when the system goes "uphill" (i.e. the total energy increases), and takes advantage of variable time-steps [26].

This is implemented mathematically by the statement that a "blind skier" searching for the fastest way to the minimum in an unknown potential landscape should follow the following equation of motion:

$$
\dot{\mathbf{v}}=\mathbf{F} / m-\gamma(t)|\mathbf{v}(t)|[\hat{\mathbf{v}}(t)-\hat{\mathbf{F}}(t)] .
$$

Given initial values for $\Delta t, \alpha=\alpha_{\text {start }}$, for global vectors $\mathbf{x}$ and $\mathbf{v}=0$ and for parameters $N_{\text {min }}, f_{\text {ine }}, f_{\text {dec }}, \alpha_{\text {start }}$ and $f_{\alpha}$, this is implemented discretely with the following algorithm:

MD: calculate $\mathbf{x}, \mathbf{F}=-\nabla E(\mathbf{x})$, and $\mathbf{v}$ using any common MD integrator; check for convergence.

F1: calculate $P=\mathbf{F} \cdot \mathbf{v}$. 
F2: set $\mathbf{v} \rightarrow(1-\alpha) \mathbf{v}+\alpha \hat{\mathbf{F}}|\mathbf{v}|$.

F3: if $P>0$ and the number of steps since $P$ was negative is larger than $N_{\text {min }}$, increase the time step $\Delta t \rightarrow \min \left(\Delta t f_{\text {inc }}, \Delta t_{\text {max }}\right)$ and decrease $\alpha \rightarrow \alpha f_{\alpha}$. That is, if the system is evolving in the right direction (towards lower energy), and has been doing so for the last few iterations, increase the time step and lower the friction force to reduce the number of iterations before a turning point is encountered.

F4: if $P \leq 0$, decrease the time step $\Delta t \rightarrow \Delta t f_{\text {dec }}$, freeze the system $\mathbf{v} \rightarrow 0$ and set $\alpha$ back to $\alpha_{\text {start }}$. In other words, if the system is evolving in the wrong direction - trying to go "uphill" - stop all atoms, and lower the time step and increase the friction force to ensure any nearby turning points are not skipped over.

F5: return to $\mathrm{MD}$.

As FIRE is essentially a quenching method, taking the fastest downhill path to to a local minimum, we would expect it to be more likely to find a local minimum than Simulated Annealing, which explores the parameter space more fully is more likely to reach the global minimum [36]. Hence Simulated Annealing should give a better estimate of the MEP. After performing NEB runs under both Simulated Annealing and FIRE, we noticed the difference in energy barriers between the two methods was too small to be significant for our purposes - less than $0.01 \epsilon$ or $0.01 \mathrm{eV}$, the variation of our NEB code on initial conditions. FIRE was much faster than Simulated Annealing so we selected FIRE as the relaxation algorithm for our NEB code. 


\subsection{Kinetic Monte-Carlo and transition state the- ory}

Kinetic Monte-Carlo is a fast stochastic simulation technique. It is commonly used in simulations of deposition and crystal growth [34, 35], as well as various forms of diffusion $[37,38,39]$.

According to transition state theory [32], the rate law for a process with barrier (or "activation") energy $E_{b}$,

$$
r=A N \exp \left(-\frac{E_{b}}{k_{B} T}\right)
$$

where $A$ is a pre-factor, $N$ is the number of possible ways this process could occur (i.e. from different orientations and different atoms), $k_{B}$ is Boltzmann's constant and $T$ is the temperature, which we assume to be constant throughout the system. The pre-factor $A$ is largely an "attempt frequency" for the hop, and so should be of the order of the atomic vibration frequency $\left(\sim 10^{-13} \mathrm{~Hz}[18]\right)$, but also includes entropy considerations. The energy barrier $E_{b}$ can be calculated using a method such as the dimer [24] method or NEB, or by assuming it is proportional to the number of initial neighbours [33].

This is assuming classical Boltzmann statistics [40], a low temperature approximation, assuming that $k_{B} T \ll E_{b}$. If $k_{B} T \sim E_{b}$ then the simple $\exp \left(E_{b} / k_{B} T\right)$ factor is no longer valid, and we have to take into account Fermi-Dirac or Bose-Einstein statistics to achieve realistic results. For systems at $1000 \mathrm{~K}$, the greatest temperature we used, this means we need $E_{b}$ to be greater than $0.08 \mathrm{eV}$.

In our case, the processes are either single atom hops between nearby sites or deposition events.

The Kinetic Monte-Carlo (KMC) algorithm is based on knowing the rates of all relevant hops, whether calculated from transition state theory or otherwise, and is as follows: 
1. Calculate all the possible transitions and their rates.

2. Randomly select a transition, where the probability of each transition is proportional to its rate.

3. Perform this transition and increment the time by the time-step

4. Repeat $1-3$

The time-step must be determined every iteration. It can be simply

$$
d t=\frac{1}{\sum_{i} r_{i}}
$$

where $r_{i}$ is the rate of process $i$, and the sum is over every process every atom can perform. This intuitively makes sense - if we have a process with a rate of $1 \mathrm{~Hz}$, and another process with a rate of $4 \mathrm{~Hz}$, we would expect on average over a long period of time the rate of any event occurring to be 5 $\mathrm{Hz}$, i.e. every $\frac{1}{1+4}=0.2 \mathrm{~s}$. An approach more faithful to KMC's stochastic nature is to generate a uniform random number $x \in(0,1]$ and use the formula

$$
d t=-\frac{\ln x}{\sum_{i} r_{i}}
$$

which has the same average value over a large enough number of iterations. For large values of $N, \sum_{i}^{N} \ln x$ tends to $N$ and we generally save a copy of the system on the order of at least $10^{3}$ or $10^{4}$ iterations (or more depending on the situation being simulated). The save frequency was chosen to save about 20-100 copies of the system over the simulation run, spaced evenly by simulation time (not by number of iterations). As the number of iterations between saves is not large, we do not expect a major difference between these approaches. Hence we use the simpler non-stochastic method. 


\section{Chapter 3}

\section{Implementation}

The methods detailed in the previous chapter are quite general methods that can model a variety of situations in a variety of ways. In this chapter we explains our particular implementations that we found useful for modelling grain boundary propagation and epitaxy. Although most of our choices of implementation are based on previous work, doubling the KMC mesh resolution to allow decorated sites, and our particular KMC look-up table are novel to our approach. The FIRE relaxation algorithm [26] and the Schulze KMC algorithm [41] have only been reported recently and have not been widely implemented yet.

Section 3.1 explains our MD based methods for finding the barrier energies, as required by KMC. Subsection 3.1.1 derives our choice of integration algorithm, Velocity Verlet. Subsection 3.1.2 describes the potentials we used - both the traditional Lennard-Jones potential, and the SuttonChen potential, a simple Embedded Atom Method potential with parameters for Iridium. Subsection 3.1.3 gives the parameters we used for the FIRE algorithm, and our experiences with this recently developed method. A more detailed description of the FIRE algorithm is in section 2.2.1 in the previous chapter. Subsection 3.1.4 gives details about the overall NEB method, including the configuration of atoms used in the hops, convergence checks, and dealing with hops to or from unstable sites. Subsection 
3.1.5 gives a description of the barrier energies determined by NEB, and we show that a simple neighbour-counting method for determining barrier energies in $\mathrm{KMC}$ is not sufficient, and that a method such as a look-up table is necessary.

Section 3.2 details our particular implementation of KMC and the lattice it is performed on. Subsection 3.2.1 gives an argument for doubling the triangular mesh size to allow atoms to sit in a decorated row, and gives details on the lattice geometry and the four classes of possible hop - small hops, drop hops, entering/exiting a decorated site, and moving from one decorated site to another. Subsection 3.2.2 explains our lookup table method for matching potential KMC moves with energy barriers calculated with NEB. Subsection 3.2.3 gives our implementation of the Schulze algorithm for KMC [41]. Subsection 3.2.4 gives other details in the KMC implementation, including the pre-factor and how deposition is treated and evaporation is ignored. In subsection 3.3 we determine the $\mathrm{KMC}$ pre-factors by comparing KMC adatom diffusion with experimental and calculated data.

\subsection{Molecular Dynamics}

\subsubsection{Integration with Velocity Verlet}

We used Velocity Verlet [27] as our integration technique. The simple Verlet scheme is derived by taking 3rd order Taylor expansions about position at time $t$,

$$
\begin{aligned}
& \vec{r}(t+\Delta t)=\vec{r}(t)+\vec{v}(t) \Delta t+(1 / 2) \vec{a}(t) \Delta t^{2}+(1 / 6) \vec{j}(t) \Delta t^{3}+O\left(\Delta t^{4}\right) \\
& \vec{r}(t-\Delta t)=\vec{r}(t)-\vec{v}(t) \Delta t+(1 / 2) \vec{a}(t) \Delta t^{2}-(1 / 6) \vec{j}(t) \Delta t^{3}+O\left(\Delta t^{4}\right)
\end{aligned}
$$

where $\vec{r}$ is the position, $\vec{v}$ is the velocity, $\vec{a}$ is the acceleration and $\vec{j}$ is the jerk of the particle. Adding the two expressions gives 


$$
\vec{r}(t+\Delta t)=2 \vec{r}(t)-\vec{r}(t-\Delta t)+\vec{a}(t) \Delta t^{2}+O\left(\Delta t^{4}\right)
$$

which is the basic equation behind the Verlet algorithm. However, this method does not explicitly give velocity at time $t+\Delta t$, which is necessary for FIRE, so we use the Velocity Verlet scheme instead, where

$$
\begin{array}{r}
\vec{r}(t+\Delta t)=\vec{r}(t)+\vec{v}(t) \Delta t+(1 / 2) \vec{a}(t) \Delta t^{2} \\
\vec{v}(t+\Delta t / 2)=\vec{v}(t)+(1 / 2) \vec{a}(t) \Delta t \\
\vec{a}(t+\Delta t)=-(1 / m) \nabla V(\vec{r}(t+\Delta t)) \\
\vec{v}(t+\Delta t)=\vec{v}(t+\Delta t / 2)+(1 / 2) \vec{a}(t+\Delta t) \Delta t
\end{array}
$$

which gives equivalent trajectories to the Verlet algorithm to $O\left(\Delta t^{2}\right)$.

\subsubsection{Potentials}

We used two potentials to describe the interactions between atoms, the Lennard-Jones (LJ) potential [42] and an Iridium Sutton-Chen (IrSC) potential [43]. A comparison of the two potentials is plotted in Fig. 3.1. The IrSC potential has a longer ranged effect than the LJ potential.

The LJ potential is of the form

$$
E_{\text {total }}=4 \epsilon \sum_{i} \sum_{j \neq i}\left[\left(\frac{\sigma}{r_{i j}}\right)^{12}-\left(\frac{\sigma}{r_{i j}}\right)^{6}\right] .
$$

where $\sigma$ is a distance scale and $\epsilon$ is an energy scale. It is common practice [27] to use reduced units where $\sigma=1, \epsilon=1$ for computational calculations and only to convert to "real" units at the end, and then only if necessary. We used a cut-off distance of $6 \sigma$, instead of the more common $\sim 3 \sigma$ as we wanted to ensure each atom "felt" the difference between FCC and HCP structure.

The Sutton-Chen potential is of the Embedded-atom (EAM) [44] class of potentials, of the form 


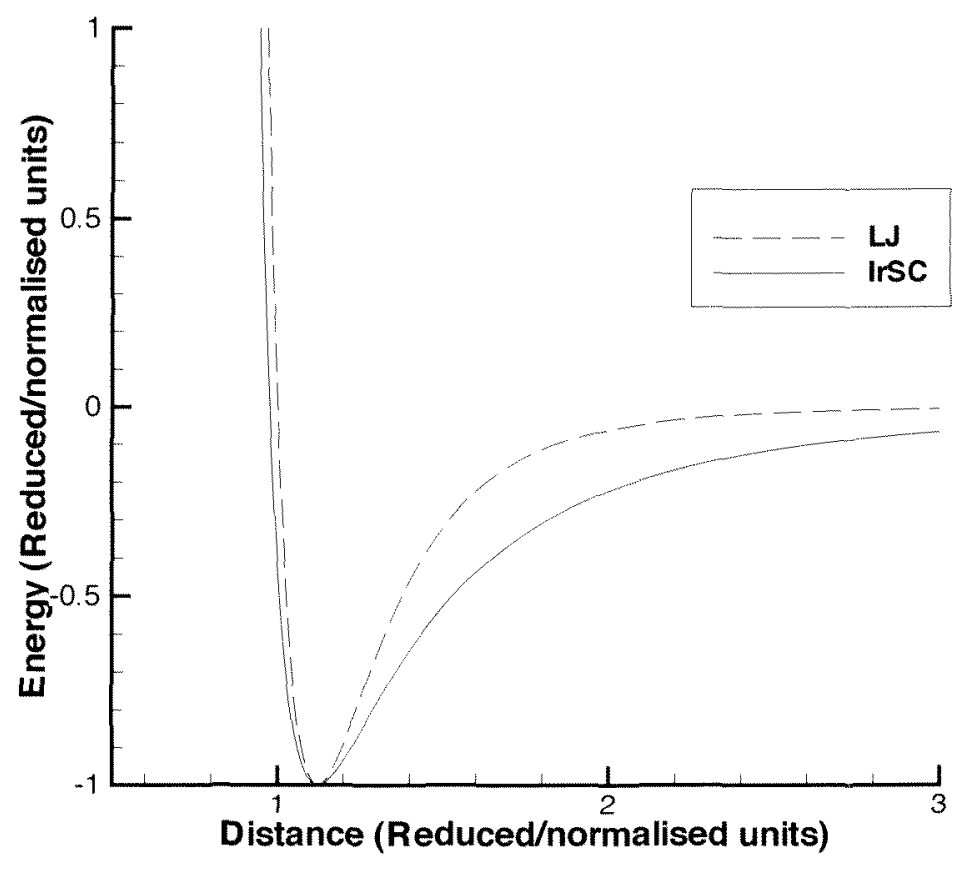

Figure 3.1: A comparison between the interaction energies for the LJ and IrSC potentials for a two-body interaction. For the LJ potential we are using $\sigma=1$ and $\epsilon=1$ and we have normalised the IrSC potential to set the well depth and equilibrium distance to $2^{\frac{1}{6}}$. With both potential wells normalised to the same depth, LJ has a noticeably shorter ranged attractive component. 


$$
E_{\text {total }}=\left[\frac{1}{2} \sum_{i} \sum_{j \neq i} V\left(r_{i j}\right)-\sum_{i} \phi\left(\rho_{i}\right)\right] .
$$

EAM potentials split the energy into two parts, a two body term $V$ and a many body term $\phi$. The two-body term is a repulsive core-core potential. The many body term comes from concepts in density-functional theory [45]. The basic idea is that the energy to place an atom at a site is purely determined by the electron density at that site, and not on the source of the electron density [46]. This particularly makes sense in metals, where atoms are embedded in a "sea" of valence electrons that are not strongly bound to any particular atom. The Sutton-Chen forms for these terms are

$$
\begin{gathered}
\phi\left(\rho_{i}\right)=\epsilon c \sqrt{\rho_{i}} \\
\rho_{i}=\sum_{j \neq i}\left(\frac{a}{r_{i j}}\right)^{m}
\end{gathered}
$$

and

$$
V\left(r_{i j}\right)=\epsilon\left(\frac{a}{r_{i j}}\right)^{n}
$$

where $m, n, \epsilon, c$ are fitting parameters that are particular to each material. We used the values for Iridium given in [43] for bulk Iridium, which are $m=6, n=14, \epsilon=2.4489 \times 10^{-3}, c=334.94$. See Table 3.1 for a comparison of the properties of Sutton-Chen Iridium with experimental Iridium. The agreement is decent for such a simple potential, but a more sophisticated potential could be much more precise. We used a cut-off distance of $20 \AA$, which was determined by increasing it until the barrier energy converged within the precision of the NEB algorithm.

\subsubsection{Relaxation}

To relax the images we used the FIRE algorithm [26] as described in section 2.2.1. Our results converged reasonably quickly with the parameters set to $N_{\text {min }}=5, f_{\text {inc }}=1.1, f_{\text {dec }}=0.5 . \alpha_{\text {start }}=0.1$ and $f_{\alpha}=0.99$, the values 


\begin{tabular}{|rr|r|l|}
\hline & & IrSC & Experimental \\
\hline Bulk modulus $B^{f}\left(\mathrm{eV}^{-3}\right)$ & & 2.28 & 2.31 \\
Elastic constants $\left({\left.\mathrm{eV} \AA^{-3}\right)}^{c_{11}}\right.$ & 2.97 & 3.74 \\
& $c_{12}$ & 1.94 & 1.60 \\
& $c_{44}$ & 1.32 & 1.68 \\
Cohesive energy per atom $(\mathrm{eV})$ & & 6.94 & 6.94 \\
Lattice parameter $(\AA)$ & & 3.84 & 3.84 \\
\hline
\end{tabular}

Table 3.1: Comparison of IrSC properties with experimental Ir properties. The lattice parameter $a^{f}$ and cohesive energy per atom $\left(E_{c}^{f}\right)$ are fitted exactly. Values taken from [43].

suggested in [26]. We set $\Delta t_{\max }=10^{-2}$ and the initial time-step $\Delta t=$ $10^{-3}$ in Lennard-Jones (LJ) time units. Varying these parameters did not noticeably alter the calculated barrier energy, but only affected the time taken to reach the minimum.

The FIRE algorithm is easy to implement within existing molecular dynamics code. We found it converges faster than simulated annealing, and is very robust.

\subsubsection{Nudged Elastic Band}

The NEB calculations were performed on hemispherical clusters, $\sim 13$ atoms in diameter as shown in Fig. 3.2. Different clusters were produced for each type of hop, as well as for each combination of neighbours in each hop, as described in section 3.2.2. The hopping atom and the relevant combination of neighbours were placed on the top (the flat surface) of the cluster. Barriers were determined for several thousand different types of hop including many permutations of nearest neighbour positions. We found it was necessary to add a shell of atoms around the atom's neighbours, as otherwise these neighbour atoms were often in unstable positions and would lose their configuration during the initial relaxation. Any atom that 

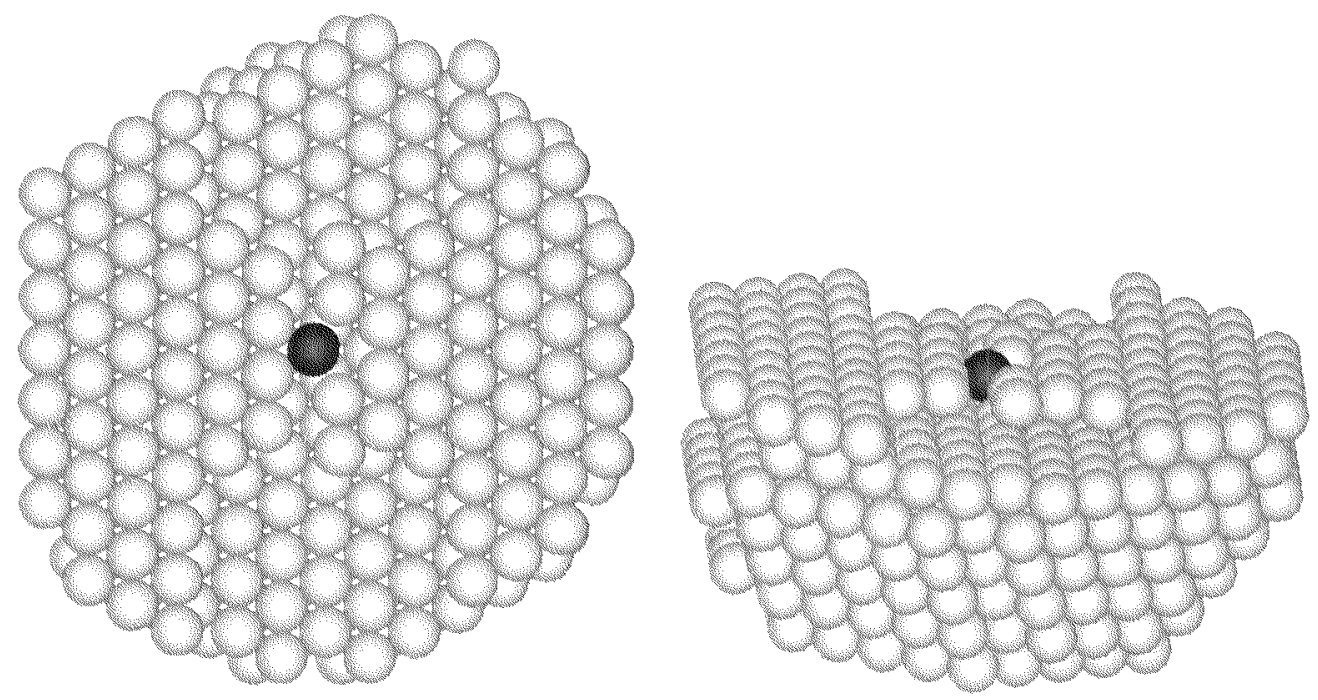

Figure 3.2: An example of the initial configuration used for NEB calculations (view from above and from side). The moving atom is marked as black, and in this instance moves one site to the right, from having two neighbours in the (111) plane to having four.

was too far away from the moving atom was frozen (i.e. $v_{a t o m}=0$ ) to retain the structure. The distance was $4 \sigma$ for LJ and $12 \AA$ for IrSC.

The initial and final states were relaxed using FIRE first for 12000 iterations, without the NEB force, to converge on stable start/end points. Then the final and initial states were fixed and the in-between states were relaxed with the NEB force. The climbing image was activated when the average of the total sum of the forces over all moveable atoms in each moveable image was less than $10^{-3}$ in LJ force units (or the equivalent in real units) or after 1600 iterations, whichever came first, and the final barrier energy was outputted when the summed force was less than $10^{-4}$ in LJ force units (or the equivalent in real units) or after 8000 iterations, whichever came first. We examined the force convergence rather than the energy barrier convergence because the force converged more slowly.

For a number of hops, either the initial or final configuration was found 
to be unstable. During the initial relaxation phase, the unstable endpoint would fall into the other endpoint. Two examples are shown in Fig 3.3. We interpreted this as an "instantaneous move". That is, any atom that moves into the unstable position immediately performs the move, and any atom that attempts to move from the stable position to the unstable position is prohibited from doing so. Hence the move was given a very large rate to simulate an "instantaneous move" that would give a time-step of effectively zero, when we take into account rounding errors. The reverse move was given a rate of 0 - that is, the reverse move was impossible as it was not moving into a stable site.

\subsubsection{Barrier Calculations}

Using the NEB method, as expected we found the barrier energy of a hop increased with the number of initial neighbours (on the same (111) plane). Where $N_{f}$ and $N_{i}$ are the number of final and initial neighbours respectively, and with the LJ potential, a fit to the data (Fig. 3.4) gives $E_{b}=0.21 \epsilon N_{i}+0.18 \epsilon$. A plot of barrier energy against number of final neighbours (Also Fig. 3.4) has a shallower slope $\left(E_{b}=-0.11 \epsilon N_{f}+0.71 \epsilon\right)$.

Similarly for the IrSC potential, fitting the data (Fig. 3.5) gives us $E_{b}=$ $0.13 N_{i}+0.11$ (in eV) and $E_{b}=-0.088 N_{f}+0.48$ (in eV). Some KMC studies [33] use a model where the barrier energy of a hop is proportional to the number of initial neighbours the atom had. While our data confirms that initial neighbours are more important in determining the barrier energy than final neighbours, the non-zero intercepts suggest in our fits that a simple proportionality is not suitable. The wide spread suggests that using a look-up table rather than a formula based on number of initial or final neighbours would be a better approach.

For the LJ case, the barrier energy for an adatom diffusion step was determined to be $0.3135 \mathrm{e}$ for hops from HCP to FCC sites and $0.3109 \epsilon$ for hops from FCC to HCP sites. This agrees with Agrawal et al's [47] range 

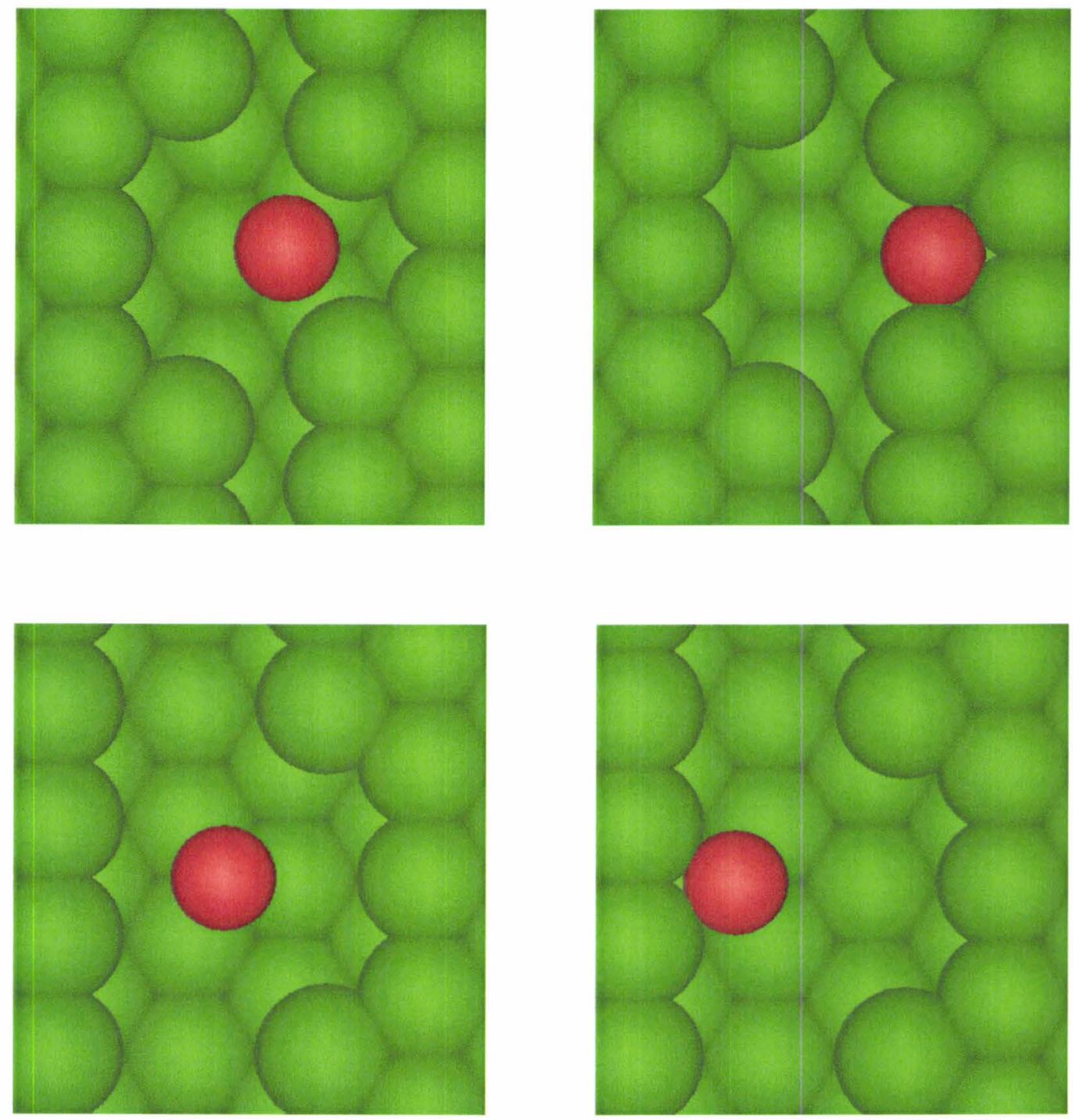

Figure 3.3: Top: In this hop, the initial state is unstable. The atom is easily pulled into the pocket. Bottom: The initial state is unstable as it initially has no neighbours. 

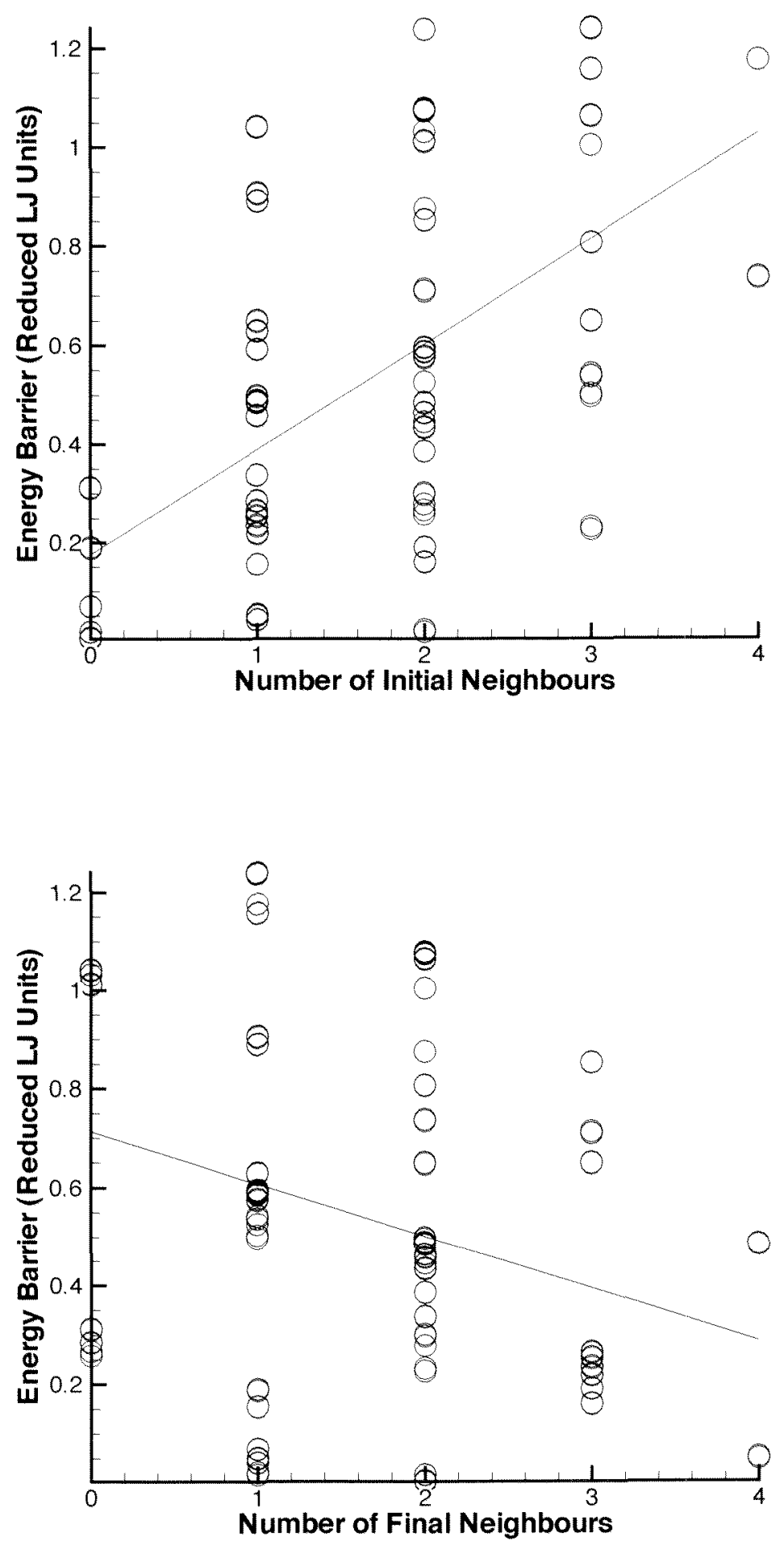

Figure 3.4: Above: A plot of initial neighbours versus barrier energy. Below: A plot of final neighbours versus barrier energy. There are obvious tendencies in each graph, but the fit is very poor. 

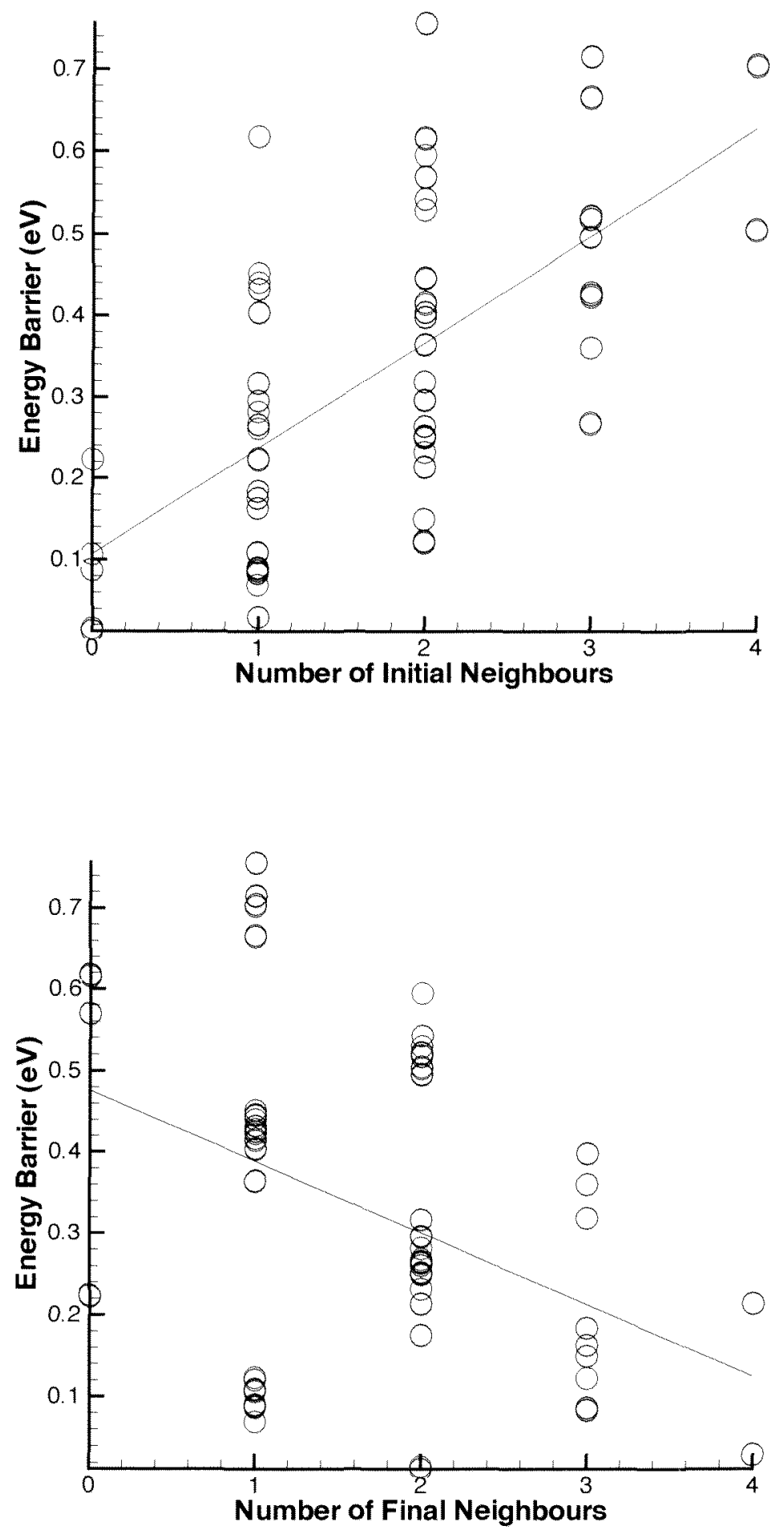

Figure 3.5: Above: A plot of initial neighbours versus barrier energy. Below: A plot of final neighbours versus barrier energy. As in the LJ case, there are obvious tendencies in each graph, but the fit is very poor. 
of $0.26 \epsilon-0.32 \epsilon$ using Monte-Carlo variational transition state theory and Zoontjens' [48] value of $0.305 \epsilon$ using a slightly different method of NEB calculations. We also confirmed the existence of the Ehrlich-Schwoebel barrier, with a hop off a ledge having a barrier energy of $1.460 \epsilon$, almost 5 times more than simple diffusion. For IrSC, the diffusion barriers were determined to be $0.2223 \mathrm{eV}$ for HCP to FCC and $0.2224 \mathrm{eV}$ for FCC to HCP, which is in agreement with Agrawal et al's value of $0.23 \mathrm{eV}$ for diffusion in LJ MD simulations, just outside the error range for the value of $0.27 \pm 0.4 \mathrm{eV}$ determined by field ion microscope observations [49], and under the value of $0.30 \pm 0.01 \mathrm{eV}$ from STM. The IrSC potential appears to underestimate the diffusion hop barrier energy. A hop off a ledge has a barrier energy of $0.8170 \mathrm{eV}$, almost 4 times more than simple diffusion, again confirming the existence of the Ehrlich-Schwoebel barrier.

The energies in the LJ potential are proportionally more strongly affected by changing the number of initial and final neighbours than the energies in the IrSC potential. For example, an LJ kink-flip hop has an energy barrier about 2 times as high as an LJ diffusion step, while an IrSC kink-flip hop has an energy barrier about 1.5 times as high as an IrSC diffusion step. This may be because IrSC is a longer ranged force, and so the potential energy of an atom is not as dramatically altered by atoms moving short distances.

The energy difference between hops from HCP and FCC sites is small, and is likely to be strongly affected by truncation errors. An LJ lattice sum of a slab of $10^{8}$ FCC atoms shows an HCP adatom has an energy $0.008 \mathrm{e}$ lower than an FCC adatom, which is only roughly in agreement with our difference in barrier energies between an HCP to FCC and an FCC to HCP hop $(0.0026 \epsilon)$. This suggests that our implementation of the NEB code does not accurately distinguish these small differences in energy. However, as we discuss below, a small difference in the barrier energies has a significant effect in the results of our KMC simulations, although the precise size of these small differences does not seem important for the qualitative 
evolution of the system.

Convergence tests (Fig. 3.6,3.7) confirm that the small differences are not a consequence of the cut-off distance, but other NEB kink-flip energy barrier calculations [48] with the LJ potential using a different initial slab set-up show HCP has a lower energy - the opposite to what we observed. Whether hops from HCP or FCC have lower barriers according to NEB calculations seems to be very sensitive to the configuration of atoms, even at some distance from the hopping atom. It appears that our NEB-based method can not determine energy barriers at the level of accuracy required to tell the difference between an HCP to FCC and an FCC to HCP hop with these potentials, so any preference we found should be interpreted with caution. The differences according to our potentials are of order $10^{-4} \mathrm{eV}$ or $10^{-4} \sigma$ while our NEB code is only consistent to about $10^{-2} \mathrm{eV}$ or $10^{-2} \sigma$.

A summary of the barrier energies for several significant hops is given in Fig. 3.2 .

\subsection{Kinetic Monte-Carlo}

\subsubsection{Lattice Implementation}

The lattice was parameterised by a three-dimensional mesh, which is stored as a Boolean array. Simulations of this sort typically use a refined lattice which allows both HCP and FCC sites [10, 12]. The mesh is parameterised by three basis vectors - two in the (111) plane separated by $60^{\circ}$, and a third orthogonal to the others - which gives layers of triangular meshes. Atoms are forbidden from being adjacent to or directly on top of another atom i.e. the radius of an atom is the length of one basis vector "step". Hence the nearest neighbour distance is two steps, as shown in Fig. 3.8.

The mesh is periodic in the lateral directions, but open in the vertical. The periodicity of the boundary made is necessary to use grain boundaries in our grain boundary propagation simulations, so that atoms that 


\begin{tabular}{|r|r|r|}
\hline Hop & LJ Barrier $(\epsilon)$ & IrSC Barrier (eV) \\
\hline Kink-flip to HCP & 0.6505 & 0.3602 \\
Kink-flip to FCC & 0.6490 & 0.3606 \\
Pocket-fill & 0.4847 & 0.2140 \\
Reverse pocket-fill & 0.7366 & 0.5042 \\
Diffusion step to HCP & 0.3135 & 0.2223 \\
Diffusion step to FCC & 0.3109 & 0.2224 \\
Pinch-plug (2 to 3) & 0.8522 & 0.3191 \\
Reverse pinch-plug (2 to 3) & 0.2329 & 0.2685 \\
Pinch-plug (1 to 3) & 0.2610 & 0.0853 \\
Reverse Pinch-plug (1 to 3) & 0.5232 & 0.4269 \\
\hline 2 to 3 pinch-plug forward & & \\
versus backward ratio & 3.6587 & 1.1884 \\
\hline 1 to 3 pinch-plug forward & & 0.1998 \\
\hline versus backward ratio & 0.4990 & \\
\hline Kink-flip barrier to HCP & & 0.9989 \\
\hline versus barrier to FCC ratio & 1.0023 & \\
\hline
\end{tabular}

Table 3.2: Important barrier energies. These hops are described in more detail in Section 4.1.1. For the pinch-plug hops, "2 to 3" and "1 to 3 " refer to the number of initial neighbours (1 or 2) and final neighbours (3) in the hop. Note that IrSC atoms are more likely to enter and remain inside a pinch site than LJ atoms. This data also shows the slight preference for FCC in LJ (as the barrier to HCP is larger) and the preference for HCP in IrSC. 


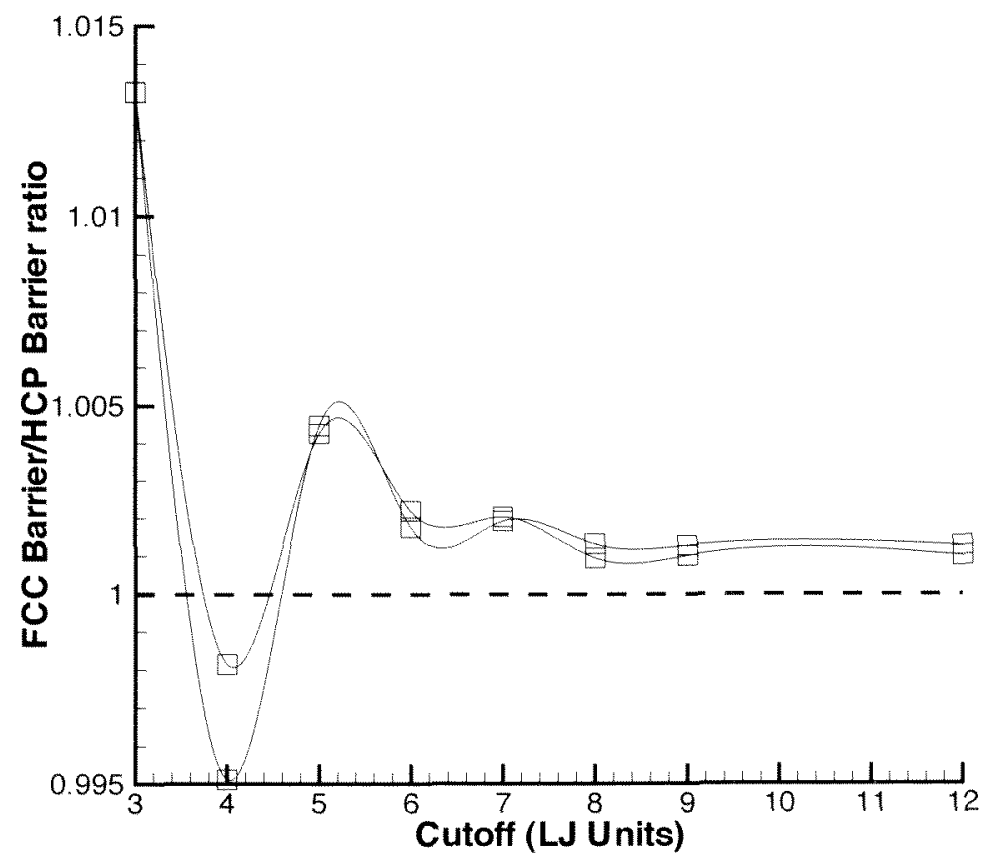

Figure 3.6: The ratio between the barrier energy of an FCC to HCP kinkflip hop and an HCP to FCC kink-flip hop as a function of cut-off distance for LJ. The second set of data points is the barrier energy from a calculation performed on same hop flipped across the axis of the hop. This should give the same barrier energy, so the difference between them is the uncertainty in our barrier. As it converges, the FCC to HCP hop has a slightly higher barrier than the HCP to FCC hop. The line is simply a spline to aid the eye. 


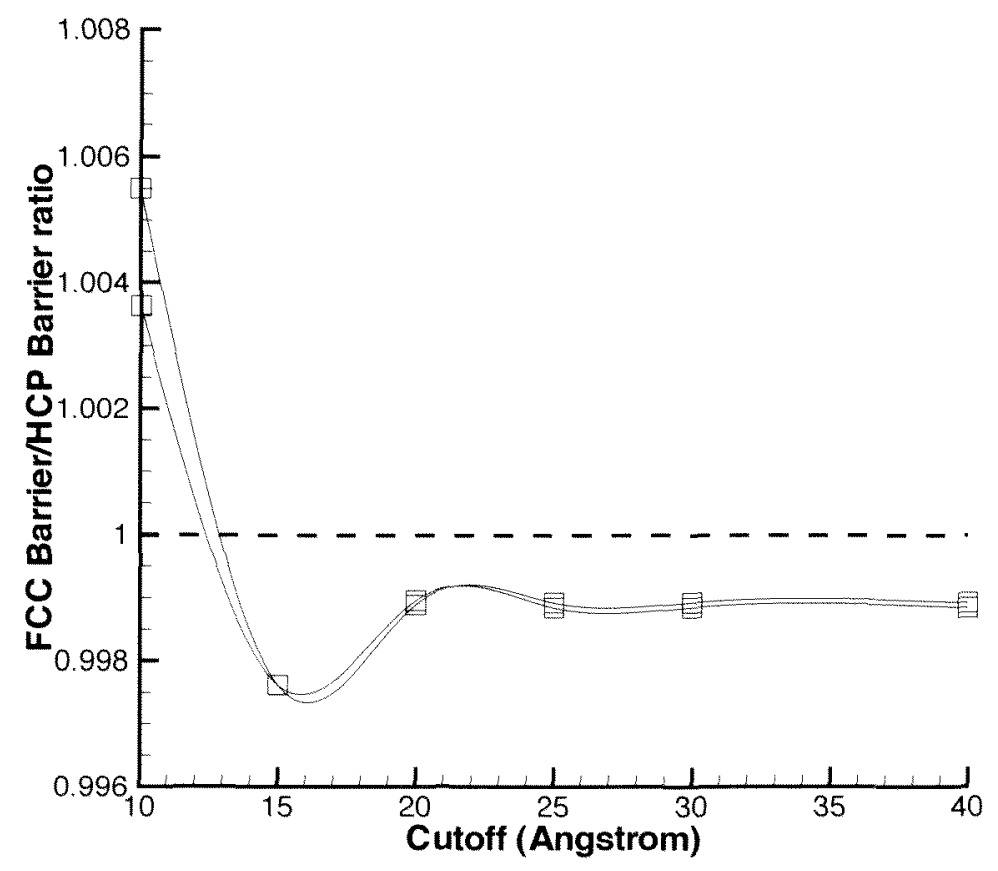

Figure 3.7: The ratio between the barrier energy of an FCC to HCP kinkflip hop and an HCP to FCC kink-flip hop as a function of cut-off distance for IrSC. The second set of data points is the barrier energy from a calculation performed on same hop flipped across the axis of the hop. This should give the same barrier energy, so the difference between them is the uncertainty in our barrier. As it converges, the HCP to FCC hop has a slightly higher barrier than the FCC to HCP hop. The line is simply a spline to aid the eye. 

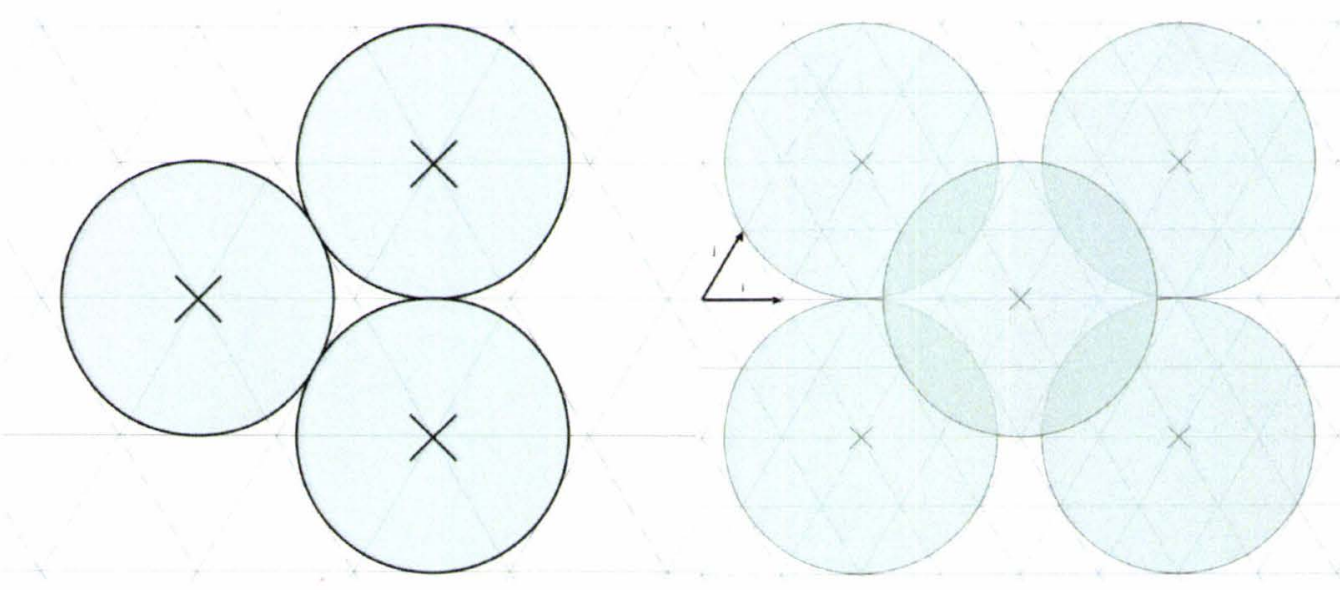

Figure 3.8: Left: The regular refined lattice mesh. Crosses mark the locations of atoms, and circles mark the excluded area around atoms. Right: The doubly refined lattice mesh. The central atom is one mesh level above the other atoms: it is in a decorated row site

were separated by a periodic boundary and hence were effectively adjacent were of the same stacking type. This meant we could not track grain boundary propagation over a long period of time, as the grain boundaries merge and stop propagating. The periodicity also effectively means we are simulating a grain boundary of infinite length. However, as noted in section 4.1.1, we found that the rate of grain boundary propagation decreases with the width of the periodic cell. This may be a cause for concern.

An atom in a decorated row site should be equidistant from the four supporting atoms (as our relaxation calculations confirm), but as the centre to centre distance across an A-gap is 3 steps and fractional steps are not permitted, we had to double the resolution of the mesh to allow atoms to enter decorated row sites, as shown in Fig. 3.8. This makes the nearest neighbour distance 4 steps. We called this mesh the "doubly refined lattice". As both coordinate systems were used, we refer to the low resolution method as the "singly refined lattice".

Hence the basis vectors are 


$$
\mathbf{i}=\left(\begin{array}{c}
0 \\
\frac{1}{\sqrt{6}} \\
0
\end{array}\right), \mathbf{j}=\left(\begin{array}{c}
\frac{1}{2 \sqrt{2}} \\
\frac{1}{2 \sqrt{6}} \\
0
\end{array}\right), \mathbf{k}=\left(\begin{array}{c}
0 \\
0 \\
\frac{2}{\sqrt{3}}
\end{array}\right)
$$

Every vector quoted from here uses these vectors as a basis, i.e. $\mathbf{i}=(1,0,0)$, $\mathbf{j}=(0,1,0)$ and $\mathbf{k}=(0,0,1)$. The vectors to an atom's nearest neighbours in the (111) plane are

$$
\left(\begin{array}{l}
2 \\
2 \\
0
\end{array}\right),\left(\begin{array}{c}
4 \\
-2 \\
0
\end{array}\right),\left(\begin{array}{c}
2 \\
-4 \\
0
\end{array}\right),\left(\begin{array}{c}
-2 \\
-2 \\
0
\end{array}\right),\left(\begin{array}{c}
-4 \\
2 \\
0
\end{array}\right),\left(\begin{array}{c}
-2 \\
4 \\
0
\end{array}\right) .
$$

Atoms are prohibited from being within 3 steps of another atom, where a "step" is any of these six vectors:

$$
\left(\begin{array}{c} 
\pm 1 \\
\mp 1 \\
0
\end{array}\right),\left(\begin{array}{c} 
\pm 1 \\
0 \\
0
\end{array}\right) \cdot\left(\begin{array}{c}
0 \\
\pm 1 \\
0
\end{array}\right)
$$

Also, all atoms must either be properly supported. If it is in an FCC or HCP site, it must be supported by three atoms out of

$$
\left(\begin{array}{c}
0 \\
-2 \\
-1
\end{array}\right),\left(\begin{array}{c}
-2 \\
0 \\
-1
\end{array}\right),\left(\begin{array}{c}
-2 \\
2 \\
-1
\end{array}\right),\left(\begin{array}{c}
0 \\
2 \\
-1
\end{array}\right),\left(\begin{array}{c}
2 \\
0 \\
-1
\end{array}\right),\left(\begin{array}{c}
2 \\
-2 \\
-1
\end{array}\right)
$$

If it is in a decorated row site, it must be supported by four atoms out of

$$
\left(\begin{array}{c}
2 \\
-3 \\
-1
\end{array}\right) \cdot\left(\begin{array}{c}
2 \\
1 \\
-1
\end{array}\right) \cdot\left(\begin{array}{c}
-2 \\
3 \\
-1
\end{array}\right) \cdot\left(\begin{array}{c}
-2 \\
-1 \\
-1
\end{array}\right)
$$

or

$$
\left(\begin{array}{c}
3 \\
-1 \\
-1
\end{array}\right) \cdot\left(\begin{array}{c}
-1 \\
3 \\
-1
\end{array}\right) \cdot\left(\begin{array}{c}
1 \\
-3 \\
-1
\end{array}\right) \cdot\left(\begin{array}{c}
-3 \\
1 \\
-1
\end{array}\right)
$$


or

$$
\left(\begin{array}{c}
1 \\
2 \\
-1
\end{array}\right),\left(\begin{array}{c}
-3 \\
2 \\
-1
\end{array}\right),\left(\begin{array}{c}
3 \\
-2 \\
-1
\end{array}\right),\left(\begin{array}{c}
-1 \\
-2 \\
-1
\end{array}\right)
$$

The matrix to rotate a vector by $60^{\circ}$ about the $\mathrm{k}$ direction is

$$
\left[\begin{array}{ccc}
1 & 1 & 0 \\
-1 & 0 & 0 \\
0 & 0 & 1
\end{array}\right]
$$

This can be used to calculate the hops in all six possible orientations given the hop in one direction.

Note that with this mesh, a mesh layer of size $L \times M$ can hold up to $\frac{L M}{12}$ close-packed atoms.

Several broad classes of hop were identified (illustrated in Fig. A.6 and on the mesh in Figs. 3.9-3.10). The principle hop is the "short hop", from an HCP to an FCC site or vice versa, given by some rotation of the vector $(2,0,0)$. The "decorated slide" is a longer hop, from a decorated row site to another decorated row site, along some rotation of $(2,2,0)$. Downwards hops of the form $(4,0,-1)$ are also permitted to allow atoms to hop down a terrace step. Upward hops (the reverse of the down hop, Fig. A.6, top right) are not permitted. Upward hops have large barriers because the atom moves from the side of a terrace, where it has at least 2 neighbours on the plane (i.e. the terrace wall) to sitting on top of the island, where it has at most 4 neighbours on the plane - the other two neighbouring positions can not be filled as they would have been resting on the atom that hopped. As well as generally reducing its number of neighbours (from $5-11$ to $3-7$ ), it must penetrate the Ehrlich-Schwoebel barrier. Hence using the IrSC potential at $300 \mathrm{~K}$ the fastest upward hop that we calculated was $\sim 10^{27}$ times slower than the fastest downward hop. By comparison, the fastest downward hop was $\sim 10^{17}$ times slower than adatom diffusion, one of the fastest processes. While downward hops were performed in our 
simulations, they were comparatively infrequent, and so we do not expect the even rarer upward hops to have a significant effect. Finally, hops of the form $(3,0,0)$ from an $\mathrm{HCP} / \mathrm{FCC}$ site into a decorated row site or vice versa are allowed. Hops to HCP sites were allowed to have different energy levels than hops to FCC sites.

Although an FCC to FCC or HCP to HCP "long hop" of the same form as the $(2,2,0)$ decorated slide is necessary to model some processes such as "hole" diffusion, these processes are much slower than the processes we are studying. We calculated the rate of a hole diffusion hop using the IrSC potential at $300 \mathrm{~K}$ is $\sim 10^{41}$ times slower than the kink-flip hop we describe later. By comparison, at $300 \mathrm{~K}$ the kink-flip hop is $\sim 10^{5}$ times slower than a diffusion hop. So the hole diffusion steps are $\sim 10^{46}$ times slower than a diffusion hop and it can be assumed that these moves do not happen on the time scale of our simulations. Also, most long hops are not valid hops for $\mathrm{KMC}$, as the atom moves through another stable site in the course of the hop - e.g. the atom moves from FCC to HCP to FCC. The barrier energy for these "double hops" is the same as the greatest barrier of the two individual hops - hence the double hop will have the same rate as a short hop, which will not correctly describe processes such as diffusion.

\subsubsection{The look-up table}

Each hop was parameterised with a twelve bit binary number according to its class, and the position of the moving atom's initial and final neighbours. The first three bits are set by the class of the hop (i.e. whether long, short, into or out of a decorated site etc), and the 4 th bit is set by whether the atom starts on an HCP or FCC site, although in the case of decorated sites this was ignored. Each of the initial and final neighbours has an unique ID from 0 to 7 , giving 8 bits to specify which neighbours are present, for a total of 256 different combinations, although for some hops (decorated slide, decorated entrance) not all 8 bits were assigned to a 

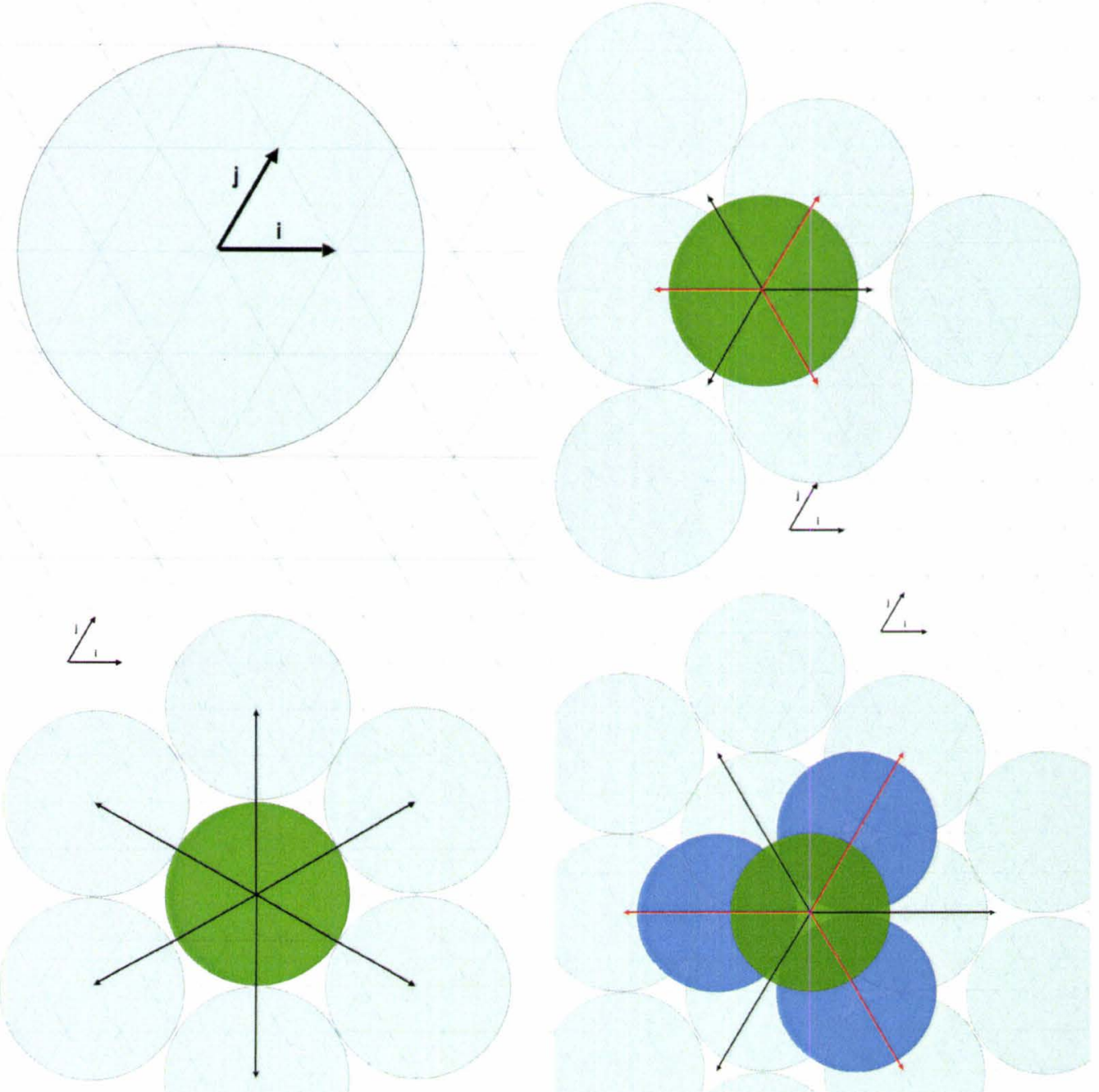

Figure 3.9: Top left: Unit vectors one mesh space long in the $\mathbf{i}$ and $\mathbf{j}$ directions, compared to the radius of an atom. Top right: The green atom is resting upon 3 light blue atoms. There are six "small hop" vectors. Moving the atom along any of the three black vectors would move the green atom into a valid site supported by three blue atoms, while moving the atom along the three red vectors would not. If the orientation of the supporting blue atoms was reversed, the orientation of valid and invalid short hops would also reverse. Bottom left: The vectors from the central green atom to its six nearest neighbours (light blue) on the same (111) plane. Bottom right: The green atom is resting on the dark blue atoms, which are resting on the light blue atoms. The vectors indicate "down hops", where the green atom would move down to the same level as the dark blue atoms. Hops on to sites that are occupied or not supported by three atoms are invalid - e.g. the three red vectors shown here. 

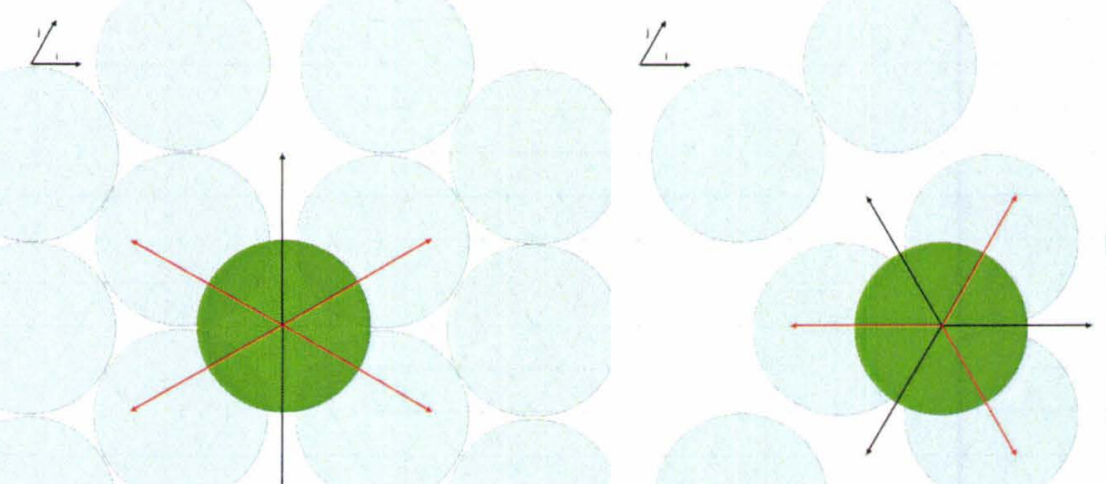

Figure 3.10: Left: The green atom is resting on four light blue atoms, in a 4-fold site along an A gap. The vectors indicate "decorated slide" hops, from one decorated site to another. Six different vectors are available, but in each orientation of the A gap only two vectors (black) move the atom onto a valid site. Red vectors are invalid hops. Right: The green atoms is resting on three blue atoms. The vectors indicate "decorated entrance" hops, from an HCP or FCC site to a decorated site. The hop is only valid if it is a hop on to a valid four-fold site (black vectors). These hops are reversible to allow atoms to hop from a decorated site onto an HCP / FCC site. Red vectors are invalid hops. 


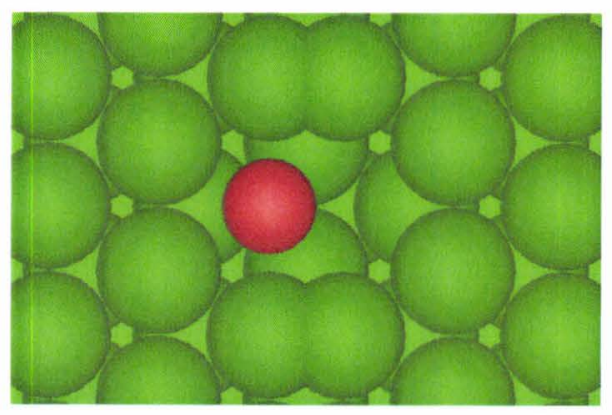

Figure 3.11: When all initial and final neighbours are switched on in a short hop NEB calculation, some of the initial and final neighbours overlap and are unstable. This situation is not permitted by our mesh rules, so the barrier energies for hops starting from this position were not calculated.

neighbour. Which atoms are considered neighbours depends on the type of initial and final sites (see Fig. A.6). We generated lattices with all combinations of final and initial neighbours for each hop class, and ran the NEB code to determine the energy barrier for each hop in the class. In this way we built up a 12 bit look-up table for the energy barriers for a large number of different types of hop. This would give $2^{12}=4096$ possible different hops, but the actual value is somewhat lower as not all classes of hop considered 8 neighbours, and also because for certain combinations of bits, the neighbours of the initial site overlap with the neighbours of the final site (as shown in Fig. 3.11) so these situations are unphysical and were not calculated.

To find the energy barrier of a particular hop during the KLMC routine, these 12 bits need to be determined. The first 4 are set by the type of hop and the type of sites involved (Fig. 3.3). The other 8 bits are set by the neighbours (Fig. A.6), rotated to correspond with the orientation used in the NEB calculation.

This method is more sophisticated than simple bond-counting methods. However, we observed that changing the geometry of the system to a flat rectangular slab while retaining the same neighbour configuration 


\begin{tabular}{|l|l|}
\hline Hop Type & Binary Code \\
\hline Short hop from FCC & 0000 \\
Short hop from HCP & 0001 \\
Decorated slide & 0010 or 0011 \\
Down hop from FCC & 0100 \\
Down hop from HCP & 0101 \\
Up hop from FCC (unused) & 0110 \\
Up hop from HCP (unused) & 0111 \\
Decorated entrance from FCC & 1000 \\
Decorated entrance from HCP & 1001 \\
Decorated exit to FCC & 1010 \\
Decorated exit to HCP & 1011 \\
\hline
\end{tabular}

Table 3.3: Parameterisation of classes of hop. The first three bits indicate the class of hop, the fourth bit indicates whether the hop is from an HCP or FCC site in most cases, except for the case of the hop out of a decorated site (a "decorated exit"), where it indicates where the hop is to an HCP of FCC site, and the decorated slide, where it is irrelevant. 
altered the barrier energies by as much as $\sim 0.01 \sigma$, suggesting that barrier energies of hops appear to be affected by atoms beyond their closest neighbours. This effect is small, but is critical for determining subtle effects such as a preference for $\mathrm{HCP}$ or $\mathrm{FCC}$, so in the future it would be desirable to develop an even more sophisticated method involving more atoms which would be more accurate.

\subsubsection{Data structures and algorithms}

The lattice is represented by an array of Boolean ( 1 bit logical) variables. The possible moves for each iteration are stored in a collection of arrays. Each array corresponds to a particular rate, of which we have calculated several thousand. Each entry in the array contains the position of the atom to be moved, and the position it would move to. This arrangement allows us to implement the Schulze [41] algorithm for choosing the hop, which is as follows:

1. Compute the overall rate $S_{N}=\sum_{i=1}^{N} r_{i} c_{i}$ where $N$ is the total number of rates, and $c_{i}$ is the number of events with rate $r_{i}$, together with partial sums $S_{i}=\sum_{j=1}^{i} r_{i} c_{i}$.

2. Select a random number $a \in\left[0, S_{N}\right)$

3. Search through the list of partial sums $S_{i}$ until $a<S_{i}$

4. Select an event from the set of events that occur at this rate by computing

$$
b=\left\lfloor\frac{S_{i}-r}{r_{i}}\right\rfloor+1
$$

5. Execute the event and update the configuration

This is a much faster method than performing a search through every atom or every hop, and is an $O(1)$ algorithm. This means the execution 
time for a hop does not scale with system size, although in practice, there is a slight decrease in speed with lattice size due to increase memory usage.

It is not necessary to loop over every atom to determine the list of possible hops every iteration as only the atoms close to the one that just moved would have their possible hops changed. So each iteration we only loop over the largest region that could have been affected by the hop $( \pm 12, \pm 12, \pm 2)$. First, however, the previous hops from this region have to be removed from the table of hops, and to do this efficiently (i.e. without a linear search through every hop), each lattice point needs to keep track of where in the hop arrays the hops that come from that lattice point are located. This essentially means there is some doubling up of data - both the lattice and the hop list "know" which hop goes from which location to which location. This data structure uses extra memory, and some extra computational overhead, but we found the increase in speed to be worth it. Except for the first time-step, where all hops must be calculated, we found the time taken for $10^{5}$ adatom hops to be almost constant as we increased the size of the lattice (Fig. 3.12), as expected for an $O(1)$ algorithm. By comparison, checking over every mesh point and rebuilding the entire move list each time for a 48 by 48 mesh took about three times as long as our fast algorithm, and the time taken per iteration increased roughly linearly with $N$, the number of mesh points, instead of remaining constant, giving significant slowdown for larger meshes.

\subsubsection{Other details}

We have represented deposition as another KMC process, with a rate equal to the deposition rate in atoms/time unit. When a deposition event is chosen by the KMC algorithm, all possible landing sites are determined and one is randomly selected. We have assumed that all landing sites have the same probability of receiving an atom. We have also chosen to neglect evaporation, so once an atom has landed on the surface, it remains 


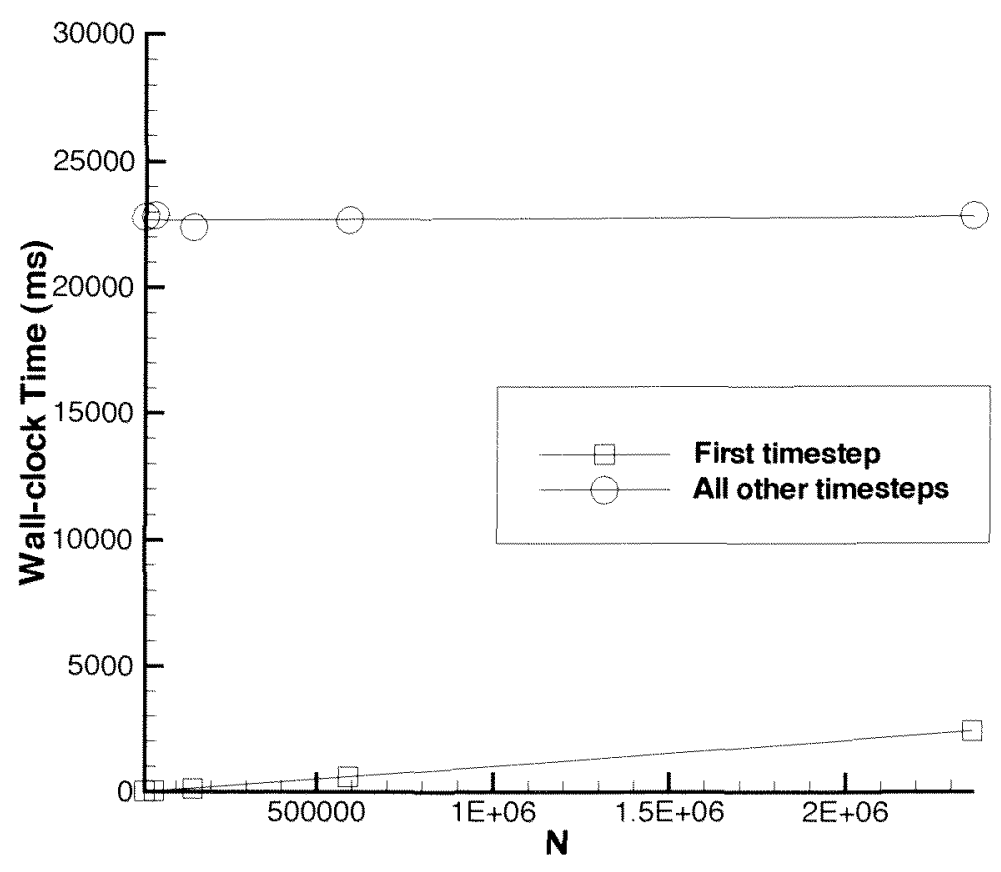

Figure 3.12: Time taken for $10^{5}$ diffusion hops in milliseconds as a function of lattice size compared to time taken for the first time-step. The first timestep increases with the mesh size, as every hop needs to be calculated initially. The time taken for the remaining steps is roughly constant, as the region that is looped over is independent of the lattice size. The mesh had $N$ points, four layers high with each layer being "square" i.e. $L$ by $L$, where $L=\sqrt{N / 3}$. The lattice of atoms filled 3 of these four layers with FCC. The fourth layer contained $\frac{5 L}{48}$ adatoms. 
there. The condition for this to be a valid assumption is for the evaporation barrier energy to be much larger than the barrier energies of the important processes in the substrate. With NEB in LJ we calculated evaporation to have a barrier of $1.55 \epsilon$, which is larger than the critical hops.

We arbitrarily set the pre-factor mentioned in Eq. 2.19 to $10^{7}$, simply to ensure the numbers in the algorithm were large enough that round-off errors would not be a problem. This was divided out when data such as diffusion coefficients were extracted from the program, and later the real pre-factor is calculated.

\subsection{Adatom diffusion and determining pre-factors}

We placed a single adatom on an FCC substrate, and observed its position as a function of time as it diffused over the surface for 100 KMC steps. This was repeated 1000 times and the results averaged in order to find the diffusion coefficient of an adatom on a (111) FCC surface at various temperatures, from the 2-D diffusion formula

$$
<x^{2}>=4 D t
$$

The diffusion coefficient is related to temperature by the formula

$$
D^{*}=D_{0}^{*} \exp \left(\frac{-E_{b}^{*}}{T^{*}}\right)
$$

where $D^{*}$ is the diffusion coefficient at a particular temperature, $D_{0}^{*}$ is the limit of the diffusion coefficient as temperature goes to infinity, $E_{b}^{*}$ is the energy barrier of a diffusion step and $T^{*}$ is the temperature. The star indicates these are in LJ reduced units, e.g.. $T^{*}=\frac{k_{b} T}{\epsilon}$ where $k_{b}$ is Boltzmann's constant and $T$ is the absolute temperature. Plotting $\ln \left(D^{*}\right)$ versus $\frac{1}{T^{*}}$ gives us $D_{0}=0.3098$ and $E_{b}=0.3122 \epsilon$ (Fig. 3.13). The calculated energy barrier is close to the average of the energy barriers for HCP to FCC and FCC to HCP diffusion steps from NEB - $0.3135 \epsilon$ and $0.3109 \epsilon$ - as we should expect. However, $D_{0}$ differs from the value of $D_{0}=0.077$ given by Agrawal 


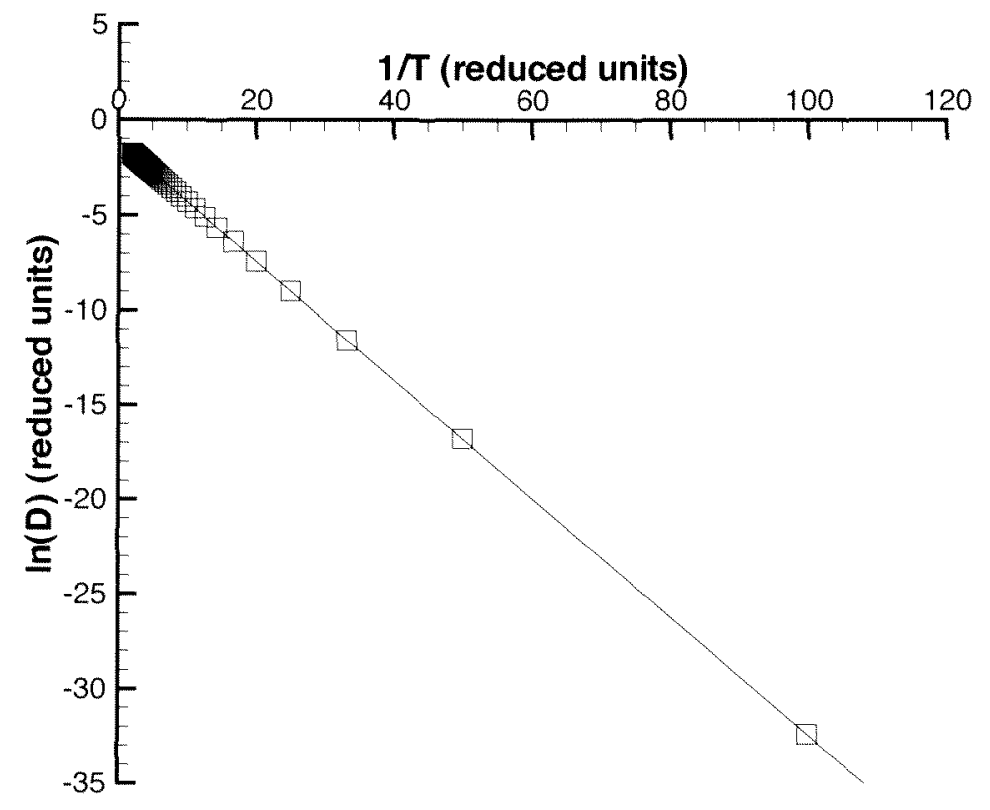

Figure 3.13: Plotting the $\log$ of diffusion coefficient against $1 / T^{*}$ to determine $D_{0}$ for LJ. $D$ is in reduced LJ units. The fit is $\ln \left(D^{*}\right)=\frac{-0.3122 \epsilon}{T^{*}}-1.1718$ (in LJ units).

et al's calculations using Monte Carlo variational transition state theory and the Lennard-Jones (L-J) interactions [47]. This is because we haven't taken into account the pre-factor (effectively the "attempt frequency") in the KMC rate formula - essentially, after dividing out the arbitrary $10^{7}$ factor, the pre-factor is 1 . Hence we can use the ratio between our $D_{0}$ and the value given in Agrawal et al to determine our pre-factor for LJ, which comes to 0.249 .

To match our simulated results with experiment, we must convert the LJ reduced units into "real" units. If we take the LJ material as being Iridium, we can use the values of $\sigma=2.419 \AA$ and $\epsilon=0.830 \mathrm{eV}$ from [47] for 


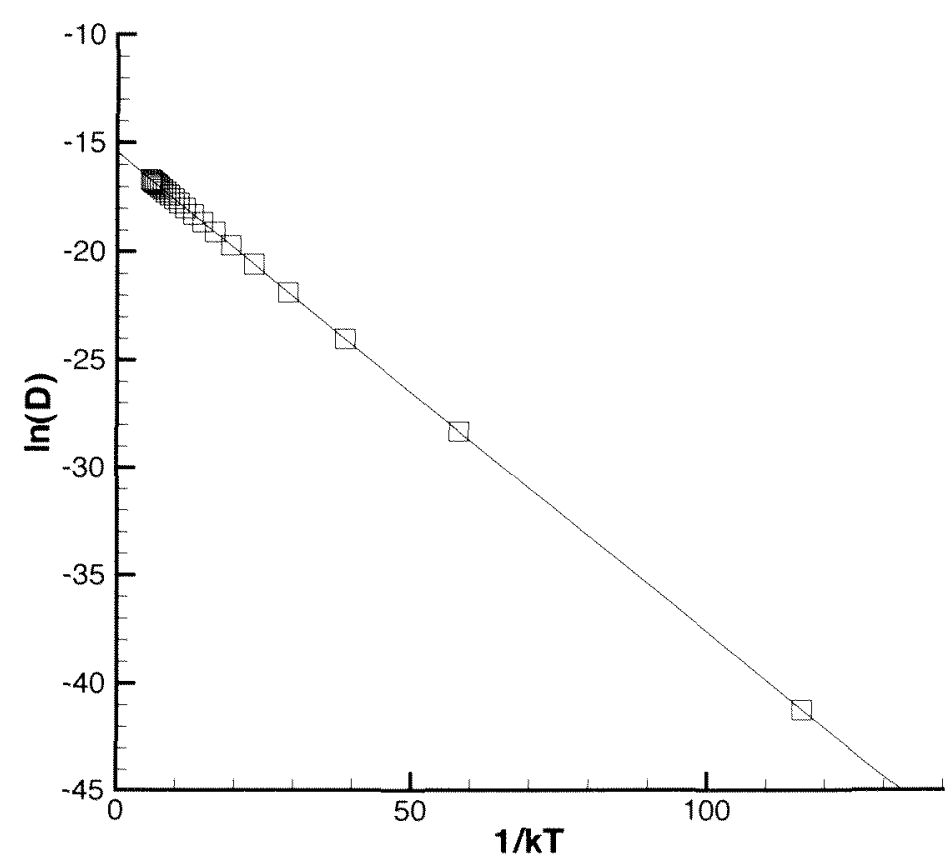

Figure 3.14: Plotting the $\log$ of diffusion coefficient against $1 / k_{b} T$ to determine $D_{0}$ for Iridium. $D$ is in units of $\mathrm{m}^{2} / \mathrm{s}$. The fit is $\ln (D)=\frac{-0.2224}{k_{b} T}-15.406$ (in real units). As in the $L J$ case, the barrier energy is unsurprisingly in good agreement with our NEB-calculated diffusion step energies $(0.2223$ and $0.2224 \mathrm{eV})$. 
our distance and energy scales. Then we can use the formula

$$
\tau=\sqrt{\frac{m \sigma^{2}}{\epsilon}}
$$

from dimensional analysis to calculate the time scale, where $\sigma$ and $\epsilon$ and the LJ length and energy scales and $m=192.217 \mathrm{amu}$ is the mass of Iridium [50]. The result is $\tau=3.75 \times 10^{-13} \mathrm{~s}$. This gives the pre-factor in real units as $6.6 \times 10^{11} \mathrm{~Hz}$.

Distance and energy are already in real units in the IrSC potential, so the diffusion Arrhenius formula is now

$$
D=D_{0} \exp \left(\frac{-E_{b}}{k_{b} T}\right)
$$

and only the pre-factor needs to be determined to determine the correct time-scale. If we assume a pre-factor of $1 \mathrm{~Hz}$, our $\mathrm{KMC}$ calculations give a diffusion constant of $D_{0}=2.8 \times 10^{-16} \mathrm{~cm}^{2} / \mathrm{s}$. The STM measurements in [51] gives $D_{0}=0.00092 \mathrm{~cm}^{2} / \mathrm{s}$. This gives a correct pre-factor of $3.26 \times 10^{12}$ $\mathrm{Hz}$. 


\section{Chapter 4}

\section{Results and Analysis}

Using the KMC method described in the previous chapter, we performed simulations of grain boundary propagation and film growth by deposition. In grain boundary simulations, we observed that a small energy difference between the barrier energies of HCP to FCC and FCC to HCP kink-flips caused one stacking type to quickly take over the entire slab. We also observed that when grain boundaries merge under the IrSC potential, they are more likely to form a series of "lakes" and "bridges" rather than a long channel, as is the case for the LJ potential.

The deposition experiments grew triangular islands, as observed in experiment. However, limited processor time forced us to set the deposition rate quite high, which caused islands to grow layers of terraces on top, rather than remaining flat. These terraces prevented self-healing of the layer.

Section 4.1 gives the results for the grain boundary propagation simulations. Subsection 4.1.1 gives the results for a simulation of two parallel periodic grain boundaries of infinite length, including details on atomistic processes involved in propagating the grain boundary, and the merger of grain boundaries. Subsection 4.1.2 gives the observations on a grain boundary enclosing a region. Here, the grain boundary shrunk to transfer all the enclosed region to the opposite stacking type. Subsection 4.1.3 ex- 
plains the effect of adatoms on the surface, including their formation into island nuclei, their effect on the grain boundary, and the role of decorated sites.

Section 4.2 gives the results for deposition simulations, including the island shape and terracing.

When not explicitly specified, results using units of $\mathrm{eV}$ and $\AA$ are from simulations using the IrSC potential, while results using units of $\sigma$ and $\epsilon$ are from simulations using the LJ potential.

\subsection{Grain Boundary Propagation}

\subsubsection{Propagation of Flat Boundaries}

Inspired by Zoontjens et al [25], for the simplest grain boundary case we set up a rectangular slab, 3 layers high, with the middle half of the upper layer HCP, and the outer quarters FCC. "Flat" grain boundaries lie between these regions. When we replaced the flat grain boundaries with chevrons (Fig. 4.12, bottom right), we found that very quickly the boundaries lost their chevron shape (see Fig. 4.2, top) and continued to evolve along the same lines as the flat boundary so we conclude that we can run simulations with only a flat boundary without loss of generality. The bottom two layers were fixed and so was essentially a 2D simulation (Fig. 4.1). A large number of simulations were performed, over which the dimensions of the mesh ranged from $48 \times 48$ up to $192 \times 96$. This range of mesh sizes corresponds to a range from 192 to 1536 atoms per complete FCC or HCP layer. Incomplete layers (such as top layers with grain boundaries) have fewer atoms. The temperature in these runs varied from about $0.5 \epsilon-1.06$ for the LJ runs, or $300 \mathrm{~K}-1000 \mathrm{~K}$ in the IrSC runs. We noticed no major difference resulted from changing the temperature through these ranges, other than the grain boundary's diffusion coefficient increasing with temperature, as the rates of the hops increased according to the 


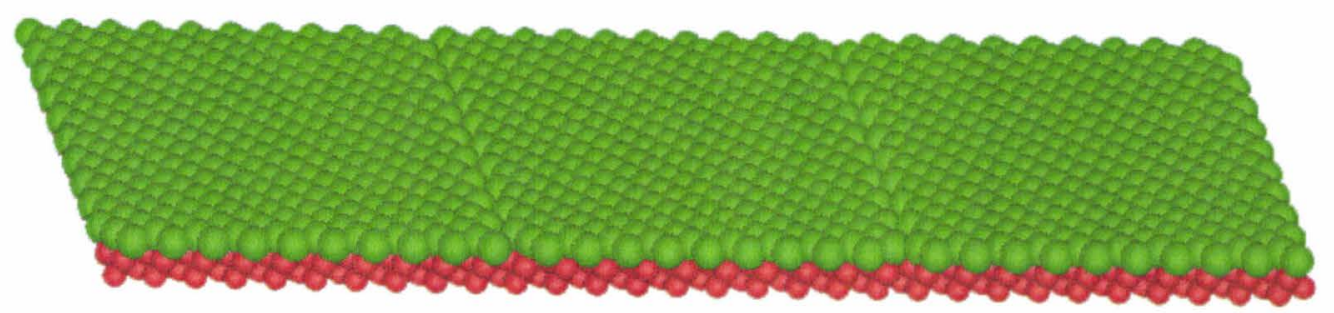

Figure 4.1: An example of the set-up for grain boundary propagation simulations. Mobile atoms are on the top level, and are shown as larger and lighter here.

Arrhenius equation.

It was observed in LJ material that the grain boundaries had a very strong tendency to move towards the HCP side - that is, FCC dominated, while with IrSC, HCP dominated. As there were two grain boundaries, they merged to form a channel or series of gaps (see Fig. 4.4). To test the strength of the FCC/HCP preference, we ran 100 simulation runs on large and small slabs (Fig 4.3). For LJ, FCC dominated every time. In IrSC, HCP was more dominant than FCC, but at the smaller mesh sizes this dominance was not as strong - the final state of all atoms in the top layer was HCP only $66 \%$ of the time for a $48 x 48$ mesh. This is consistent with grain boundary propagation being a biased 1D random walk. Every iteration, the average position of a grain boundary can move slightly into the HCP region or slightly into the FCC region. The direction of the move is random but with a bias favouring the growth of FCC for LJ or HCP for IrSC. However, the random fluctuations are large enough that when the distance between grain boundaries is small, they can merge on the "wrong" side. If the distance between grain boundaries is sufficiently large, the bias is much more dominant and the system acts consistently. For LJ however, the bias was so strong that these fluctuations were too small to have an effect.

If the grain boundaries propagate like a random walk, their movement 

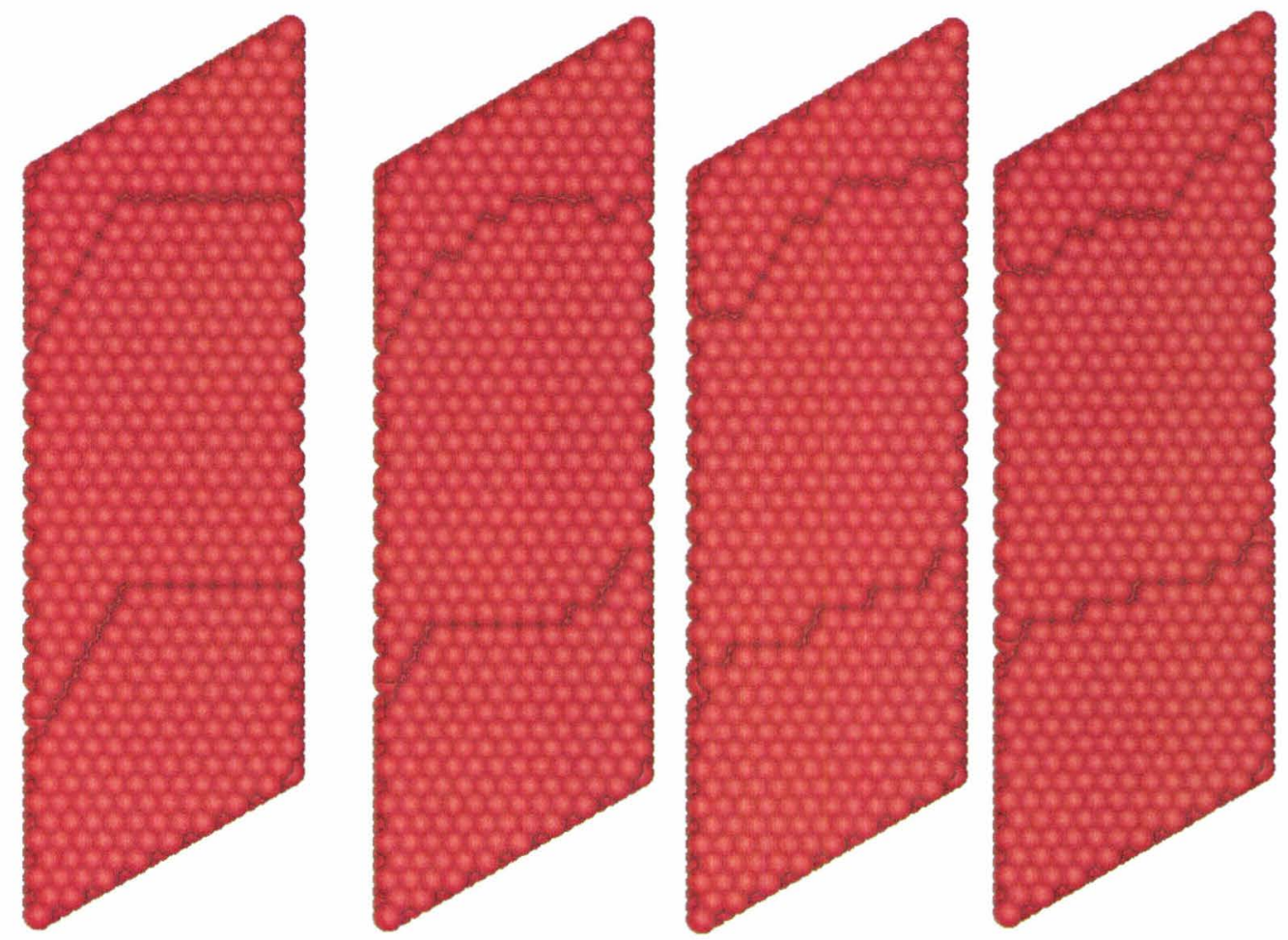

Figure 4.2: A chevron grain boundary quickly collapes into a zig-zag shape. The centre region is HCP and the outer region is FCC. 


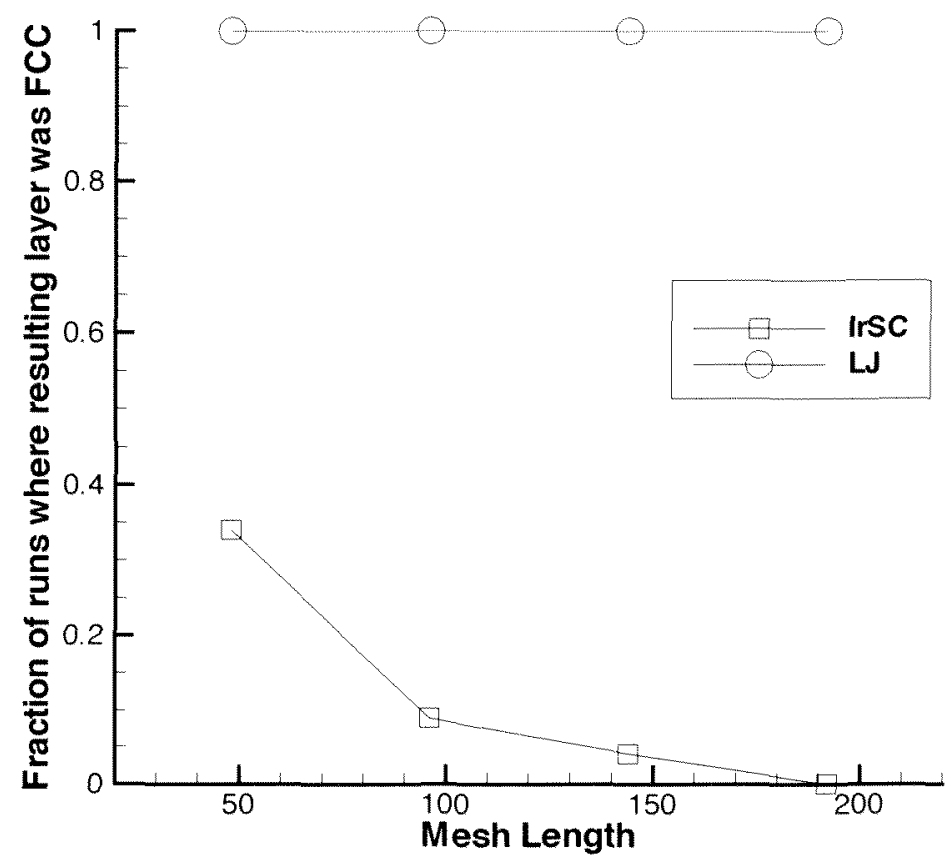

Figure 4.3: Fraction of runs where the entire layer healed to FCC as a function of the space between grain boundaries. Squares are IrSC data, circles are LJ data. The initial spacing between grain boundaries is half the mesh length. 


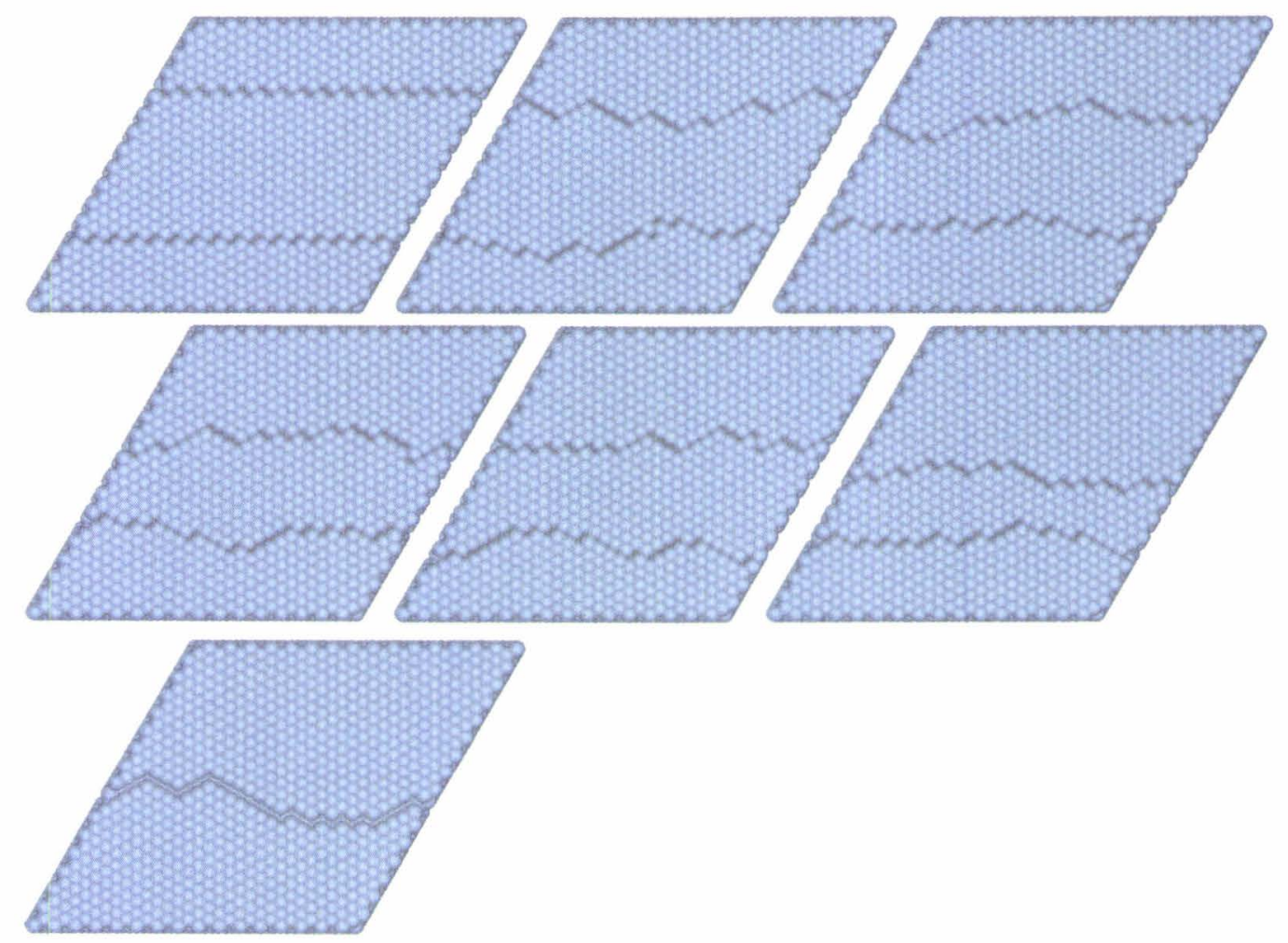

Figure 4.4: Snapshots equally spaced in simulation time of the merger of two grain boundaries into a channel, using rates taken with the LJ potential 
should obey the $1 \mathrm{D}$ diffusion law,

$$
<x^{2}>=2 D t
$$

where $\left\langle x^{2}\right\rangle$ is the mean-squared-distance the grain boundary has moved, $t$ is the time elapsed and $D$ is a diffusion coefficient. The mean is an average of a very large number of grain boundary simulations. If the number of simulations is large enough, this will converge even with a fairly short simulation time. This diffusion coefficient should follow the Arrhenius formula,

$$
D_{0}=D \exp \left(\frac{E_{b}}{k_{b} T}\right) .
$$

We made Arrhenius plots of the relationship of the diffusion coefficient with temperature and confirmed this relationship (Fig. 4.5, 4.6). The fits give the barrier energies for the most important hops in grain boundary propagation: $E_{b}=0.68 \epsilon$ for $\mathrm{LJ}$ and $E_{b}=0.37 \mathrm{eV}$ for IrSC. These are slightly above the values for kink-flip hops $(0.65 \epsilon$ and $0.36 \mathrm{eV})$, which suggests that the kink-flip hops are dominant, but not the sole hop involved in grain boundary propagation. We confirmed this by tracking hops in a dual grain boundary simulation. Out of 32000 hops, 23210 were kink-flips, and 8790 were "pocket-fill" hops. Pocket-fill hops are explained in more detail later in this section.

We also varied the mesh sizes $(72 \times 12,72 \times 24$ and $72 \times 36$ in the singly refined lattice) and tracked the movement of a grain boundary over 1000 runs, and averaged the results (Fig. 4.7).

The good fits again confirm that grain boundaries propagate in a random walk fashion. It is interesting to note that the diffusion rate appears to decrease as the width of the periodic cell is increased. Our explanation for this is as follows: First, we represent the grain boundary movement as a series of "steps" each with the same length, $l_{\text {step }}$. In reality, different processes move the grain boundary by different amounts, so this "step" represents an average step over all significant processes. After $N_{\text {steps }}$ steps, we 


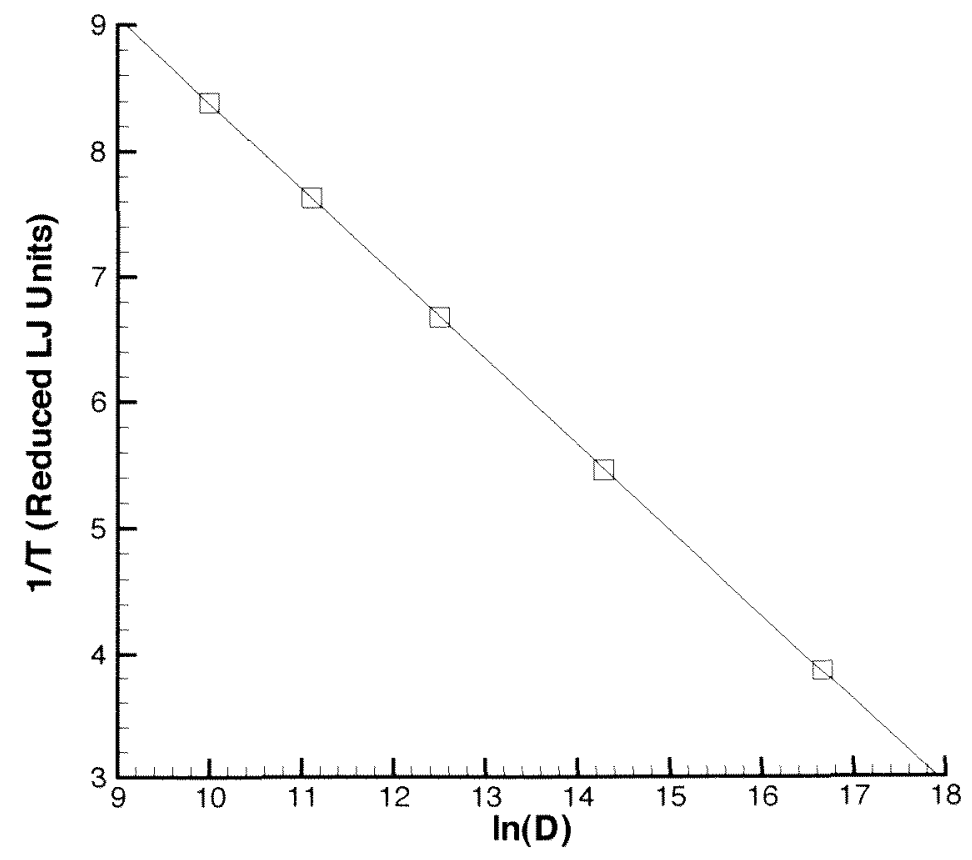

Figure 4.5: Arrhenius plot of grain boundary propagation under the LJ potential. The slope of the fit is $-0.68 \mathrm{E}$ 


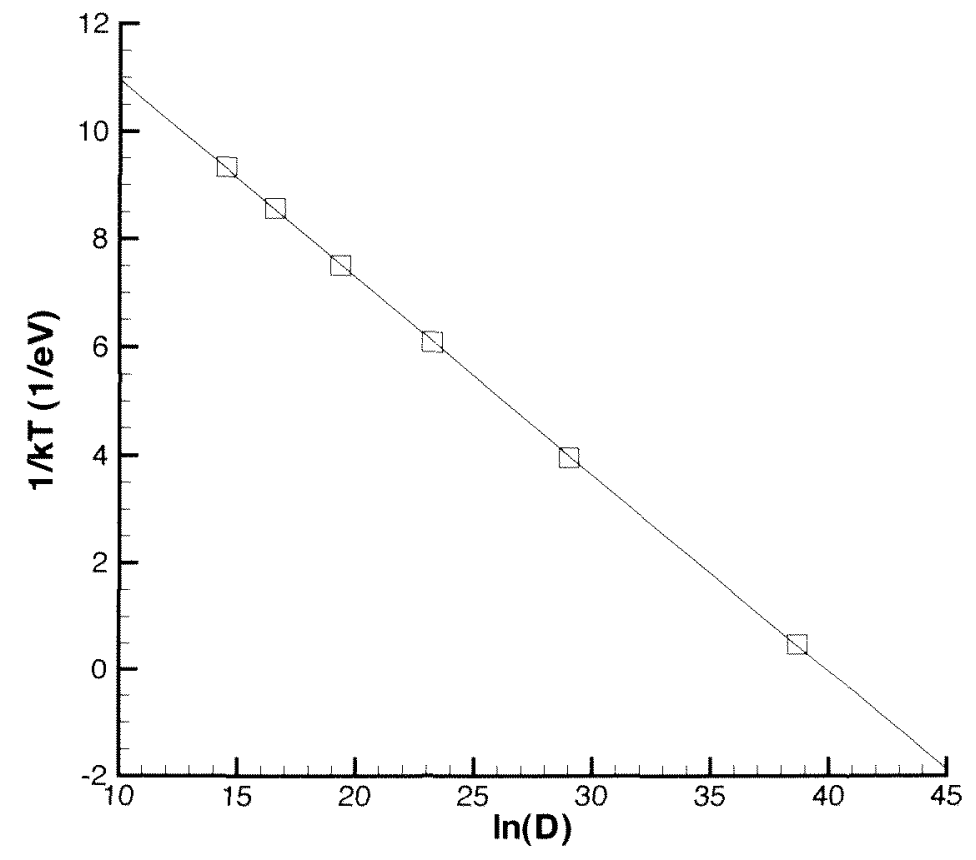

Figure 4.6: Arrhenius plot of grain boundary propagation under the IrSC potential. The slope of the fit is $-0.37 \mathrm{eV}$ 


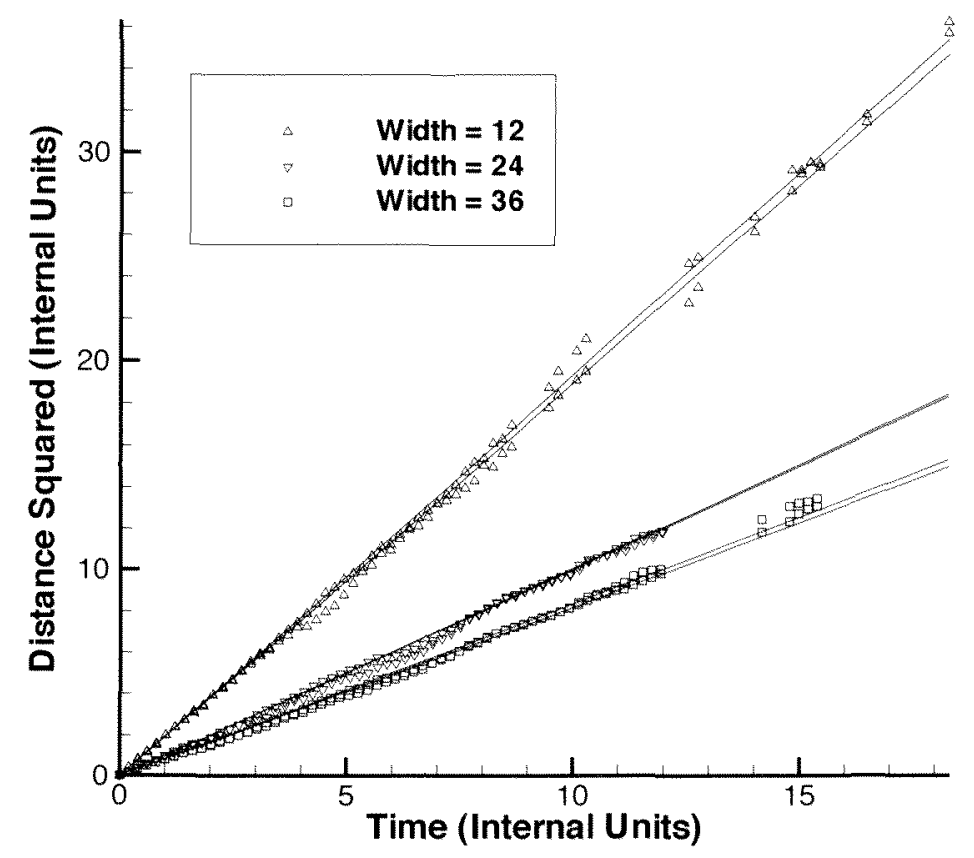

Figure 4.7: Diffusion of the grain boundary under the LJ potential for various lattice widths, averaged over 1000 simulations. The simulations represented by up-triangles have a width of 12 , the down-triangles a width of 24 and the squares a width of 36 . There are two lines for each width, as there are two grain boundaries. 
should expect the mean-squared-distance the grain boundary has moved to be

$$
<x^{2}>=N_{\text {steps }} l_{\text {step }}^{2}
$$

as it is a random walk. We should expect the number of steps performed in some period of time is proportional to the width of grain boundary, i.e.

$$
N_{\text {steps }} \propto w
$$

because if the grain boundary is twice as wide, there should be twice as many opportunities for kink-flip (or other) events. We should expect the contribution of each of these steps to be be similar to

$$
l_{\text {step }} \propto \frac{1}{w}
$$

as each event only moves a portion of the grain boundary, and a grain boundary that is twice as wide will require twice as many events to move the same total distance. Putting these together into Eqn. 4.3 gives

$$
<x^{2}>\propto \frac{1}{w}
$$

which decreases with the width of the periodic cell.

\section{Hops in Grain Boundary Propagation}

To explain the strong dominance of FCC or HCP, we examined the hops that were performed during the propagation (but not merger) of the grain boundaries. We found only two types of hop were performed in this simulation of flat grain boundary propagation, illustrated in Fig. 1.5. These are the kink-flip hop and another hop we refer to as the pocket-fill hop. The kink-flip hops move A-gaps along the boundary. When two A-gaps meet, they set up the possibility for a pocket-fill hop. The exposed atom is very likely to hop across the grain boundary, dissolving the two A-gaps. 
The main hop for differentiating between FCC and HCP is the kink-flip hop. The energy difference between the kink-flips in each direction is very small. This small difference is somewhat amplified by the exponential term in the rate equation, but it is nevertheless surprising that such a small difference can cause such a consistent effect. Although our NEB algorithm method is only consistent to $\sim 10^{-2} \mathrm{eV}$ or $\sim 10^{-2} \sigma$, our KMC algorithm is sensitive to these small differences. So we did not truncate the barriers to smaller level of precision, as we wanted to show that if the barriers were accurately known, our method would show grain boundary movement in the correct direction.

\section{Hops in Grain Boundary Mergers}

Another difference between grain boundary healing for LJ and IrSC was that the LJ grain boundaries tended to merge into an almost continuous channel (with few if any "bridges"), while the IrSC boundaries tended to merge into a series of gaps (or "lakes") with a large number of bridges crossing them (as shown in Fig. 4.8).

The critical event where the grain boundary must "decide" whether to form a channel or a lake is where the grain boundary "pinches", forming a small A-gap triangle as shown in Fig. 4.9, top. This is a similar situation to a pocket-fill move, except that the pinch site does not have 4 neighbours in the plane. If the atom closest to the pinch hops into the pinch (a "pinchplug" hop), then it forms a bridge between the outer terrace. If, instead, the atoms kink-flip onto the outer terrace, the channel remains as a gap.

For IrSC, the pinch-plug hop has a barrier of $0.09 \mathrm{eV}$, with a barrier of $0.43 \mathrm{eV}$ to reverse the hop, if the atom initially has one neighbour. As the forward barrier is much smaller than the reverse barrier, this is almost a one-way process. The reverse barrier is also larger than the barrier for a kink-flip hop, so it is more likely that the atom's initial neighbour will join the outer terrace than for the pinch-plug to reverse. If the atom's initial neighbour kink-flips to the outer terrace, then it is not possible to reverse 

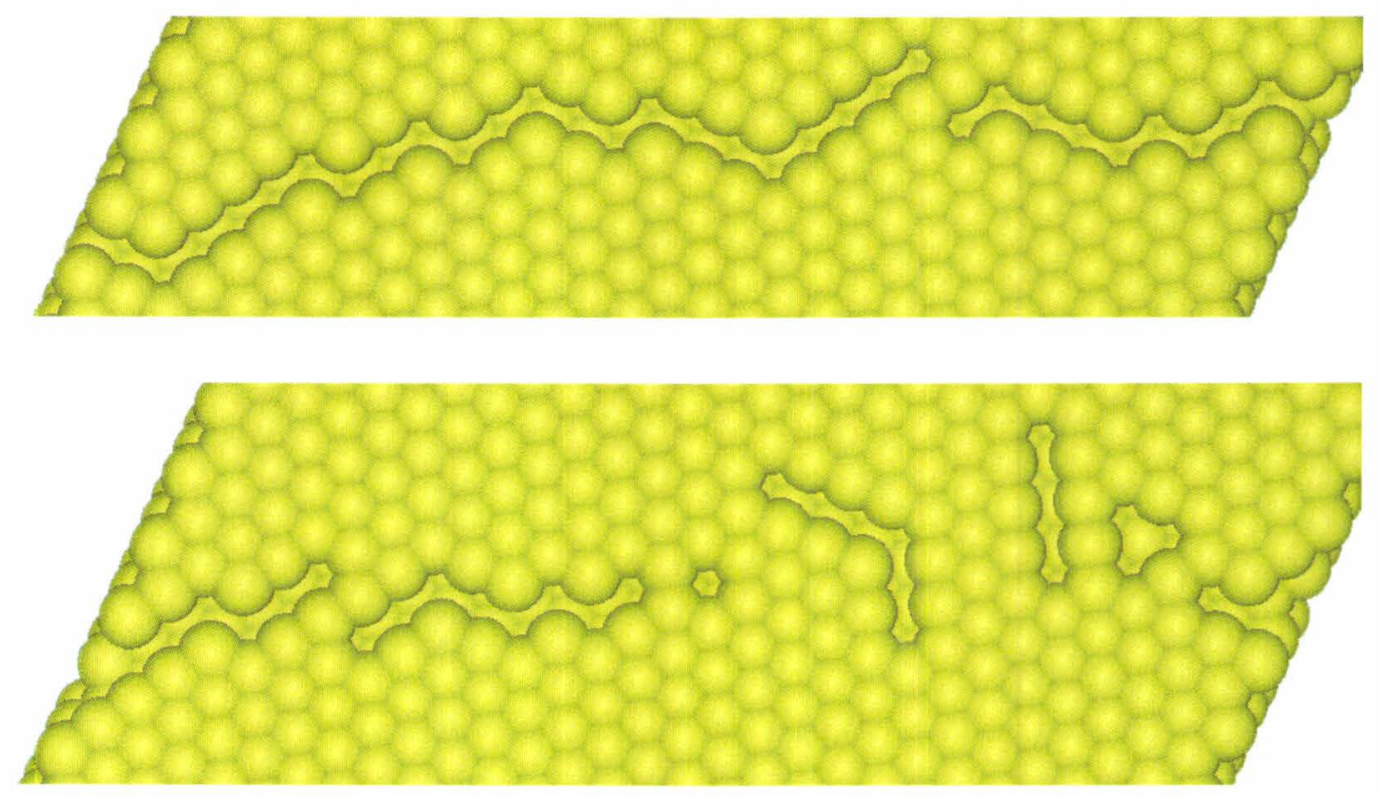

Figure 4.8: Grain boundary shapes after merging. Above: LJ material forms an almost continuous channel after merging. Below: IrSC material forms a series of lakes and bridges. 
the pinch-plug at all, as it would not take the atom to a stable site. This contrasts with the LJ case, where the pinch-plug hop has a barrier of $0.26 \epsilon$ and the reverse pinch-plug hop has a barrier of $0.5 \epsilon$. These values are much closer together, so the hop is more likely to be reversed. Also, the reverse pinch-plug hop has a smaller barrier than the kink-flip, making it more likely that the pinch-hop will reverse before the atom's initial neighbour can kink-flip into the outer terrace, preventing the reverse hop.

If the atom initially has two neighbours (Fig. 4.9, bottom), it is less likely to perform a pinch-plug. However, it is still more likely for an IrSC atom to perform a pinch plug than LJ, (Table. 3.2). Furthermore, the atom closest to the pinch is wedged between two A-gaps, and so can not kinkflip on to the outer terrace until one of its two neighbours has already kink-flipped, putting it in the one neighbour situation described above.

\subsubsection{Enclosed Islands}

Another initial condition we investigated was a small island of FCC or HCP surrounded by material of the opposite stacking type - hence the grain boundary goes around the perimeter the island. We investigated square, triangular and hexagonal islands (Fig. 4.12). of various sizes. Invariably, the enclosed material was assimilated onto the outer material (Fig. 4.13), regardless of which stacking type was enclosed. This demonstrates that the geometry of the grain boundary (e.g. whether the boundary encloses a region) has a stronger effect on the direction of grain boundary propagation than the arrangement of stacking types. The corners always moved first, as shown in the example in Fig. 4.10. Atoms in an internal corner are more likely to move than an outer atom because they have fewer neighbours, and hence a smaller barrier energy to making a hop. Having fewer neighbours is a natural consequence of being part of an enclosed object, as demonstrated in Fig. 4.11.

As the island shrinks, its perimeter size decreases. However, the grain 

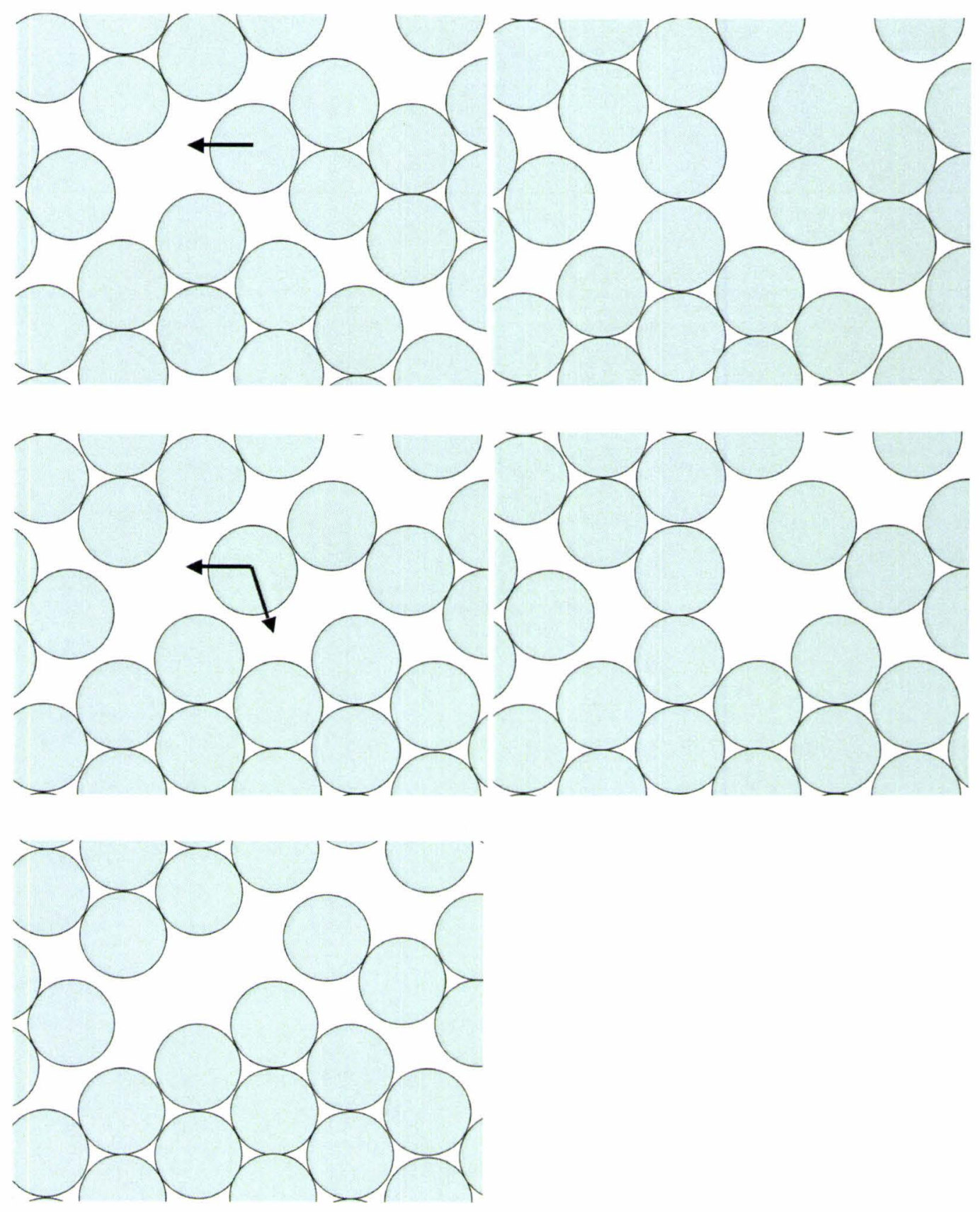

Figure 4.9: Possible mechanisms for the formation of a "bridge" across the grain boundary. Top: The corner atom has only one possible move, going from 2 neighbours on the corner to 3 neighbours in the pinch. It is likely that it will perform this move. Middle: Several atoms behind the corner atom have kink-flipped away, and now it has a choice between kink-flipping (growing the channel, bottom) and pinch-plugging (forming a bridge, middle-right). The atom is more likely to "choose" the pinchplug site under the IrSC potential than under the LJ potential. 
boundary can not shrink with the island, as there is no deposition in this simulation and the amount of free space must be conserved. This is performed by converting A-gaps into B-gaps (which have twice the empty area), and by leaving behind channels. Each of these channels is formed by an A-gap. As atoms in A-gaps are fixed, a long A-gap is only mobile at the ends where there are B-gaps. The atoms at the ends can kink-flip across the grain boundary, but only in a direction away from the centre of the long A-gap (Fig. 4.10). Eventually, only one of the atoms that was on the internal side of the A-gap is left, and it has two possible outer sites to enter. Whichever one it enters, it will leave a one atom wide gap. The atoms in the next layer kink-flip to either side of this small gap. This process continues and a channel is formed.

During deposition, many HCP and FCC islands form, as will be seen in the next section. These islands then grow and meet and interact with each other. If islands of one stacking type grow faster than islands of the other stacking type, they will envelop the other islands. The surrounding material will assimilate the inner islands, even if the barrier energies of hops relevant to grain boundary propagation prefer the opposite stacking type.

\subsubsection{Effect of Coverage}

It was not possible to achieve realistic deposition rates within computationally convenient times, so in our deposition simulations, the rate of deposition was much faster than the rate of grain boundary propagation. When islands meet, the grain boundaries are not able to propagate as they are blocked by the large numbers of atoms that have been deposited on the higher levels of the island. A more realistic scenario is one where the grain boundary is able to propagate a large amount before new atoms are deposited. To simulate the limit of slow deposition, we randomly placed a number of adatoms on the double grain boundary substrate described 


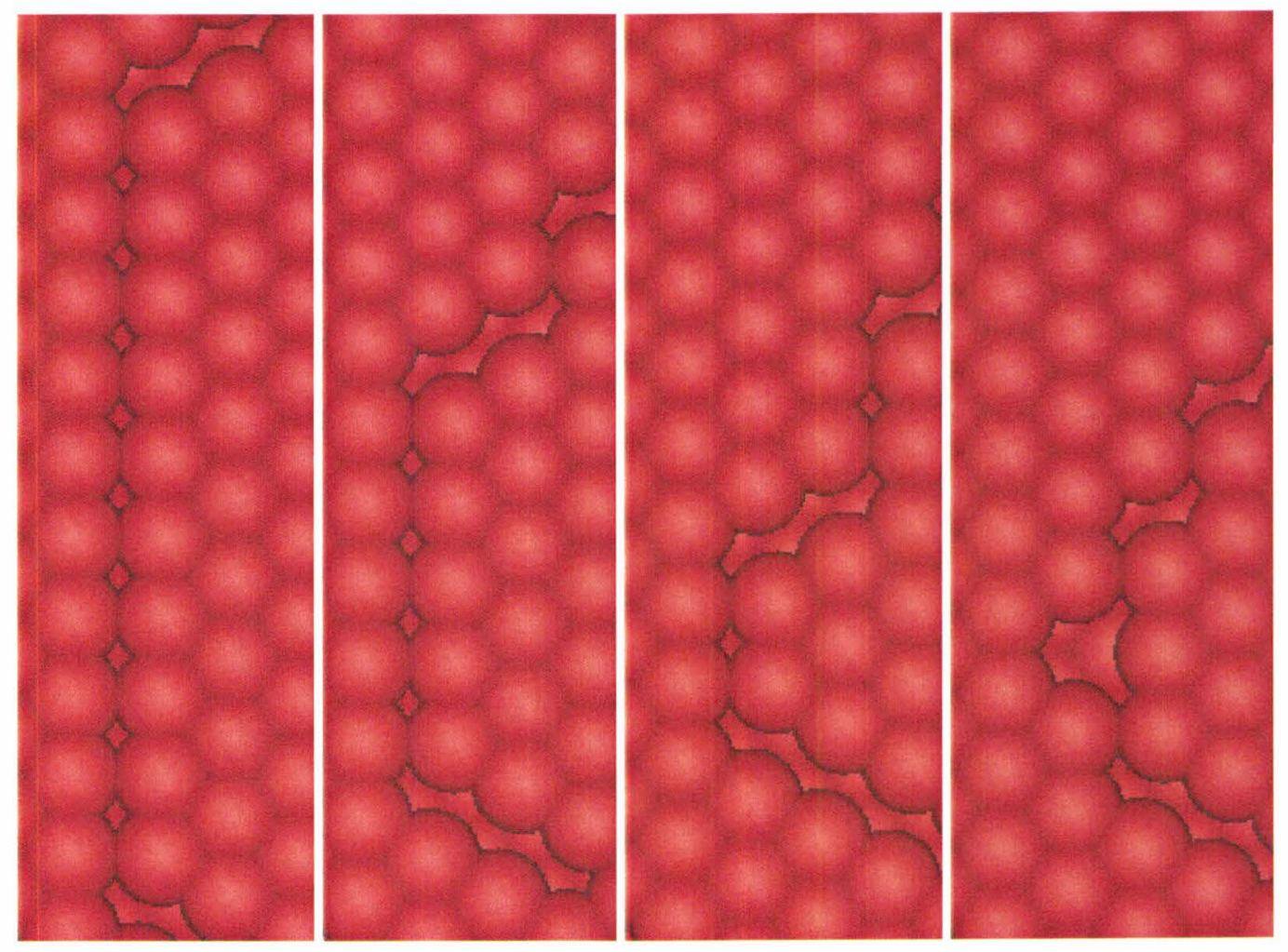

Figure 4.10: An A-gap grain boundary heals, leaving behind a small gap that will become a channel 


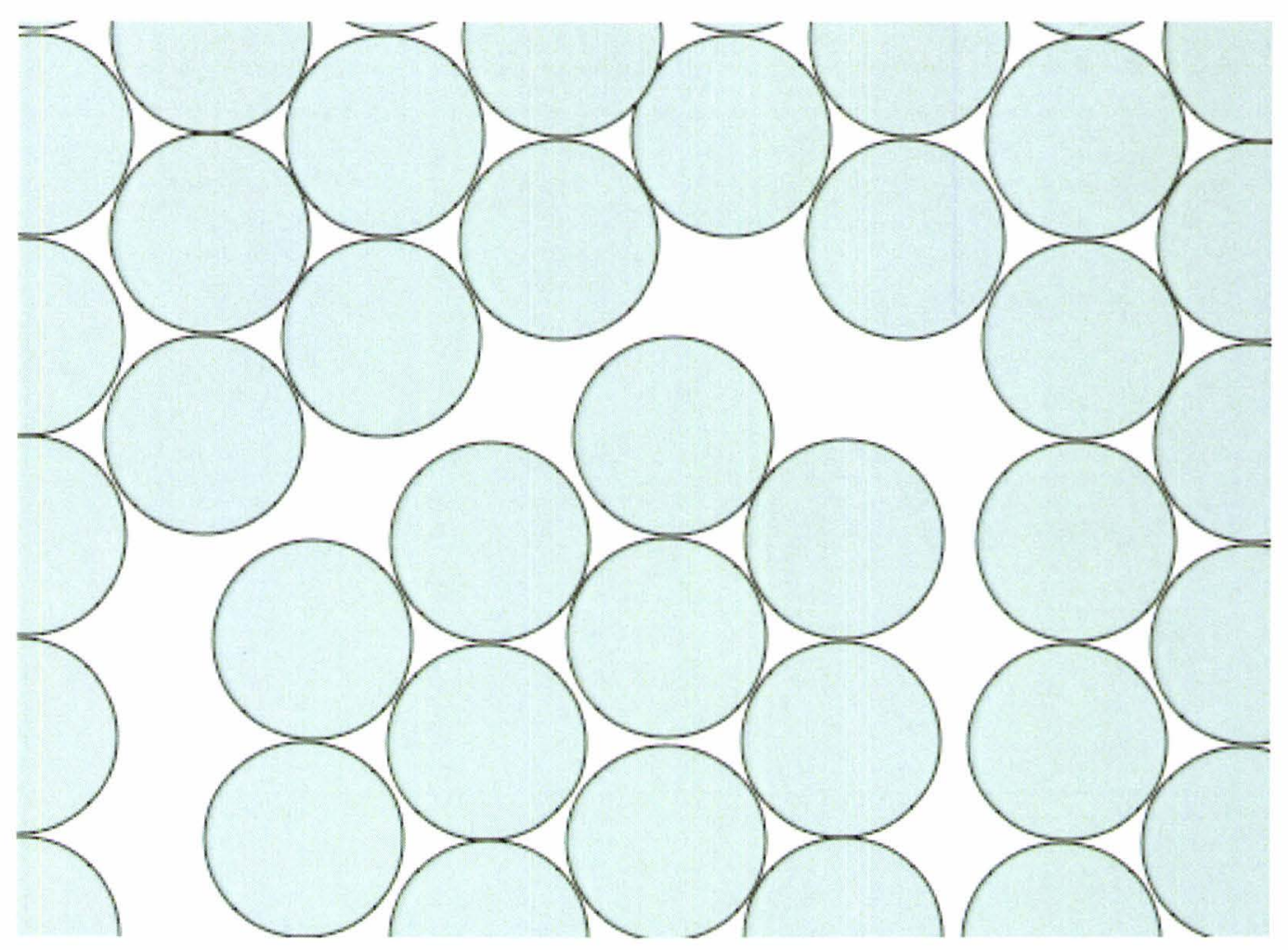

Figure 4.11: Any enclosed shape is taken over by the surrounding stacking type. The atoms in straight edges all have 4 neighbours, but the corner atoms on the inner island only have at most 3 neighbours while the outer corner atoms have 4 or 5 . This makes the inner island less stable. 

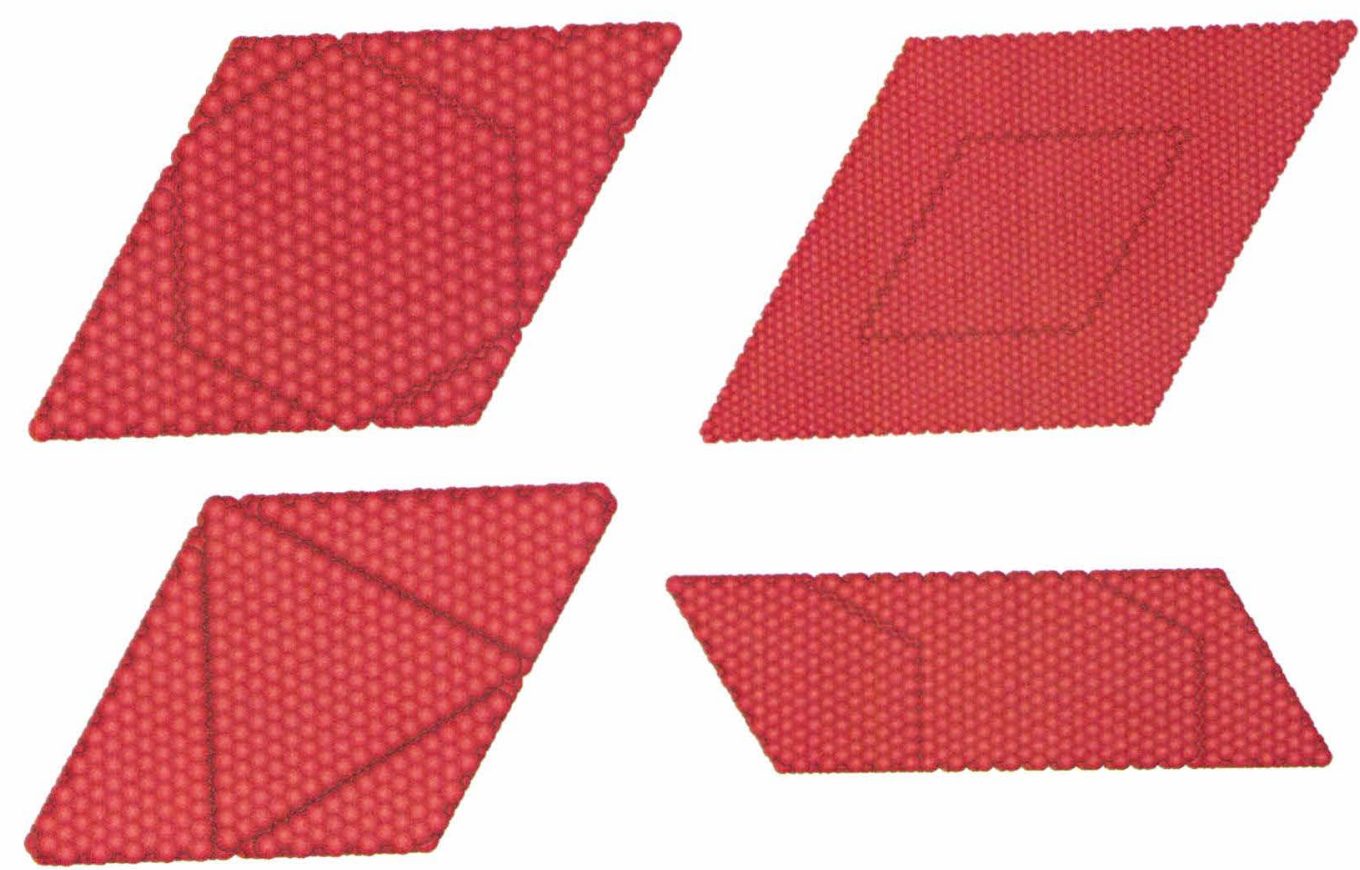

Figure 4.12: Examples of grain boundary shapes investigated. Clockwise from the top left, they are: enclosed hexagon island, enclosed square island, double chevron grain boundary, and enclosed triangular island. 

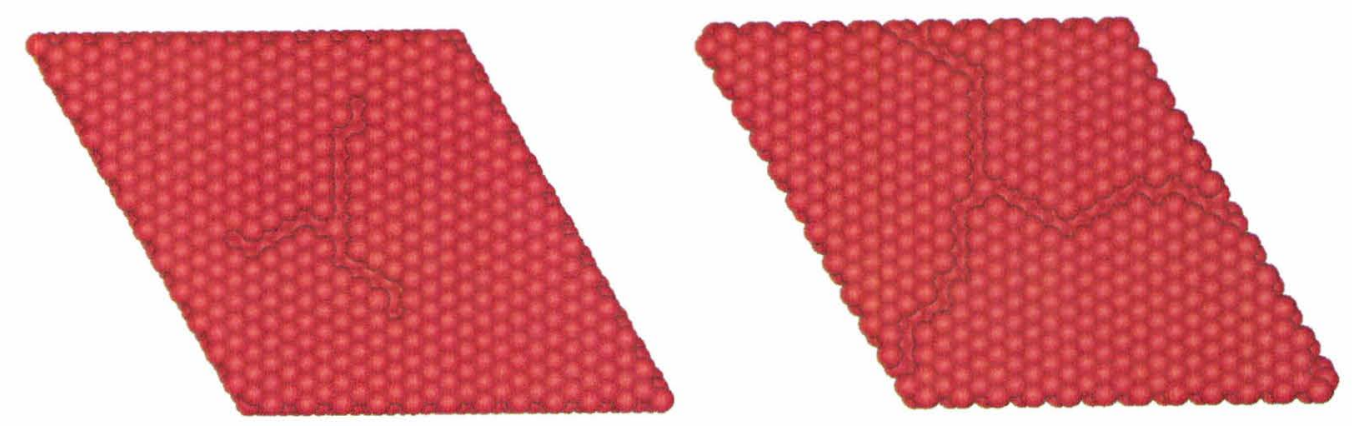

Figure 4.13: A triangular island of HCP (left) and a triangular island of FCC (right) are both taken over by the surrounding stacking type, leaving a triangular channel pattern. Note that the channels for when FCC has enclosed are larger than for the HCP case. This is because in this particular case, the FCC triangle was surrounded by B-gaps while the HCP triangle was surrounded by A-gaps. B-gaps are larger than A-gaps, and the total gap area on the surface is conserved through the grain boundary propagation (as we are ignoring deposition), so an initial configuration with B-gaps gives larger channels than an initial configuration with A-gaps. 

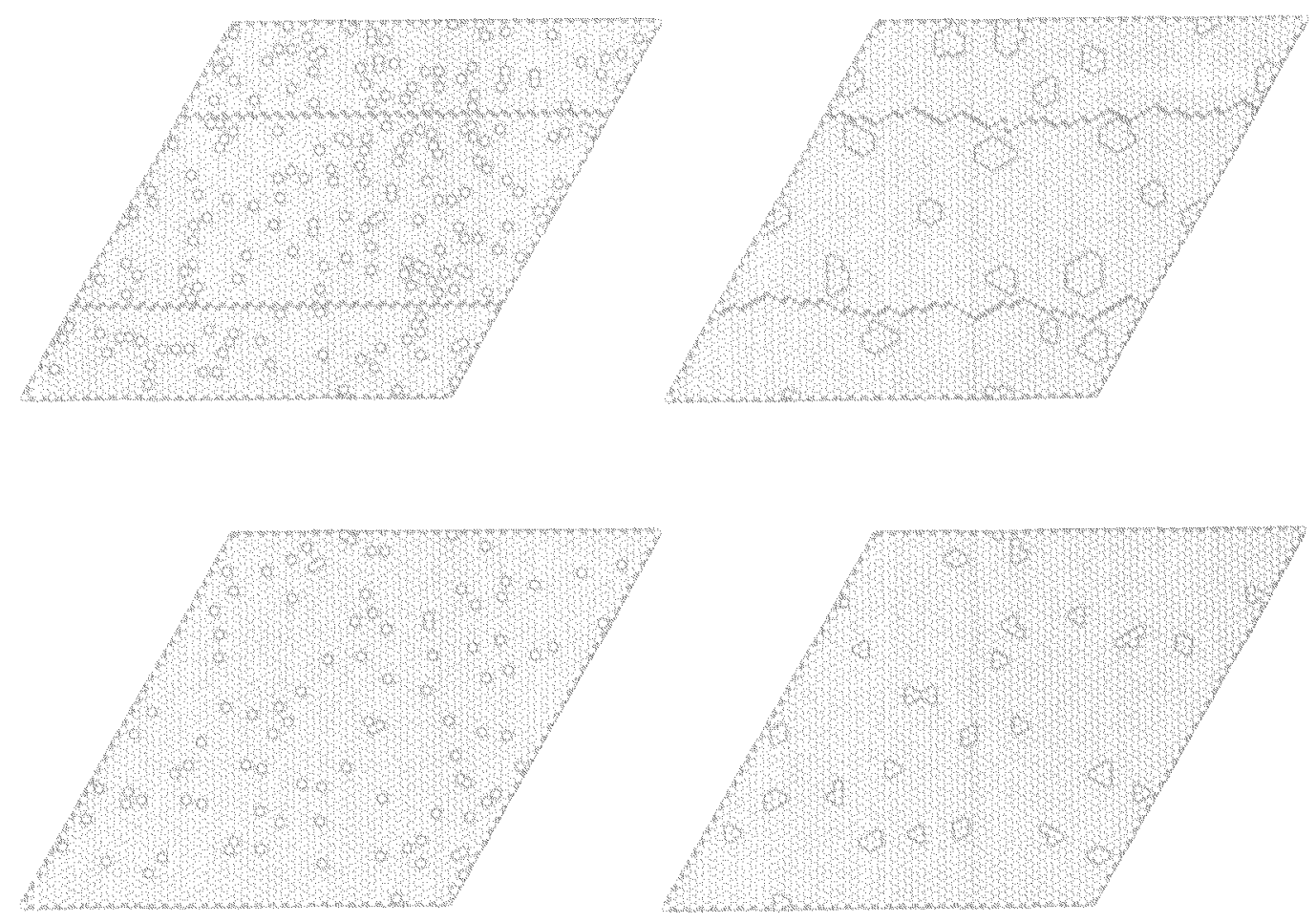

Figure 4.14: Two examples of a random distribution of adatoms quickly forming into islands.

in section 4.1.1, and did not drop any more atoms during the simulation. Simulations were run under the IrSC potential, at $300 \mathrm{~K}$ and $1000 \mathrm{~K}$.

Very quickly the adatoms collected into a number of small islands (Fig. 4.14), of about 3-10 atoms each, with roughly triangular or hexagonal shapes. Because a large number of adatoms were planted instantly on the substrate, this differs to the behaviour observed during deposition where large triangular islands form. When placed one at a time the adatoms diffuse around until they meet an already nucleated island, and rarely will a number of adatoms meet to form a new island. When placed simultaneously, the odds that an adatom will encounter enough other adatoms to nucleate a new small island is much larger, simply because there are more adatoms that have not joined islands yet. 
We noticed that groups of 3 atoms were the smallest that formed, and these triangular trimers were only metastable. Structures on (111) planes of an FCC/HCP substrate have A-edges or B-edges, in the same way that grain boundaries have A-gaps and B-gaps. When two A-edges meet, they form an A-gap and when two B-edges meet they form a B-gap. Trimers surrounded by B-edges (Fig. 4.15) were even able to diffuse over the surface, even though only one atom moves at a time. Trimers surrounded by A-edges were more stable. This is because atoms in a B-edge are capable of moving to an adjacent site while still remaining fairly close to one of its neighbours from the island $-\sqrt{3} \approx 1.7$ times the nearest neighbour distance (Fig. 4.15). Atoms in an A-edge trimer must completely break bonds with both neighbouring atoms in order to move (Fig. 4.15). Once an atom is only semibonded to only one other atom in the island, the barrier for it to completely break away is smaller, and this allows trimers to break up.

Larger islands also break up, particularly at the higher temperature (i.e. $1000 \mathrm{~K}$ ). Typically, the atoms that have left a smaller island will join another island, making it larger. This is similar to the process of Ostwald ripening [52]. Systems typically act to reduce their surface area and hence their surface energy. In KMC, surface energy can be represented by the barrier energies for the outer atoms of an island to step away from the island. Atoms in a straight edge have more neighbours than atoms in the corner of an island, and hence find it harder to leave the island. In fact, under the IrSC potential, atoms with 3 or more neighbours on the plane can not escape the island at all (Fig. 4.16). Smaller islands are less stable than larger islands as the ratio of corner atoms to edge atoms is larger. The act of reducing the surface area reduces the probability that an atom will leave the structure, increasing its stability.

Many other metastable structures such as strings (Fig. 4.17) formed. These quickly reform to reduce their surface area in a similar manner to that mentioned above. 


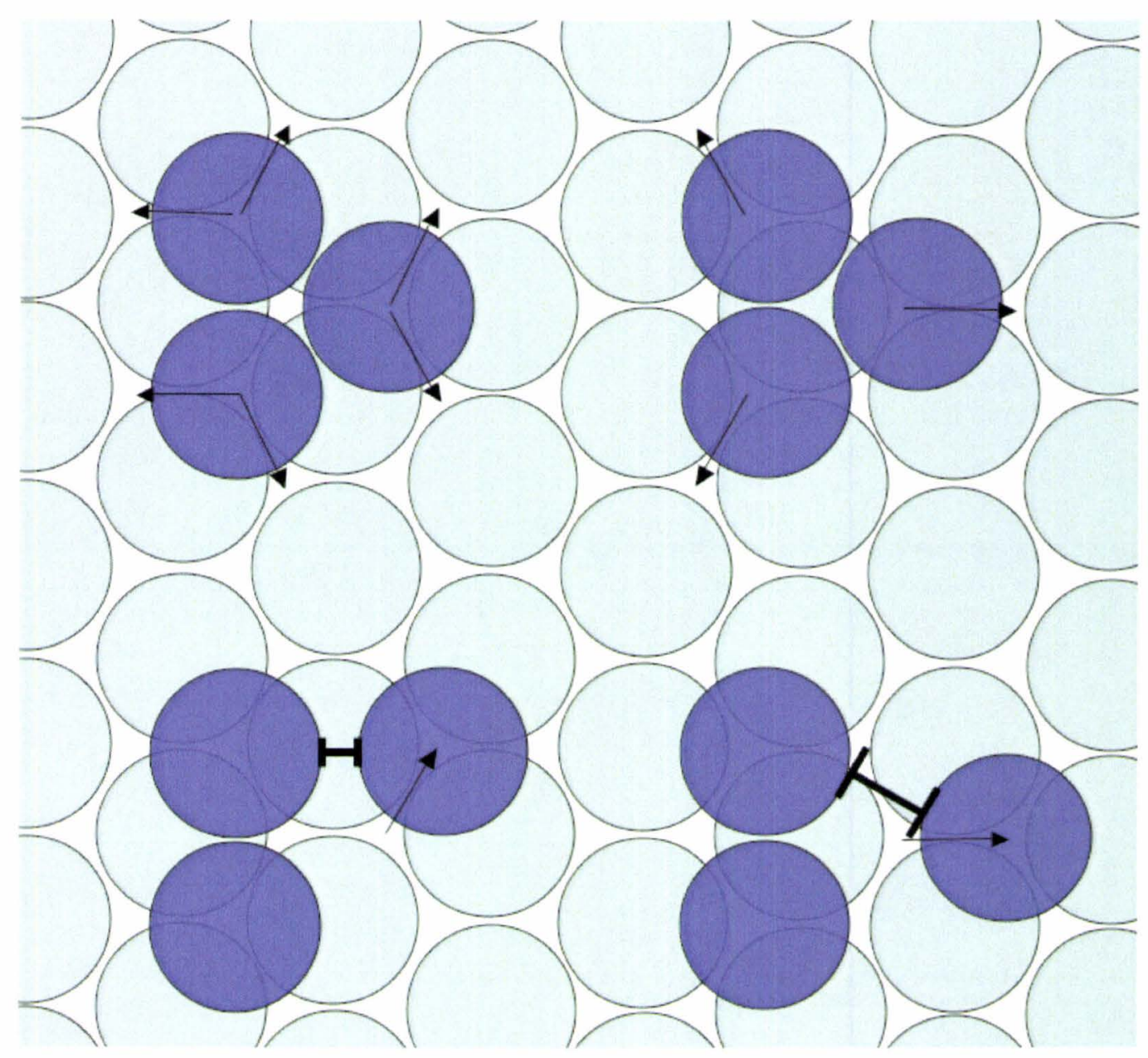

Figure 4.15: Top left: a B-gap trimer, showing all possible moves. Top right: an A-gap trimer, showing all possible moves. Bottom left: A B-gap trimer after a move. Note the small distance between the atom that moved and its closest atom. Bottom right: An A-gap trimer after a move. Note the larger distance between the atom that moved and its closest atoms. 


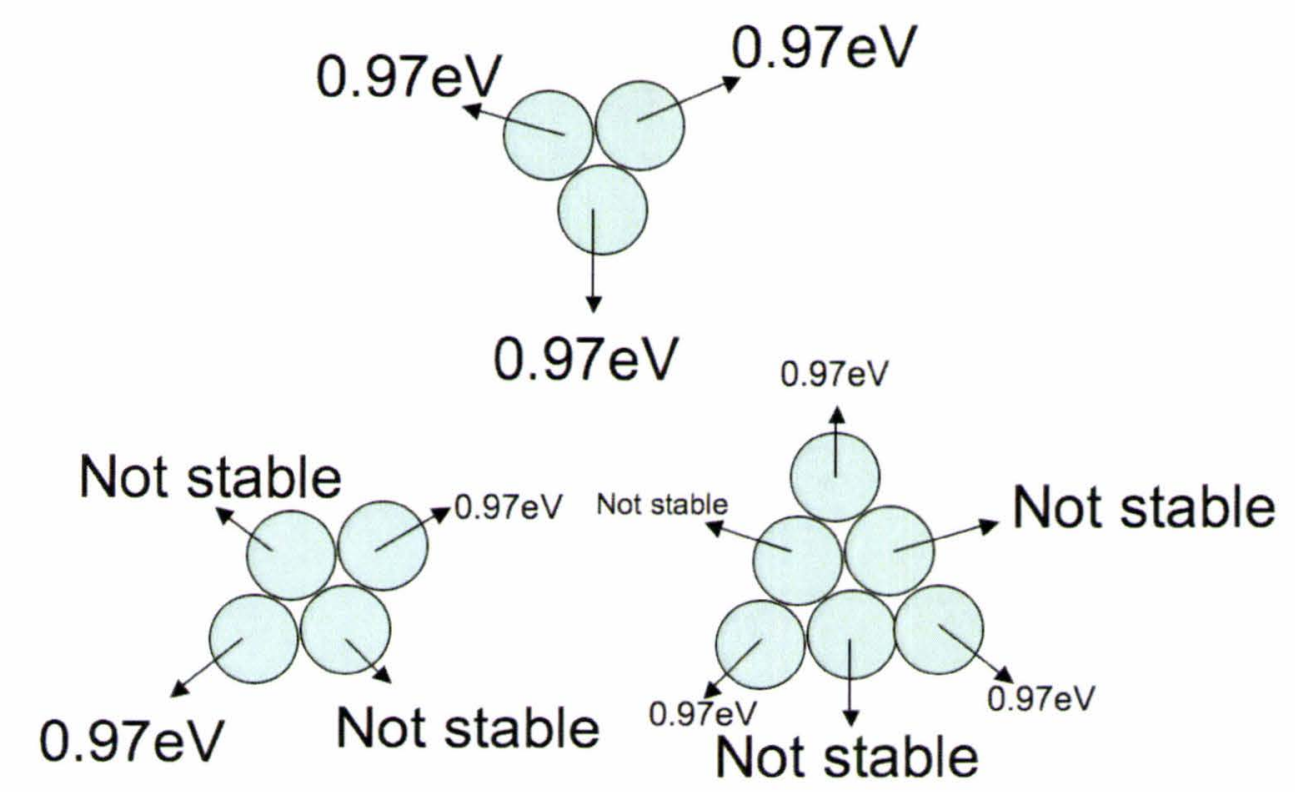

Figure 4.16: An illustration of the instability of small islands. Corner atoms have an escape energy of $0.97 \mathrm{eV}$, but atoms with 3 or 4 neighbours can not escape, as the closest site is unstable and they would immediately fall back into the island. 


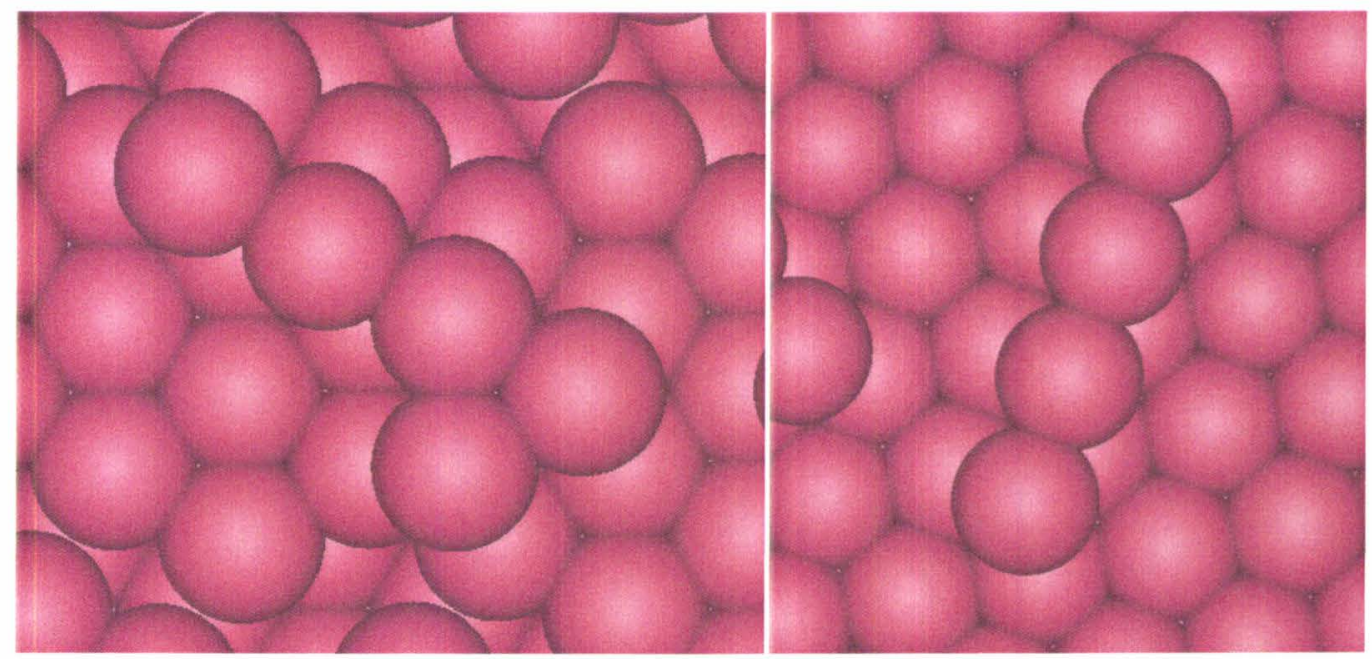

Figure 4.17: "String" structures form from a random distribution of adatoms within $\sim 2 n s$ and last for $\sim 10 n s$. This behaviour was observed under both the LJ and IrSC potentials.

\section{Decorated Sites}

We also observed atoms moving into decorated sites. Decorated sites are surprisingly stable $-0.43 \mathrm{eV}$ to hop in versus $0.94 \mathrm{eV}$ to hop out, if there are no neighbours. This means that atoms sometimes hop out of the edge of an island on to a decorated site. We observed a pentamer island dissociating into decorated atoms in this way (Fig. 4.18).

Adatoms or island-edge atoms that have hopped into a decorated site tend to remain in them. These decorated atoms can slide (barrier of 0.97 $\mathrm{eV}$ if there are no neighbours) along the top of the A-gap, but not onto B-gaps. This means that the decorated atoms are localised within some region of the grain boundary. As we have not permitted atoms that are supporting other atoms to move, this means that the decorated atoms pin the grain boundary, preventing it from moving.

Decorated atoms are more likely to hop back onto HCP or FCC sites if there are many neighbours in the FCC/HCP site. This would suggest that the side of the grain boundary with a greater adatom coverage will 
receive more adatoms from decorated sites than the other side would. We have not explored the significance of this for grain boundary propagation and self-healing.

\subsection{Deposition}

We set up large (mesh sizes up to 576 by 576) flat slabs 2 layers high and performed deposition simulations. We noticed no significant difference between the two potentials. We found that the deposited adatoms form triangular dendritic islands (Fig. 4.19), similar to those shown in STM images of island growth on (111) Iridium (Fig. 4.20). FCC and HCP islands face in opposite directions, also in agreement with experiment. However, the detailed shapes of the islands beyond the general triangular outline is quite different. It is possible that this is because our NEB calculations were generally performed in conditions most similar to an atom on a grain boundary - using this to simulate island growth processes is an extension beyond these conditions. Furthermore, we are not taking into account concerted movements of atoms, such as the diffusion of dimers, which may have an effect on island growth.

Due to limited simulation time, it was necessary to use a very high deposition rate $\left(\sim 10^{11}\right.$ monolayers $/ \mathrm{s}, \sim 10^{13}$ times that of experiment [10]). This meant that many adatoms deposited on top of a growing island do not have time to make it over the Ehrlich-Schwoebel barrier and attach themselves to the edge of the island before they encounter enough other adatoms to form their own small island upon the larger island. This process continues, and the islands turn into terrace structures (Fig. 4.21). The higher levels prevent grain boundary propagation when the islands meet, stopping the self-healing process.

To confirm that the Ehrlich-Schwoebel barrier was causing this effect, we performed a deposition simulation with the Ehrlich-Schwoebel barrier "switched off" - we set the barrier energy for an atom to step off an island 

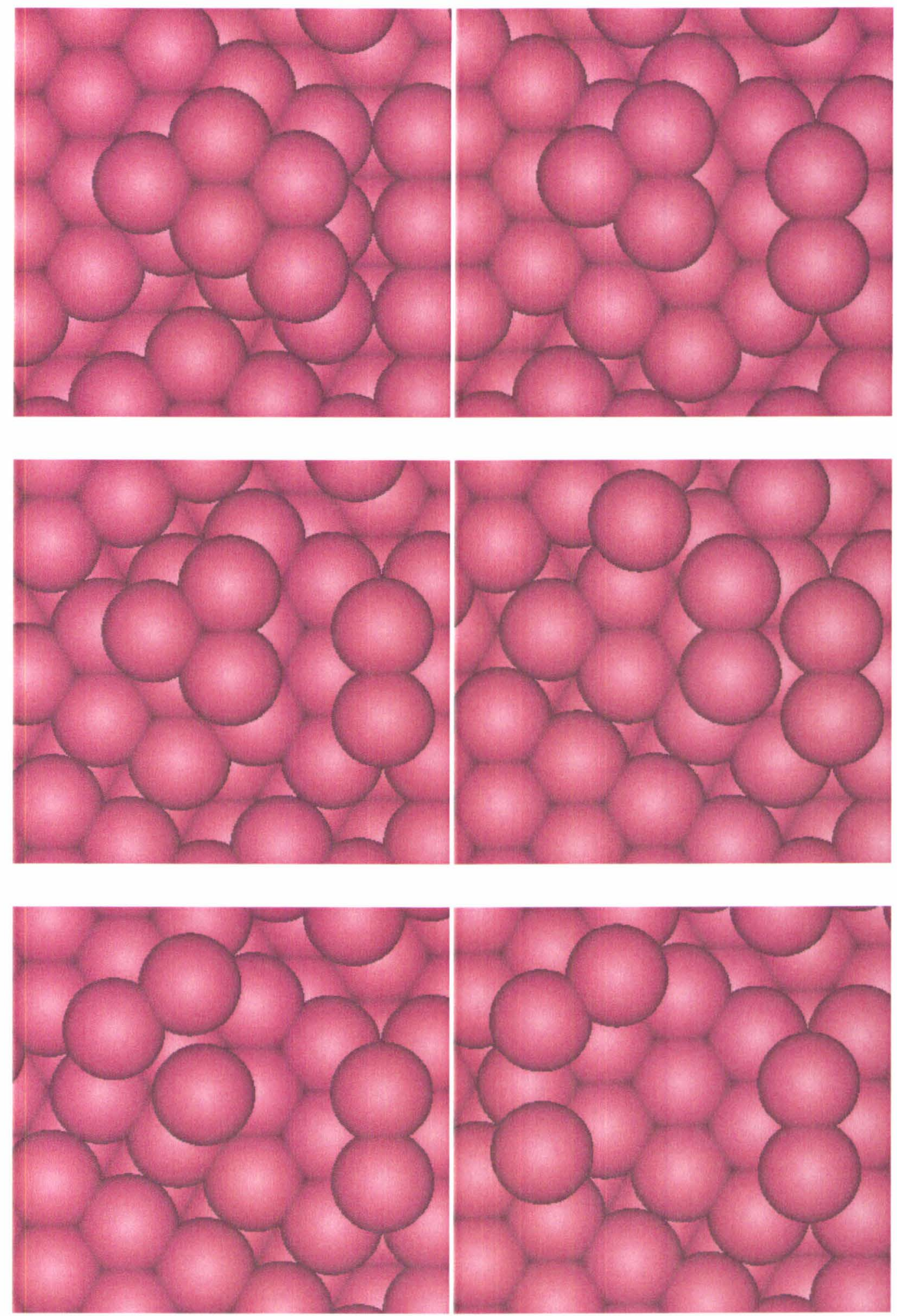

Figure 4.18: A sequence of images demonstrating the dissociation of a pentamer island into decorated atoms. The whole process occurred over $\sim 50 \mathrm{~ns}$. 


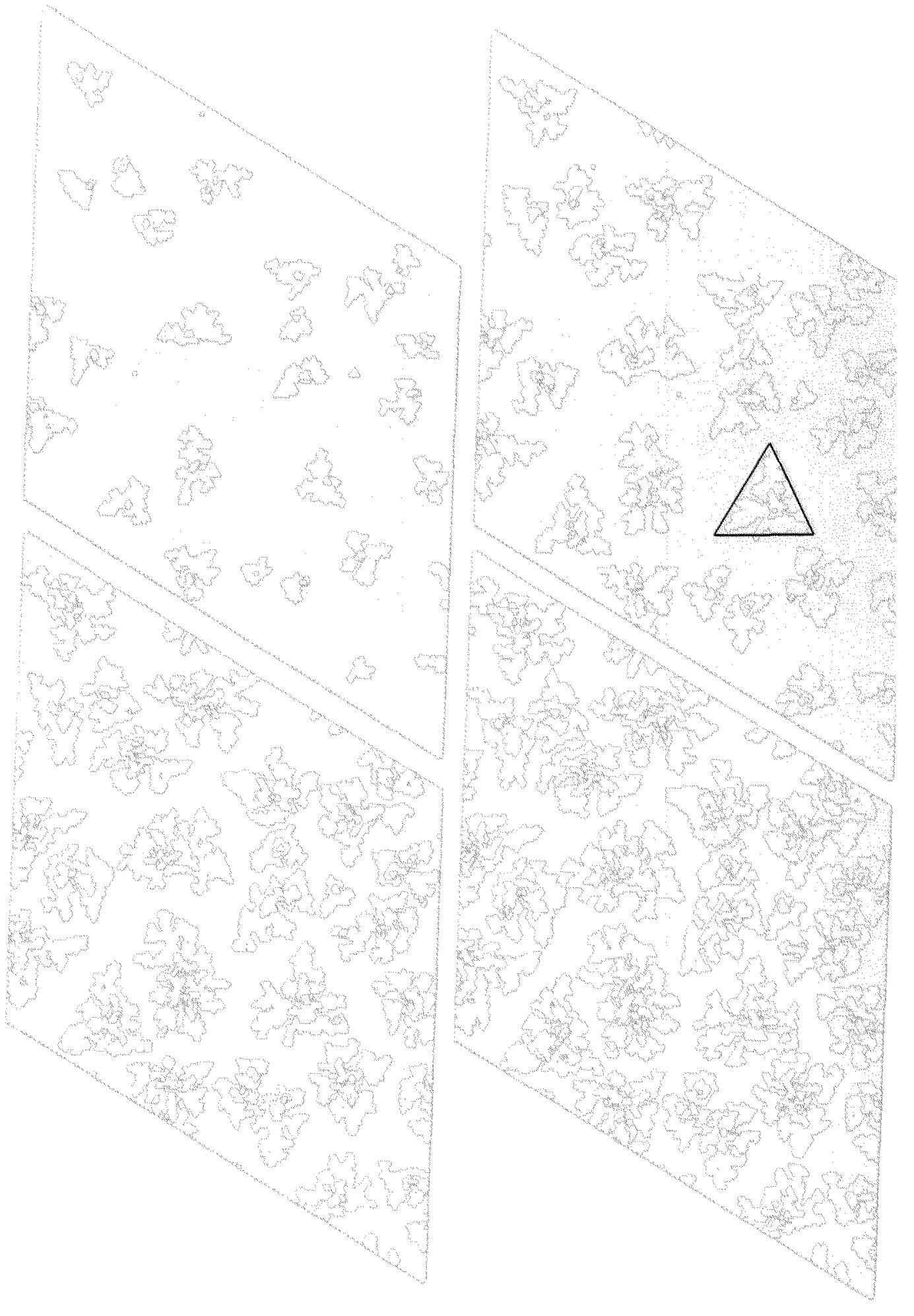

Figure 4.19: An example of deposition upon the (111) plane of a periodic slab. Islands form roughly triangular shapes, with FCC islands rotating $180^{\circ}$ clockwise from HCP islands. The dominance of the EhrlichSchwoebel barrier causes terraces to form. Although these terraces form in experiment, our high deposition rate increases their abundance. 


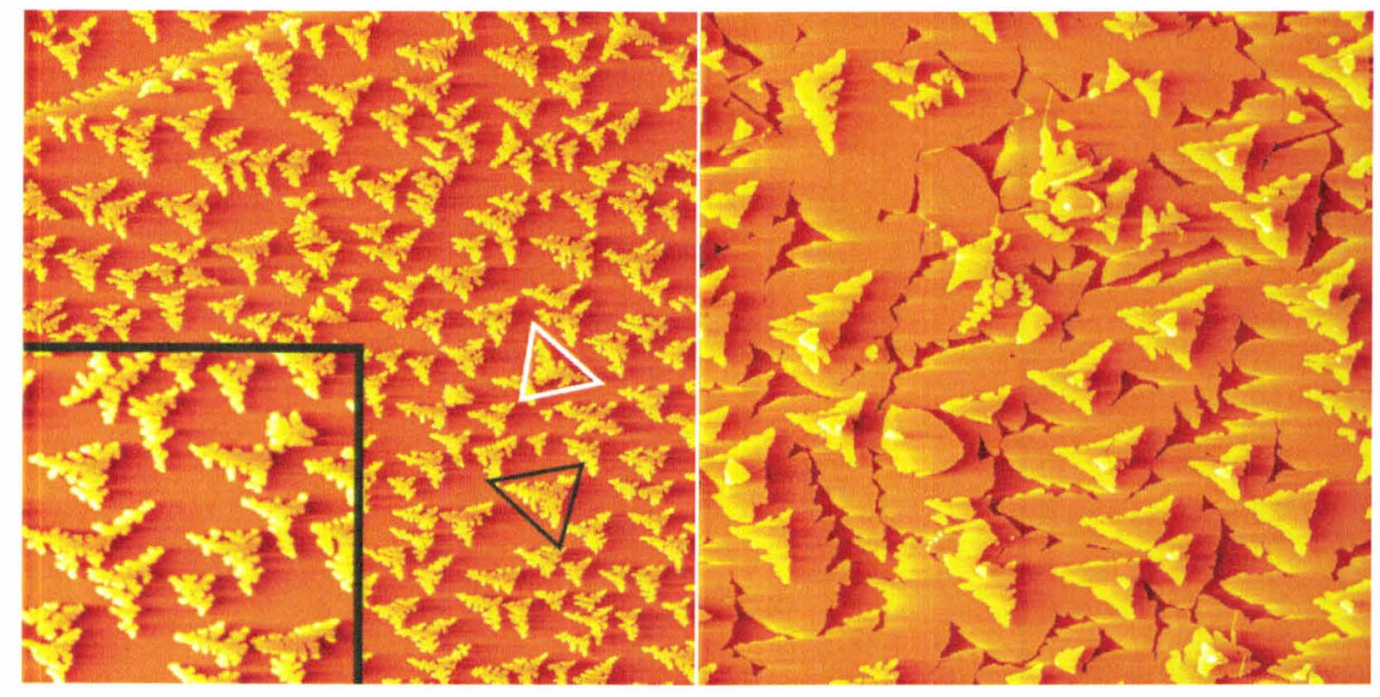

Figure 4.20: STM images of deposition on (111) Iridium [11]. Left: An early stage of deposition at $350 \mathrm{~K}$. HCP islands are rotated $180^{\circ}$ with respect to FCC islands. The triangular envelope for an HCP island is shown in white, while the envelope for an FCC island is shown in black. Our simulation size is roughly the size of the inset. Right: A later stage of deposition at $450 \mathrm{~K}$. The islands are still triangular, but are larger. Also, some atoms are forming terraces on higher levels. 


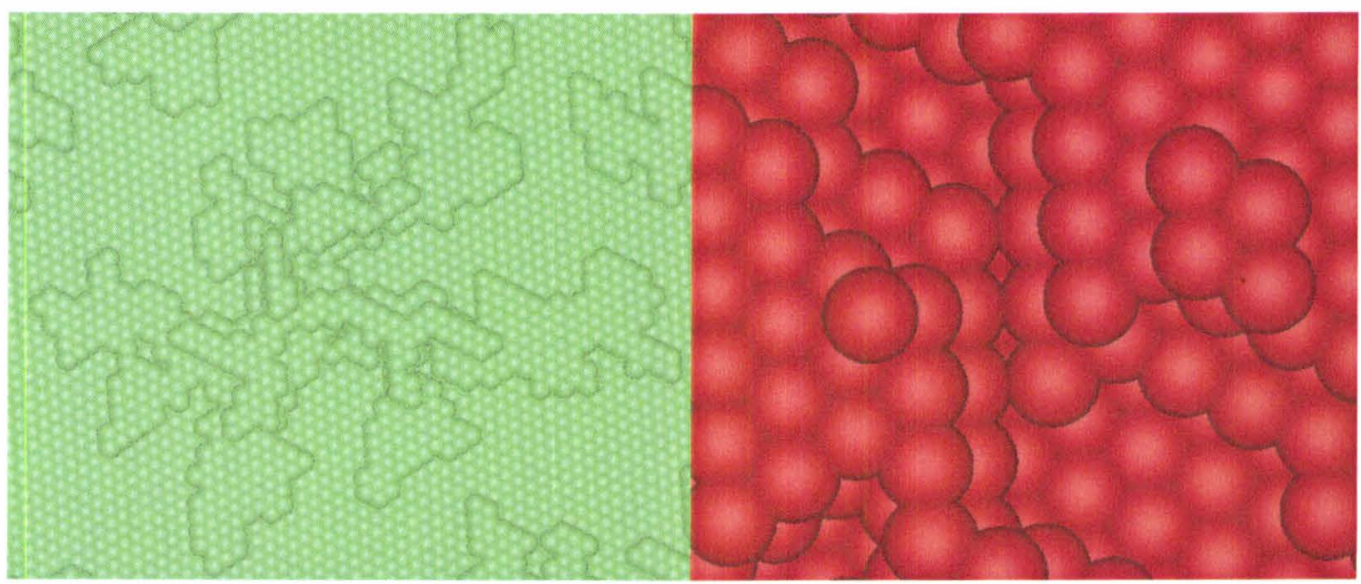

Figure 4.21: Left: An island containing triangular elements, with several layers of island on top. Right: When two terraced islands meet, they get stuck

equal to the barrier energy for an adatom to diffuse to an adjacent site on a flat surface. In this artificial and unrealistic scenario, no islands were nucleated on top of other islands. Instead, the atoms attached themselves to the edges of already nucleated islands, causing flat island growth. When regions of HCP met regions of FCC, grain boundaries formed. These propagated until the entire neat FCC layer was formed. This "cheat" shows similar behaviour to what has been experimentally observed to occur at lower deposition rates, so we can conclude that the Ehrlich-Schwoebel barrier is what prevents self-healing at high deposition rates. 


\section{Chapter 5}

\section{Conclusions}

We found that KMC is a good method for simulating the processes of deposition and grain boundary propagation. If the energy barriers are accurate, it can demonstrate the effects of a preference for stacking fault or regular sites. Our NEB algorithm is a good method for finding barrier energies, but we found it so sensitive to the configuration of atoms a long distance from the hop that we must conclude it is only accurate to about $\sim 0.01 \epsilon$ or $\sim 0.01 \mathrm{eV}$. This is larger than the difference between FCC and HCP hops, so a different method will be necessary to find these details. Second-neighbours may have make a significant difference on the scale of the difference between FCC and HCP sites, so the look-up table may need to be extended to examine these hops in detail.

The barrier energies calculated with the IrSC potential appear to be less sharply affected by changes in the number of initial and final neighbours of a hop than the barrier energies calculated with the LJ potential. We should expect real metals to act more like the IrSC potential. The qualitative differences we observed include a switch between a preference of $\mathrm{HCP}$ and a preference of FCC - and although the difference was smaller than the accuracy of our NEB algorithm (mentioned above), this does demonstrate some difference between the potentials. The most notable difference was a difference in the shape of the gaps formed when grain 
boundaries merge - LJ preferred long channels, IrSC preferred "lakes" and "bridges". However, the differences were not large, and we can conclude that the LJ potential performs reasonably in modelling grain boundary propagation in Ir.

We observed the propagation of grain boundaries and self-healing with a preference for a particular stacking type, as shown in experiment. Deposited islands had a similar triangular shape to that shown by STM images of deposition, but the high deposition rate we used caused the islands to form terraces that prevented the self-healing process.

Further work could include improving the efficiency of the KMC algorithm, to allow longer simulation times, larger lattices and lower deposition rates. This would give us a more realistic picture of the processes occurring in deposition. Adding additional processes - such as diffusion of dimers and trimers - could also increase agreement with experiment. A more consistent system for determining energy barriers is also necessary to correctly model the differences between FCC and HCP sites. A more accurate potential function than IrSC could also be implemented in the NEB code to more accurately model Iridium, or different potentials could be used to investigate different materials without major changes to the algorithm, provided the material has an FCC or HCP structure in bulk. A major extension to this project would be to perform a full simulation of epitaxy using all these improvements, including deposition at a realistic rate, island growth, and grain boundary propagation as islands intersect and layers self-heal, so that the epitaxial growth of FCC Iridium can be better understood. 


\section{Appendix A}

\section{Critical hops}

For reference, all the important hops we mentioned in the text are illustrated in this appendix. 

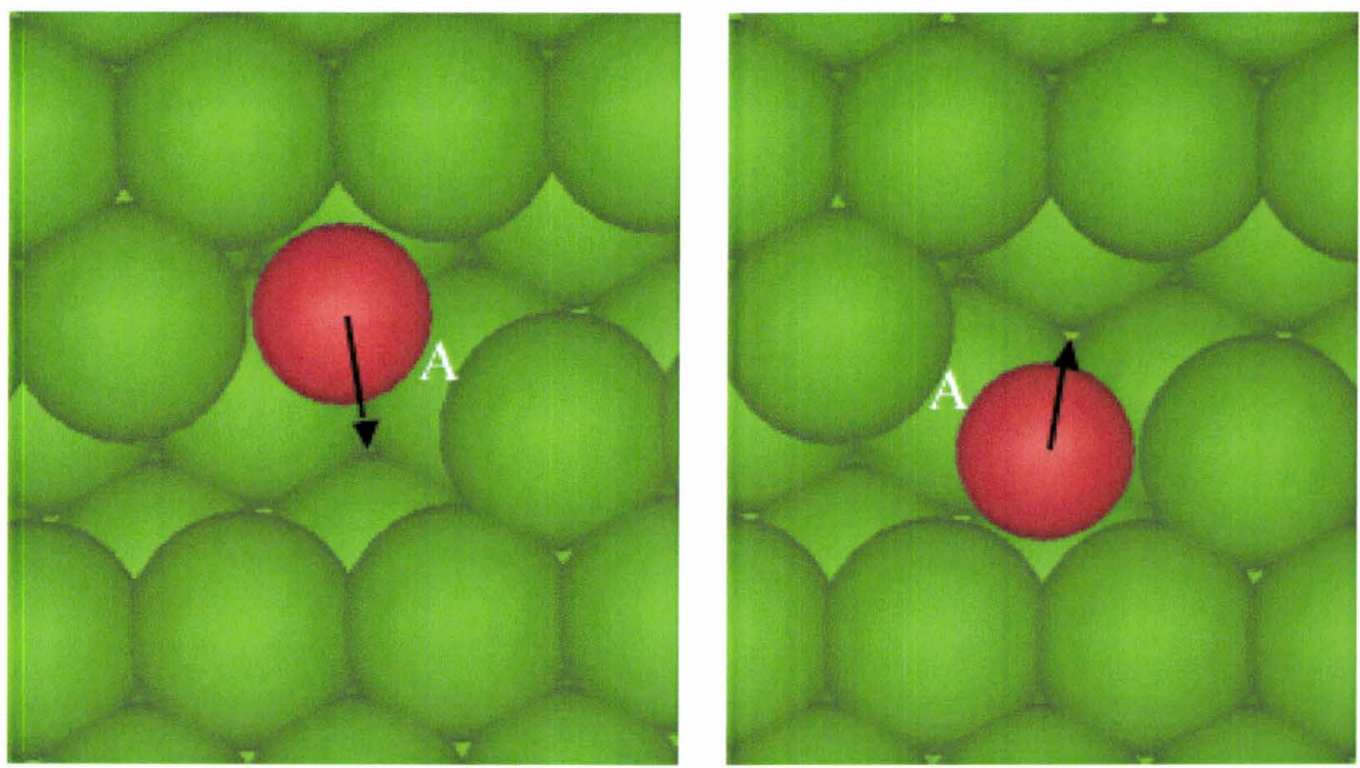

Figure A.1: A kink-flip hop
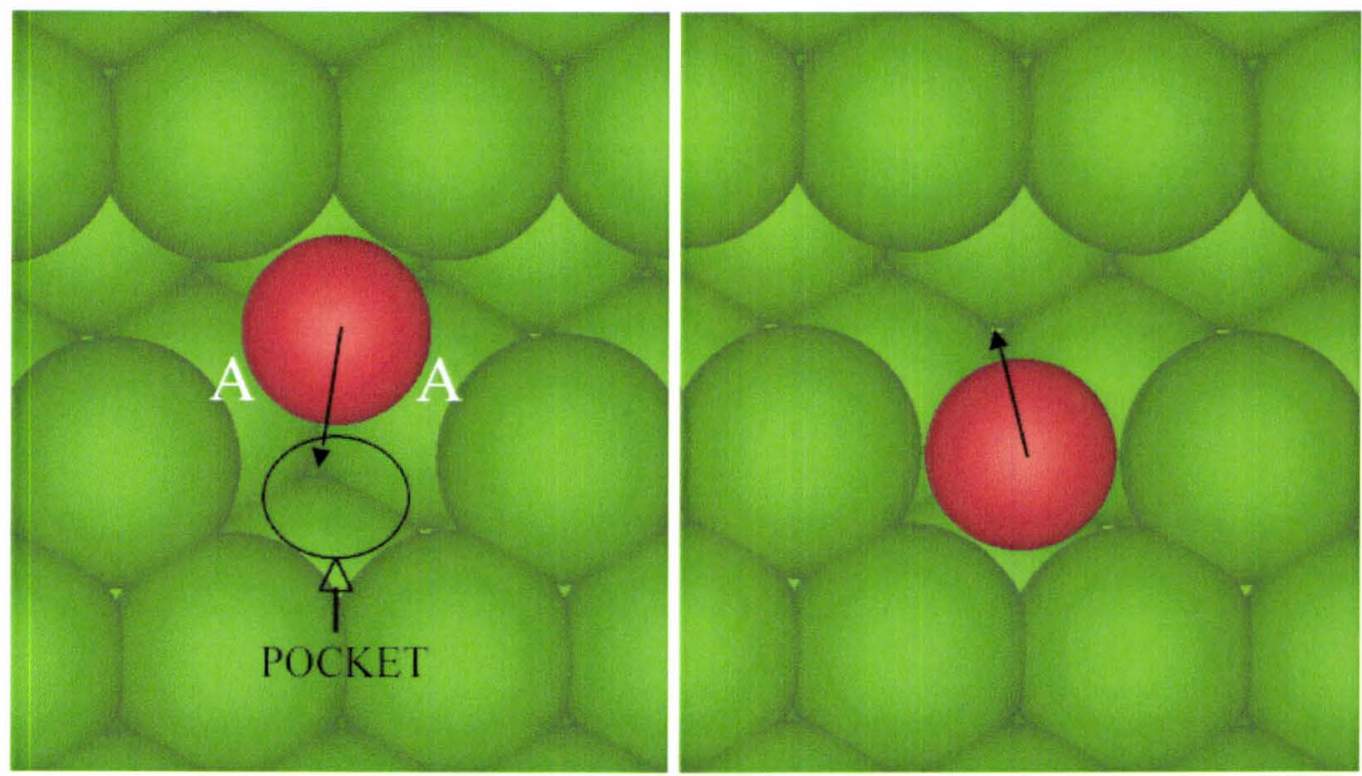

Figure A.2: A pocket-fill hop 

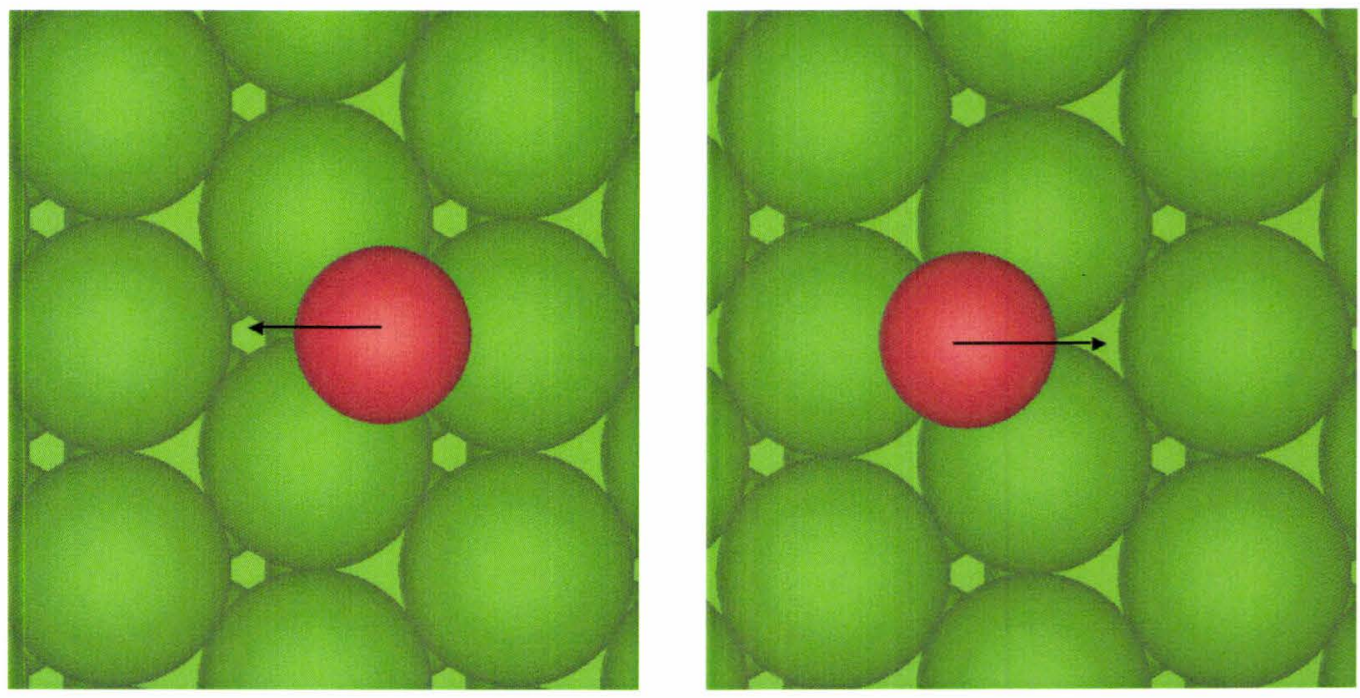

Figure A.3: A diffusion step
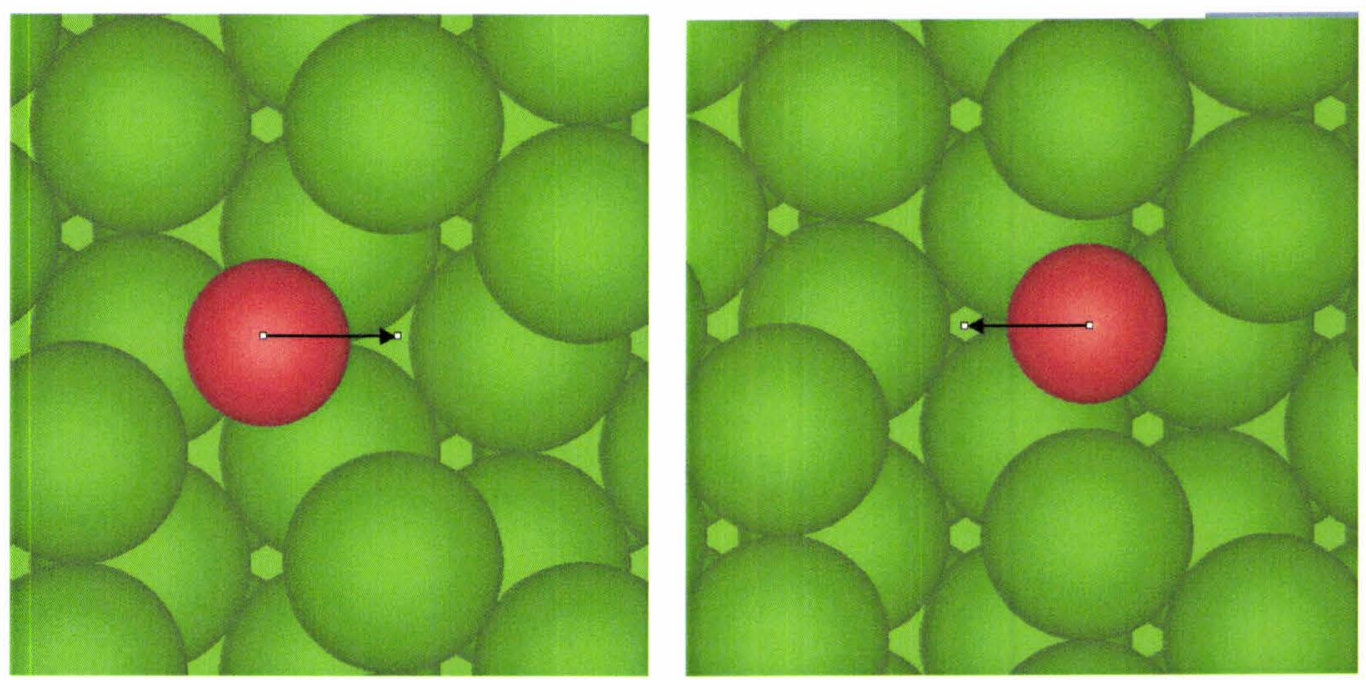

Figure A.4: A pinch-plug, starting with 2 neighbours 

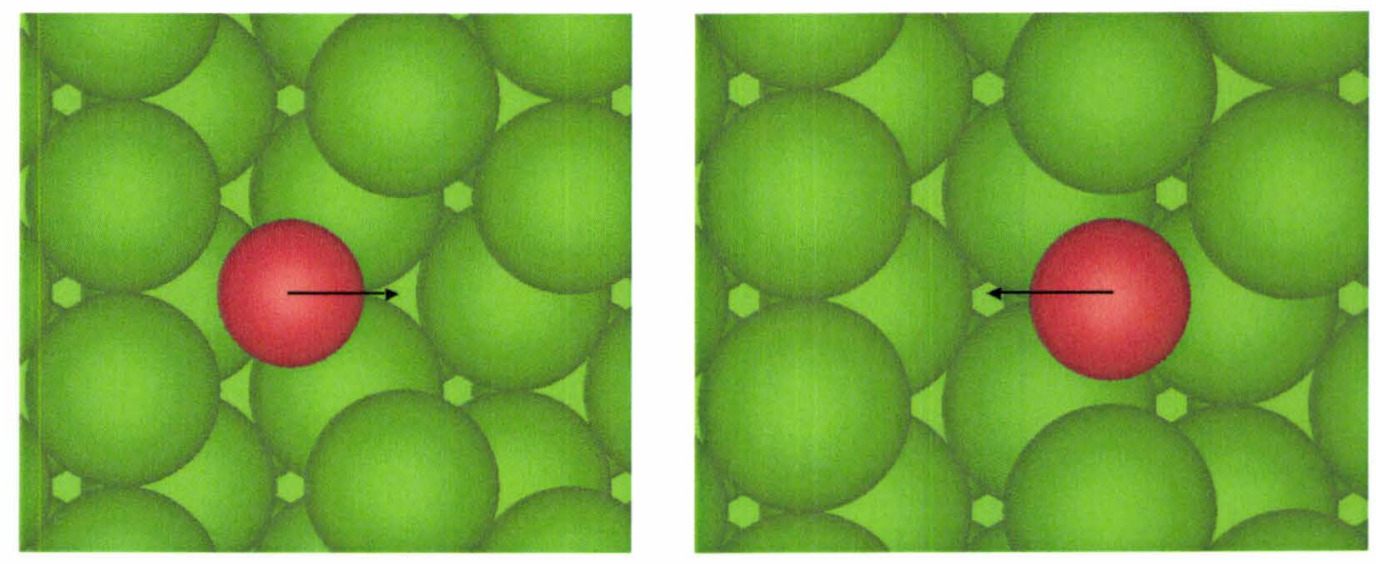

Figure A.5: A pinch-plug, starting with 3 neighbours 


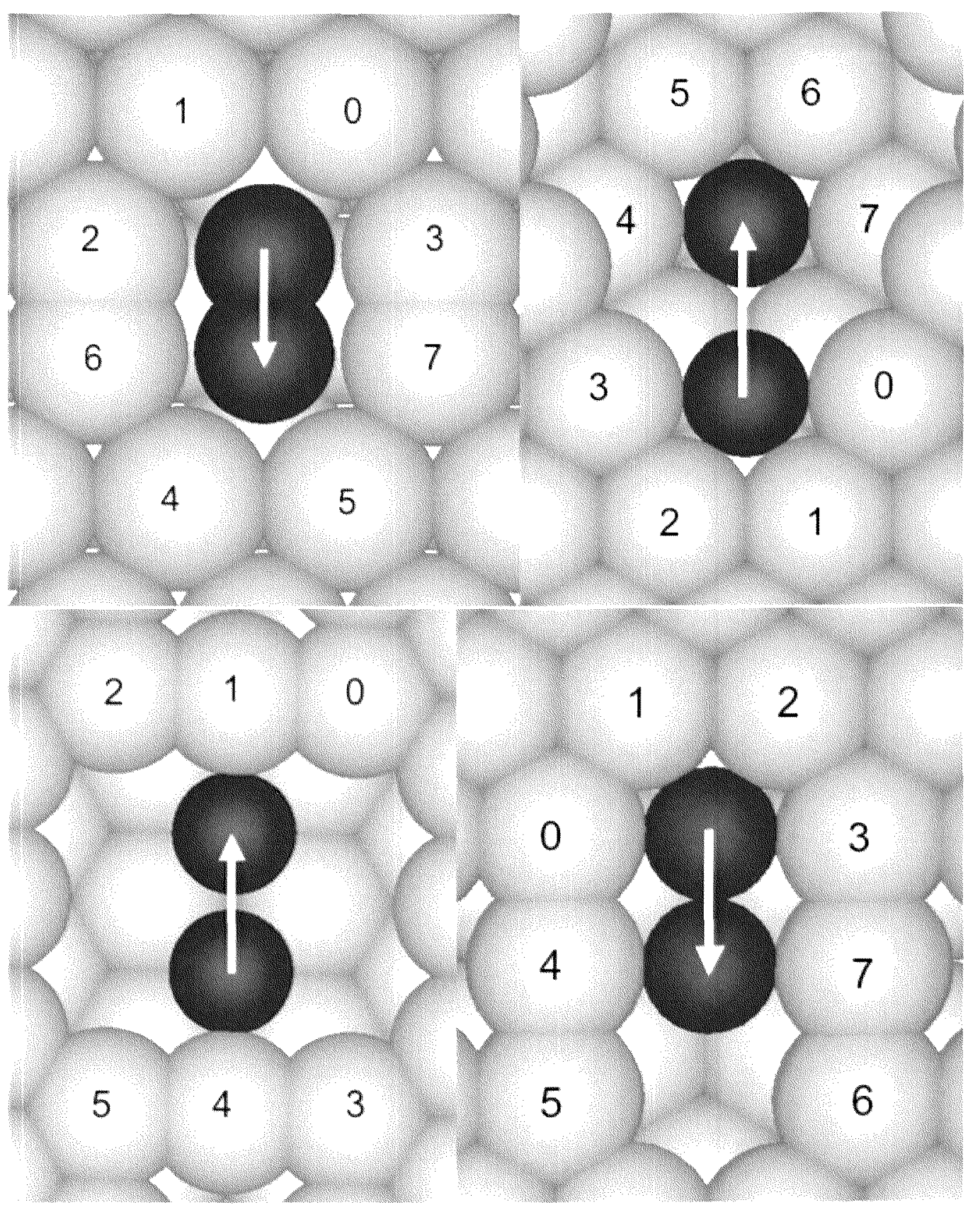

Figure A.6: The ID numbers for the neighbours in the four classes of hop. Except for the drop hop, all of these hops are reversible, and the reverse energy barriers are calculated at the same time as the forward energy barriers. Note that some of the neighbours can not be "switched on" at the same time, because they overlap. Top left: An FCC to HCP short hop. Top Right: A drop off a ledge. Bottom Left: A hop from a decorated site into an adjacent decorated site on the same A boundary. Bottom Right: A hop into a decorated site. The A boundary runs from left to right through the middle of this image. 


\section{Bibliography}

[1] G. May, and S. Sze, Fundamentals of Semiconductor Fabrication (2004).

[2] R. Keyes, Science, New Series 196, 945-949 (1977).

[3] J. Kwo, C. Hsieh, R.M. Fleming, M. Hong, S.H. Liou, B.A. Davidson and L.C. Feldman, The American Physical Society 36, 4039 (1987).

[4] T. D. Khoa, S. Horii and S. Horita, Thin Solid Films 419, 88-94 (2002).

[5] M. Panish, Science, New Series 208, 916-922 (1980).

[6] A. Robinson, Science, New Series 188, 720-722 (1975).

[7] F. Rinaldi, Basics of Molecular Beam Epitaxy (MBE) Annual Report 2002, Optoelectronics Department, University of Ulm

[8] D. Chrisey, and G. Hubler, Pulsed Laser Deposition of Thin Films (1994).

[9] K. Fichthorn, and M. Scheffler, Physical Review Letters 84, 5371 (2000).

[10] C. Busse, C. Polop, M. Müller, K. Albe, U. Linke and T. Michely, Physical Review Letters 91, 056103 (2003).

[11] A. Thoma and T. Michely, Private Communication 91, (2007). 
[12] M. Müller, K. Albe, C. Busse, A. Thoma and T. Michely, Physical Review B 71, 075407 (2005).

[13] R. L. Schwoebel, and E. J. Shipsey, Journal of Applied Physics 37, 10 (1966).

[14] G. Ehrlich, and F. G. Hudda, Journal of Chemical Physics 44, 3 (1966).

[15] C. Busse and T. Michely, Surface Science 552, 281 (2004).

[16] S. Bleikamp, A. Thoma, C. Polop, G. Pirug, U. Linke and T. Michely, Physical Review Letters 96, 115503 (2006).

[17] http://www.ndt-ed.org (Retrieved August 2007).

[18] W.K. Burton, N. Cabrera and F.C. Frank, Philosophical Transactions of the Royal Society of London. Series A, Mathematical and Physical Sciences 243, 299-358 (1951).

[19] V. Fuenzalida and I. Eisele, J. Cryst. Growth 74, 597 (1986).

[20] S. Harris and P. Smilauer, Physical Review B 50, 7952 (1994).

[21] M.P. Allen, and D.J. Tildesley, Computer Simulation of Liquids (2006).

[22] J. Dai, J. Kanter, S. Kapur, W. Seider and T Sinno, Physical Review B 72, 134102 (2005).

[23] F. Much, M. Ahr, M. Biehl and W. Kinzel, Computer Physics Communications $147,226-229$ (2002).

[24] G. Henkelman and H. Jónsson, Journal of Chemical Physics 115, 9657 (2001).

[25] P. Zoontjens, T. P.. Schulze and S. C. Hendy, Physical Review B 76, 245418 (2007). 
[26] E. Bitzek, P. Koskinen, F. Gähler, M. Moseler and P. Gumbsch, Physical Review Letters 97, 170201 (2006).

[27] F. Ercolessi, A Molecular Dynamics Primer Unpublished manuscript, Trieste (1997).

[28] H. Jónsson, G. Mills and K. Jacobsen, 'Nudged Elastic Band Method for Finding Minimum Energy Paths of Transitions', in 'Classical and Quantum Dynamics in Condensed Phase Simulations', ed. B. J. Berne, G. Ciccotti and D. F. Coker World Scientific 385 (1998).

[29] A. Leach, Molecular Modelling: Principles and Applications (2001).

[30] G. Henkelman and H. Jónsson, Journal of Chemical Physics 113, 9978 (2000).

[31] G. Henkelman, B. Uberuaga and H. Jónsson, Journal of Chemical Physics 113, 9901 (2000).

[32] K. Laldler and M. Christine King, Journal of Physical Chemistry 87, 2657-2664 (1983).

[33] N. Combe, P. Jensen and A. Pimpinelli, Physical Review Letters 85, 110 (2000).

[34] K. E. Khor and S. Das Sarma, Physical Review B 62, 16657 (2000).

[35] C. C. Battaile, J. E. Srolovitz and J. E. Butler, Journal of Applied Physics 82, 6293 (1997).

[36] W. H. Press, S. A. Teukolsky, W. T. Vetterling and B. P. Flannery, Numerical Recipes in Fortran 77 (1997).

[37] B. Sadigh, T. J. Lenosky, S. K. Theiss, M. Caturla, T. Diaz de la Rubia and M. A. Foad, Physical Review Letters 83, 4341 (1999). 
[38] F. M. Bulnes V. D. Pereyra and J. L. Riccardo, Physical Review E 58, 86 (1997).

[39] D. Paschek and R. Krishna, Langmuir 17, 247 (2001).

[40] C. Kittel, and H. Kroemer, Thermal Physics (1980).

[41] T. Schulze, Physical Review E 65, 036704 (2002).

[42] N. Ashcroft, and N. Mermin, Solid State Physics (1976).

[43] A. P. Sutton and J. Chen, Philosophical Magazine Letters 61, 139-146 (1990).

[44] J. Cai, and Y. Y. Ye, Physical Review B 54, 8398 (1995).

[45] M. Daw, and M. I. Baskes, Physical Review B 29, 6443 (1984).

[46] P. Hohenberg, and W. Kohn, Physical Review 136, B 864 (1964).

[47] P. Agrawal, B. Rice and D. Thompson, Surface Science 515, 21-35 (2002).

[48] P. Zoontjens, Private Communication (2007).

[49] S.C. Wang, and Gert Ehrlich, Physical Review Letters 62, 2297 (1989).

[50] J. R. Laeter, J. K. Bóhlke, P. Bièvre, H. Hidaka, H. S. Peiser, K. J. R. Rosman and P. D. P. Taylor, Pure Applied Chemistry 75, 683-800 (2003).

[51] C. Busse, Nucleation and stacking-faults on the Iridium (111) surface PhD Dissertation, University of Cologne 2003

[52] A. Krost, J. Christen, N. Oleynik, A. Dadgar, S. Deiter, J. Blásing, A. Krtschil, D. Forster, F. Bertram and A. Diez, Applied Physics Letters 85, 1496 (2004). 